University of Rhode Island

DigitalCommons@URI

Open Access Dissertations

1993

\title{
PHYSTCOCHEMICAL PROPERTIES AFFECTING THE PERCUTANEOUS ABSORPTION OF NIFEDIPINE
}

\author{
Emilio Squillante \\ University of Rhode Island
}

Follow this and additional works at: https://digitalcommons.uri.edu/oa_diss

\section{Recommended Citation}

Squillante, Emilio, "PHYSTCOCHEMICAL PROPERTIES AFFECTING THE PERCUTANEOUS ABSORPTION OF NIFEDIPINE" (1993). Open Access Dissertations. Paper 183.

https://digitalcommons.uri.edu/oa_diss/183

This Dissertation is brought to you for free and open access by DigitalCommons@URI. It has been accepted for inclusion in Open Access Dissertations by an authorized administrator of DigitalCommons@URI. For more information, please contact digitalcommons-group@uri.edu. 


\title{
PHYSICOCHEMICAL PROPERTIES AFFECTING \\ THE PERCUTANEOUS ABSORPTION OF NIFEDIPINE \\ BY \\ EMILIO SQUILLANTE
}

\begin{abstract}
A DISSERTATION SUBMITTED IN PARTIAL FULFILLMENT OF THE REQUIREMENTS FOR THE DEGREE OF DOCTOR OF PHILOSOPHY

IN

PHARMACEUTICS
\end{abstract}

UNIVERSITY OF RHODE ISLAND

1993 


\section{DOCTOR OF PHILOSOPHY DISSERTATION}

OF

EMILIO SQUILLANTE

APPROVED:

Dissertation Committee

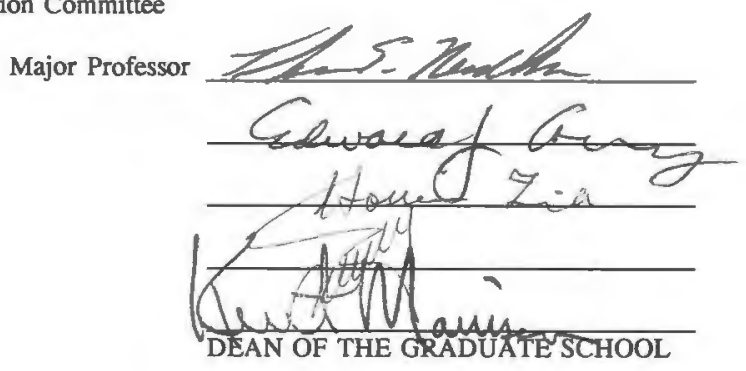

UNIVERSITY OF RHODE ISLAND 


\begin{abstract}
The effect of nifedipine saturation solubility in several cosolvent systems on nifedipine permeation through hairless mouse skin is evaluated. The effects of patch components is measured by (1) identifying those factors which significantly affect the permeation of nifedipine and (2) selecting settings for these factors which optimize flux and lag times. Of particular interest is the application of solubility theory toward the estimation of theoretical partition coefficients which were, it turn, useful in predicting the effect of donor composition on nifedipine permeation.

A stoichiometric approach was taken to examine the influence of permeation enhancers on skin permeability of this calcium channel blocker. Inherent in this approach is that a change in one component requires collateral change in the next; a deficiency best handled by an experimental design of the mixture type. The ultimate objective was to apply regression techniques to interpret, on a molar basis, the data obtained from in vitro permeation experiments. The model obtained was employed to: (1) identify both the magnitude and significance of synergism between formulation components and (2) optimize the desired response. Data from these inquiries have contributed toward the rational selection of components in a viable transdermal dosage form.
\end{abstract}




\section{ACKNOWLEDGEMENTS}

In preparing this work, 1 find myself indebted first and foremost to my major professor. I thank him for his many words and deeds on my behalf, particularly the brainstorming sessions that provided the wellspring of thoughts and ideas expressed in this thesis. The generosity of Lohmann Therapie Systeme, our German benefactors, is gratefully acknowledged. While at LTS I was the recipient of the boundless hospitality and insight of Dr. Walter Muller to whom I am especially grateful.

I acknowledge the patience and willingness of my committee and members of our department who have dealt with the unusual demands of this project, most notably the periods of sleep deprivation induced by the intense sampling schemes. Kudos go to Dr. Zia for first suggesting the use of dimethylisosorbide which helped permit the achievement of useful permeation rates. Charlene Yang is the Academic Computer Center's greatest natural resource. Pardeep Sethi imparted expertise on our initial skin permeation experiments and George Sienkevitch was helpful in the search for an appropriate experimental design to fulfill the special requirements of our approach. Their comments and criticisms were well received and much appreciated.

Finally, for those whose predilection has placed them on the path of graduate study, I close with some words of inspiration attributed to St. Francis of Assisi, "Start by doing what's necessary, then what's possible and suddenly you are doing the impossible." 


\section{PREFACE}

This body of work represents our observations, from conception to prototype, regarding the formulation of a poorly water soluble drug as a transdermal dosage form. Since the scope of the project was is unusually broad, we divide the main body of the dissertation into a general introduction followed by three chapters that address the issues of solubility, penetration enhancement and optimization.

The dissertation is best envisioned as proceeding chronologically through three distinct phases. Section I serves to introduce the interrelationship of several physical and chemical properties, particularly solubility phenomenon, on the permeation of a hydrophobic drug, nifedipine, through hairless mouse skin in vitro. Portions of this material have been presented at the 18th Symposium of the Controlled Release Society, Orlando, Florida, July 1992. A detailed investigation of representatives from several classes of penetration enhancers are presented. The final section documents the optimization of a proposed transdermal patch. Appendix A contains the source code used to process the data. Appendix B is a general discussion of the requirements of the statistical design of mixture problems. Appendix $\mathrm{C}$ contains important experimental data such as the screening procedures used to determine the applicability of various components of the proposed transdermal dosage form. Finally, Appendix D addresses suggestions for future work. 


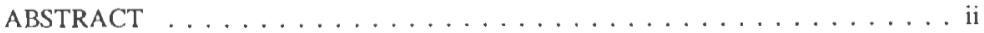

ACKNOWLEDGEMENTS $\ldots \ldots \ldots \ldots \ldots \ldots \ldots \ldots \ldots \ldots$

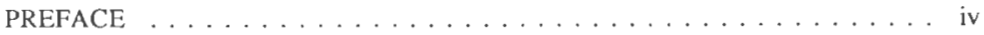

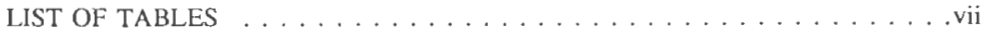

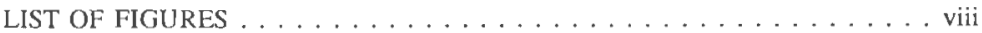

SECTION I. GENERAL INTRODUCTION

Rational for transdermal dosage forms .................. 1

Choice of Permeant . . . . . . . . . . . . . . . . . 2

Physicochemical properties of the permeant ............. 6

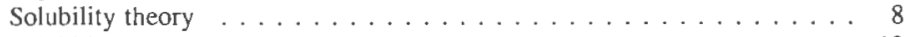

Solubility parameter . . . . . . . . . . . . . . . . . . . 12

Objectives of the Present Study .................... 24

\section{SECTION II. EXPERIMENTAL}

Transdermal delivery of nifedipine: feasibility studies based on solubility parameters ....................... 33

Application of solubility theory to component selection of a transdermal delivery system for nifedipine . . . . . . . . . . . 68

Formulation optimization . . . . . . . . . . . . . . . 94

\section{SECTION 11I. APPENDICES}

A. Computer program source code, description and listings . . . . . . . . 127

B. Numerical and statistical techniques .................. 143

C. Prototype transdermal device design . . . . . . . . . . . . . . 156

D. Conclusions and suggestions for future work . . . . . . . . . 166

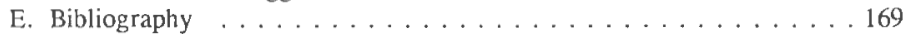




\section{LIST OF TABLES}

Table I. Physicochemical properties of various drugs delivered by the transdermal route of administration.

Table II. Effect of equilibration period on experimentally determined saturation solubilities of nifedipine in several solvents. . . . . . . . . 52

Table III. Solubility and diffusion from nifedipine suspended in morpholine derivatives. . . . . . . . . . . . . . . 55

Table IV. Saturation solubility in and permeability of nifedipine from binary mixtures prepared by the addition of propylene glycol or polyethylene glycol in dimethylisosorbide. . . . . . . . . . . . 58

Table V. Effects of alkyl chain length on skin permeation of nifedipine from binary mixtures containing alkanoic acids at the $10 \%$ level. . . . . 75

Table VI. Effects of alkyl chain length on skin permeability of nifedipine from suspensions of various alcohols at the $10 \%$ level. . . . . . . . . . 76

Table VII. Composition, solubility, and skin permeability for quaternary cosolvent systems prepared by blending PG, IP, OA, AZ, ET, and DM at the $25 \%$ mole fraction level. . . . . . . . . . . 77

Table VIII . Qualitative predictors of nifedipine permeation. . . . . . . . . 85

Table IX. Effect of drug level on permeation: comparison of suspension versus solution. ..................... 88

Table X. Design I. Quaternary systems data for a four-component simplex-centroid design: PG, OA, ET, and DM for donor suspensions using the following constraints: $P G=10-75, O A=10-36$, $\mathrm{ET}=10-36, \mathrm{DM}=10-75$.

Table XI. Design II. Four-component simplex-centroid blending propylene glycol $(\mathrm{PG})$, cis-oleic acid (OA), ethanol (ET), and

dimethylisosorbide (DM) for donor suspensions keeping [OA] and $[\mathrm{ET}] \leq 10 \%$.

Table XII. Quatemary systems data for a four-component blending propylene glycol (PG), cis-oleic acid (OA), ethanol (ET), and dimethylisosorbide (DM) donor suspensions keeping OA:ET ratio constrained at $10: 10 \ldots \ldots \ldots \ldots$. . . . . . . . . . . . . . . . . . . . . . . .

Table XIII. Parameter estimates from data presented in Table XI. . . . . . . . . . 110 Table XIV. Analysis of Variance of data presented in Table XI. . . . . . . . . 111 Table XV. Ridge analysis of data in Table XI . . . . . . . . . . . . . 112 Table XVI. Properties of acrylic, rubber-based and silicone matrix and pressure sensitive adhesives. . . . . . . . . . . . . . . 16 


\section{LIST OF FIGURES}

Figure 1. Estimation of nifedipine solubility parameter by Fedor's method. .......................... 43

Figure 2. Structure of nifedipine and its photodecomposition products. . . . . . 45

Figure 3. Ultraviolet-visible absorbance scan of nifedipine before (A) and after (B) 30 minute exposure to ambient light. . . . . . . . . . . . 47

Figure 4 . Saturation solubility $(\mathrm{mg} / \mathrm{ml})$ versus total solubility paraneter for the compounds studied. . . . . . . . . . . . . . . . 50

Figure 5. Nifedipine solubility in polyethylene glycol 400:dimethylisosorbide binary cosolvent mixtures. . . . . . . . . . . . . . 53

Figure 6. Nifedipine solubility in propylene glycol:dimethylisosorbide binary cosolvent mixtures. . . . . . . . . . . . . . . . . . 54 54

Figure 7. Nifedipine solubility in proplyene glycol, oleic acid, ethanol and dimethylisosorbide quatemary cosolvent mixtures. . . . . . . . . 56

Figure 8. Differential scanning calorimeter thermogram for nifedipine. . . . . . 57

Figure 9. Relationship between theoretical log skin-vehicle partition coefficient, amount permeated over 24 hours and solubility parameter for PEG:DMI binary mixtures. . . . . . . . . . . . . . 62

Figure 10. Relationship between theoretical log skin-vehicle partition coefficient, amount permeated over 24 hours and solubility parameter from PG:DMI binary mixtures. . . . . . . . . . . . . 63

Figure 11. Enhancement potential of binary mixtures of equimolar (10.6 $\mu \mathrm{mol}$ ) alkanoic acids in DMI. Also shown are the effect of addition of Azone at 1 and $10 \%$ mole fraction level.

Figure 13. Effect of cis-oleic acid on nifedipine permeation from dimethylisosorbide based donor formulations. . . . . . . . . . . . 81

Figure 14. Effect of $2 \%$ Azone on nifedipine permeation from IP:OA:ET:PG suspensions $(n=3) \ldots \ldots \ldots 3$

Figure 15. Optimization of PG:OA:ET:DM formulations based on the ridge analysis in Table XIV: Permeation of nifedipine from formulation P42.1 in intact and stripped skin. . . . . . . . . . . 86

Figure 16. Experimental sample space for the four component McLean Anderson mixture design $\left(X_{1}=P G ; X_{2}=O A ; X_{3}=E T ; X_{4}=D M\right) . \ldots 101$

Figure 17. Effect of high and low levels of dimethylisosorbide in quaternary mixtures holding ratio of OA:ET at $10: 10, n=4 . \ldots 106$

Figure 18. Effect of trebling amount of cosolvent applied from 6 to 18 microsnoles. . . . . . . . . . . . . . . . . . . 108

Figure 19. Three dimensional response surface and contour map of the predicted flux in quaternary mixtures studied (oleic acid and ethanol both at the $10 \%$ level).

Figure 20. Three dimensional response response surface and contour map of 
the predicted lag time in the quaternary mixtures studied (oleic acid and ethanol both at the $10 \%$ level). . . . . . . . . . . . 115

Figure 21. Contours of the predicted flux for polyhedron in Figure 16:

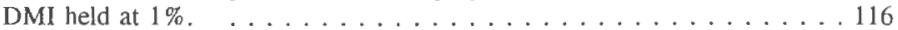

Figure 22. Contours of the predicted flux for polyhedron in Figure 16:

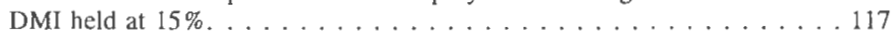

Figure 23. Contours of the predicted flux for polyhedron in Figure 16:

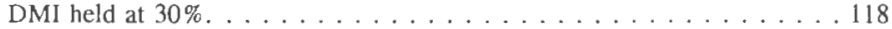

Figure 24. Contours of the predicted flux for polyhedron in Figure 16:

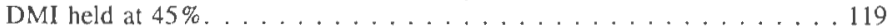

Figure 25 . Contours of the standard error of predicted flux responses

for formulations in Figure 23. . . . . . . . . . . . . . . . 120

Figure 26. Permeation of nifedipine from formulations of high

propylene glycol content. . . . . . . . . . . . . . . . 121

Figure 27. Schematic of prototype resevoir transdermal delivery

system. . . . . . . . . . . . . . . . . . 160

Figure 28. Nifedipine permeation from resevoir device or suspension

of OA:ET:PG $(7: 21: 72)$ applied in vitro $\ldots \ldots \ldots \ldots 3$ 


\section{SECTION I}

\section{GENERAL INTRODUCTION}

Rational for transdermal dosage forms - The potential of skin as a site of administration for systemically active drugs is evidenced by several commercially successful transdermal delivery systems (TDS). The drug delivery market was worth $\$ 8$ billion in 1988; of this transdermal systems constituted $\$ 324$ million in 1988 and is expected to reach $\$ 1$ billion by 1993 .' Since the early 1980 's, several innovative pharmaceutical products have demonstrated the merits of delivering therapeutic agents through the skin. ${ }^{2}$ Optimally designed transdermal dosage forms release drug in vivo according to pharmacokinetically rational rates so that concentrations are maintained within therapeutically desirable ranges. Drugs poorly available by the oral route may achieve therapeutic concentrations since drugs absorbed percutaneously are not subject to hepatic first pass metabolism. The transdermal route of administration may increase compliance by minimizing the inconvenience of remedication. This is a decided advantage in conditions e.g. hypertension where the effect of the disease is often sublime. The net result is a sustained, reliable, extended duration of drug action similar to an intravenous infusion without the many disincentives associated with the intravenous route of administration.

As the concepts underlying drug delivery system design becomes clearer ${ }^{3,4 .} 5 \& 6$ the potential of skin as a site of administration for systemically active drugs is being realized. Unfortunately, the transdermal route of administration cannot be employed 
for a large number of drugs and the problems that limit drug suitability have been outlined and discussed. ${ }^{7 \& 8}$ Briefly, the skin is an efficient barrier to the ingress of foreign materials so that few drugs penetrate the skin at rates sufficient to permit clinically useful transdermal application. Transdermal delivery of drugs for systemic effects is currently limited to nonirritating, lipophilic drugs of small molecular weight (less than 1000 daltons) and high potency (less than $21 \mathrm{mg}$ per day) that enter the skin by passive diffusion. Of the several layers of epidermis, the stratum corneum is generally recognized as the primary barrier to transdermal diffusion. The contribution of the stratum comeum to overall diffusional resistance tends to be larger for molecules of low molecular weight and lipophilicity. ${ }^{9}$ Formulations and methodologies which permit more than a few milligrams of drug to be delivered across this barrier would expand the range of candidate drugs that might be successfully marketed as a TDS. In the case of hydrophobic drugs it remains unclear how to determine a priori the optimal physicochemical properties required in a transdermal delivery system to best overcome the barrier function of the skin. Thus, research efforts targeted toward maximizing penetration have unquestionably high clinical and financial implications.

Choice of Permeant - With the exception of a recent publication by Diez et al ${ }^{10}$, no comparative percutaneous absorption studies have been done to evaluate the dihydropyridine calcium channel antagonists as suitable TDS candidates. Nifedipine,(dimethyl 1,4-dihydro-2,6-dimethyl -4 , (nitrophenyl)-3,5-pyridine dicarboxylate) is an ideal model permeant for this 
research. Nifedipine is an active calcium-channel antagonist that exerts its pharmacological action by blocking postexcitation of $\mathrm{Ca}^{++}$ions into cardiac and vascular smooth muscle. Since its discovery in 1968, nifedipine has become accepted as a first line treatment of angina and hypertension. ${ }^{11}{ }^{12}$ Nifedipine is poorly water soluble and its bioavailability is low when administered orally in the solid crystalline form. Previous pharmacokinetic studies have shown extraordinary interindividual variability of the plasma levels of the drug following oral administration. ${ }^{13}$ The variability in peak plasma levels (as much as 10 fold) is presumably due to the wide differences in oral bioavailability and first pass metabolism. The development of an alternative to the oral route that would bypass these significant, highly variable effects would offer significant advantages. Also, since transdermal administration minimizes pulse entry into the systemic circulation, undesirable side effects associated with unnecessarily high plasma levels of drug may be avoided.

The average steady state plasma concentration of nifedipine, following an average oral daily dose of $30 \mathrm{mg}$ per day, is $47( \pm 20) \mathrm{ng} / \mathrm{ml} .^{14}$ Clinical studies have shown that the hypotensive effect as well as the untoward side effects are correlated with the plasma nifedipine concentration. ${ }^{15}$ When the plasma concentration of nifedipine increases rapidly, there is a marked increase in heart rate and little effect on blood pressure. Conversely, this undesirable situation is reversed when administered by slow intravenous infusion or sustained release tablet. ${ }^{16}$ Transient, high plasma levels observed following oral administration of conventional capsules may vary by tenfold and increase the likelihood of undesirable high hemodynamic 
effects. ${ }^{17}$ An important feature of transdermal delivery systems is that they permit sustained, constant drug levels over a day or more. The transdermal route is also appealing since it can provide a zero-order rate of drug delivery, reduce problems associated with the aforementioned side effects and thereby improve patient compliance. The success of the Estraderm ${ }^{\mathrm{TM}}$ patch is evidence for the real need for the controlled delivery of drugs that undergo significant first pass effect. Transdermal estradiol relieves postmenopausal symptoms with one-tenth the amount of an oral dose of estrogen because the hormone enters the bloodstream directly, thus avoiding first-pass liver metabolism. ${ }^{18}$ Excessive estrogen levels (and side effects) are reduced when the drug is administered by a TDS. Nitroglycerin, the mainstay of anginal therapy, has enjoyed wide acceptance in patch form since once-a-day application contributes significantly towards patient compliance and avoids the large fluctuations in plasma levels following the oral administration. Finally, an important argument in favor of nifedipine is that the constant delivery of nifedipine is not thought to lead to the establishment of tolerance as seen with nitroglycerin. ${ }^{19}$

Following oral administration as a soft elastic gelatin capsule, nifedipine is completely absorbed with an absorption half life of 4 hours. Following intravenous administration, the drug is rapidly cleared from the plasma. When administered orally, nifedipine undergoes significant, variable first pass metabolism resulting in $\mathrm{C}^{\max }$ values between $40-200 \mathrm{ng} / \mathrm{ml}$ and an absolute bioavailability of $45 \%$ to $68 \%{ }^{20}$ Binding of nifedipine to plasma protein is concentration dependent and ranges from 92-98\%. The drug appears to distribute in a volume slightly larger than total body 
water. The elimination half life of 2 to 3 hours necessitates administration at intervals of every four to six hours.

Convenient once a day dosage regimens are highly desirable in general, and especially for the treatment of asymptomatic diseases such as hypertension.

Shortcomings associated with its rapid elimination following oral administration prompted the development of innovative drug delivery systems capable of zero-order drug delivery that prolong pharmacologic activity over a 24 hour period. ${ }^{21} 22$ The Pfizer Procardia XL ${ }^{T M}$ tablet utilizes OROS $^{\text {TM }}$ technology to deliver the daily nifedipine requirement over a 24 hour period. ${ }^{23}$ However, the bioavailability of the Pfizer extended-release tablet is reduced by $55-65 \%$ of that achieved with the same doses administered by conventional soft gelatin capsules. Following multiple doses, oral bioavailability from the extended-release tablet is approximately $75-86 \%$ of that achieved with the same doses administered by conventional capsules. ${ }^{24}$ Thus, more than twice the clinically useful amount of nifedipine is unnecessarily ingested on a chronic basis in order to maintain plasma levels in the therapeutic range.

The theoretical transdermal daily dose equivalent to the minimum oral daily dose of $30 \mathrm{mg}$, adjusted for a bioavailability factor of 0.6 , is $13 \mathrm{mg}$. The results reported by Diez, et al. permit only $1 \mathrm{mg}$ of nifedipine to be delivered transdermally over 24 hours under steady state conditions. When considered in light of an average nifedipine elimination clearance of 83 liters/hour, their optimum flux values translate to a predicted steady state plasma level of only $0.53 \mathrm{ng} / \mathrm{ml}$, far short of the target therapeutic plasma concentration of $6-51 \mathrm{ng} / \mathrm{ml}$. 
Physicochemical properties of the permeant - Knowledge of the permeant's molecular structure, physicochemical properties, physicochemical compatibility and stability profiles are the initial key considerations in selecting a probe. Several guidelines which correlate the physicochemical properties of drugs with their ease of skin penetration have become apparent. ${ }^{25,26, \& 27}$ In general, lipid-like molecules are more easily delivered through the skin than are hydrophilic ones; polar substances are not as well absorbed as nonpolar ones; and organic liquids are better absorbed than solids. For the purpose of comparison, Table I lists some of the salient physicochemical properties of various drugs that have been successfully delivered by the transdermal route of administration. Molecules like nifedipine which have proportionately higher lipid solubility, low melting point and low molecular weight should permeate the skin at greater rates. Nifedipine's molecular weight is similar to that of fentanyl, another drug commercially available as a transdermal dosage form. Nifedipine, like estradiol, has a relatively high melting point. All three drugs are classified as "extremely lipophilic", as demonstrated by their n-octanol:water partition coefficient. Of the drugs listed, the steroids bear the best overall similarity and strategies used to enhance their penetration theoretically should apply equally well to nifedipine.

Nifedipine is compatible with many pharmaceutically useful solvents, thermostable, and nonhygroscopic. Owing to the extreme low basicity of the dihydropyridine nitrogen it is not possible to obtain stable salts with acids. 
Table I. Physicochemical properties of various drugs delivered by the transdermal route of administration.

\begin{tabular}{||l|c|c|c|c|c||}
\hline \multicolumn{1}{|c|}{ DRUG } & $\mathrm{M} . \mathrm{W}$. & $\mathrm{T}_{\mathrm{m}}$ & $\Delta \mathrm{H}_{\mathrm{f}}$ & $\mathrm{C}_{\mathrm{s}}$ & $\log [\mathrm{P}]^{\mathrm{a}}$ \\
\hline estradiol & 272.4 & 176 & $* * *$ & 0.002 & $3.9(2.3)$ \\
\hline fentanyl & 336.5 & 83 & $* * *$ & $0.01-75$ & 2.9 \\
\hline hydrocortisone & 363.5 & 218 & 8.1 & 0.389 & $1.6(-0.2)$ \\
\hline metronidazole & 171.2 & 159 & $* * *$ & 8.5 & 0.85 \\
\hline nicotine & 162.2 & $1 \mathrm{q}$ & 9.4 & $10-50$ & 1.17 \\
\hline nifedipine & 346.3 & 173 & 9.09 & 0.006 & 3.1 \\
\hline progesterone & 314.5 & 121 & $* * *$ & 0.009 & 3.9 \\
\hline salicylic acid & 138.1 & 157 & 141 & 2.2 & $1.8(1.2)$ \\
\hline testosterone & 288.4 & 155 & 6.2 & 0.023 & $3.9(3.3)$ \\
\hline theophylline & 180.2 & 270 & 7.1 & 8.3 & $-0.7(-2.1)$ \\
\hline \hline
\end{tabular}

Key: $M . W .=$ molecular weight in daltons, $T_{m}=$ melting point in ${ }^{\circ} \mathrm{C}, \Delta \mathrm{H}_{\mathrm{r}}$ in kilocalories per mole, $\mathrm{C}_{s}$ in milligrams per mililiter, ${ }^{\mathrm{a}} \log [\mathrm{P}]=$ octanol/water partition from Hansch and Leo, 1979 (in parentheses are isopropyl myristate/water partition coefficients from Surber et $\mathrm{al}^{28}$ ). References unless otherwise noted: estradiol $^{29}$, fentanyl ${ }^{303132}$, hydrocortisone $e^{33}$, metronidazol $e^{34}$, nicotine ${ }^{3536}$, progesterone $^{37}$, salicylic acid, testosterone ${ }^{38}$, theophylline ${ }^{39}$. 
Since the rate of nifedipine absorption from the gastrointestinal tract is independent of $\mathrm{pH}$ over the range of 1.2-7.5 the effect of $\mathrm{pH}$ on skin permeation would probably be negligible. Nifedipine is thermostable and nonhygroscopic. Methanolic solutions stored at room temperature for 6 months have displayed no detectable decomposition. There is no influence of $\mathrm{pH}(2-12)$ and ionic strength on stability. It is extremely sensitive to light below $450 \mathrm{~nm}$. The major metabolite, a nitropyridine, formed by enzymatic oxidation is identical to that formed by photodegradation under visible light. High Pressure Liquid Chromatography (HPLC) provided a straightforward, validated, stability-indicating analytical method of quantitatively measuring nifedipine and its primary breakdown products in vitro ${ }^{40}$ and in vivo. ${ }^{41}{ }^{42}$

In summary, having considered the physical characteristics of the drug and proposed TDS, it appears that the physical, chemical and pharmacokinetic attributes of nifedipine conform with those required of a drug candidate for transdermal delivery system development.

Solubility theory - The solubility of a drug in one or more components of a pharmaceutical dosage form is valuable information which can be used to characterize its behavior. This is especially true of topical dosage forms where the solubility of drug can be related to dissolution rate, thermodynamic activity, vehicle release rate, and apparent permeation rates. Transdermal delivery is a diffusion process driven by the establishment of a thermodynamic activity gradient of diffusant within the skin. When a drug is applied topically, it diffuses passively out of its carrier vehicle into the surface tissue of the skin. The movement continues through the stratum corneum 
and into the viable epidermis and dermis with the gradient terminating at the upper reaches of the microcirculation of the skin in the dermal layer. The fundamental equation describing diffusion in membranes such as skin is Fick's First Law. For the steady state case, the amount $\mathrm{M}$ of material flowing through a unit cross section, $\mathrm{S}$, of a barrier in unit time, $t$, is known as the flux, $\mathrm{J}$,

$$
J=\frac{d M}{S * d t}
$$

The flux in turn is proportional to the concentration gradient, $d \mathrm{C} / d \mathrm{x}$ :

$$
J=-D \frac{d C}{d x}
$$

in which $\mathrm{D}$ is the diffusion coefficient of a penetrant, $\mathrm{C}$ is its concentration, and $\mathrm{x}$ is the distance of movement perpendicular to the barrier. When donor and receptor compartments are separated by a semipermeable membrane of cross-sectional area $S$ and thickness $h$, and the concentrations of the diffusant in the membrane in the donor and receptor sides are $C_{1}$ and $C_{2}$, respectively, then equation (1) may be rewritten

$$
\frac{d M}{d t}=D\left(\frac{C_{1}-C_{2}}{h}\right)
$$

The values of $C_{1}$ and $C_{2}$ within the membrane are difficult to determine experimentally but can be approximated by the partition coefficient, $\mathrm{K}$, multiplied by the concentration $\mathrm{C}_{\mathrm{d}}$ on the donor side or $\mathrm{C}_{\mathrm{r}}$ on the receiver side of the membrane 


$$
J=\frac{d M}{d t}=\operatorname{DSK}\left(\frac{C_{d}-C_{p}}{h}\right)=P S C_{d}
$$

for which, under sink conditions, $\mathrm{C}_{\mathrm{r}} \approx 0$. Thus, for a fixed surface area, the diffusion of permeant from donor to receptor is proportional to a gradient in thermodynamic activity. If the vehicle in no way changes the properties of the membrane (i.e., $\mathrm{D}$ and $\mathrm{K}$ remain constant) nor does the vehicle codiffuse with the drug then $\mathrm{P}=\mathrm{DK} / \mathrm{h}$ is a constant and equation (4) implies that an increase in the thermodynamic activity of a species in solution should be accompanied by an increase in permeability. While the activity of the permeant will vary according to the composition of solvent(s) used in the donor medium, it may be held constant for the duration of an experiment for a given donor by using a saturated solution in the presence of excess solid drug.

Many have recognized that intrinsic solvent effects significantly affect drug transport across the stratum corneum and underlying epidermal membranes ${ }^{43}$ and that variation in vehicle composition often has a marked effect on the release of drug from the dosage form. ${ }^{44}$ However, for those cases in which $\mathrm{P}=\mathrm{DK} / \mathrm{h}$ is a constant, enhancement of transdermal delivery by maximizing thermodynamic potential has been achieved with volatile and nonvolatile mixtures to enhance "the escaping tendency" of drug from a formulation by creating supersaturated conditions. ${ }^{45}$ If thermodynamic activity of a species in solution is increased, diffusivity should increase and equilibrium saturation solubility (which is a reflection of activity) should provide a measure of permeability. Accordingly, if the rate of delivery is directly 
dependent on the amount of drug in the donor then vehicle composition should have significant impact on the flux of drug through the skin by virtue of its inherent ability to solubilize the drug. Twist and Zatz observed the diffusion of parabens through silicone from a spectrum of polyol-water cosolvents and concluded that permeation is a function of permeant activity. ${ }^{46}$

Cosolvent mixtures can be combined to yield vehicles of variable polarity and are routinely used as aids to solubilize drugs in aqueous vehicles. Manipulation of solubility viz judicious choice of excipients has contributed to the successful marketing of two transdermal products. Isopropyl palmitate and isopropyl myristate are used as cosolvents to enhance the solubility of nitroglycerin in the polymer matrix type transdermal systems. The principle use of ethanol in the Estraderm patch is to enhance the solubility of 17-B-estradiol in the reservoir compartment of the device. The Duragesic patch also incorporates ethanol. Selection of the appropriate cosolvent system can maximize the activity, hence its flux yet minimize the potential for precipitation which may result following application. In practice it is difficult to distinguish whether flux enhancement is due to the vehicle effect on the skin to permeant solubility or a combination of the two. Nevertheless, prediction of solubility may be a useful initial tool in the design of TDS.

In general, a compound dissolves far easier in a solvent possessing related functional groups than in one of a completely different nature ${ }^{47}$. Stated another way, the best solvent for a particular solute is the one that most closely matches its polarity. While various qualitative generalizations based on molecular structure are 
available, a quantitative method would be more useful. $\mathrm{Kier}^{48}$ has proposed a numerical index that ranks solvents according to their polarity that can be quickly calculated, does not depend on the availability of the actual molecule, and permits prediction of the polarity of solvent or cosolvent mixtures. In the method of coincidence, the solubility of the solute in several solvents is plotted as a function of either dielectric constant or solubility parameter of solvent, whereupon the value in question is assumed to be equal to the corresponding value of the solvents in which it has the greatest solubility. The latter method is well accepted and has been employed to evaluate the solubility parameter of nifedipine.

Solubility parameter - The solubility parameter of solute $\left(\delta_{2}\right)$ and solvent $\left(\delta_{1}\right)$ was proposed by Hildebrand in an attempt to quantitate the effect of solute-solvent interactions on the solubility of solutes in solvents. It is defined as the square root of the cohesive energy density (CED), a fundamental thermodynamic parameter that allows one to estimate several thermodynamic properties of solutions. ${ }^{49}$ The CED is the net work expended by the removal of a solute molecule from the solid plus that expended through the opening of a 'hole' in the solvent minus that realized from the insertion of the solute molecule into this hole. The cohesive energy density itself is defined as the ratio of the energy of vaporization to the molar volume, the latter of which is often difficult to verify experimentally. Unlike other measures of polarity, the solubility parameter describes the physical size and strength of the intermolecular attractive fields. Therefore, they lend themselves to predictable mechanical explanations of intermolecular phenomena. In addition to its importance in the theory 
of solutions, the solubility parameter has been shown to be connected to other physical properties such as surface tension, wettability, boiling points, glass transition temperatures of polymers, the strength of materials and is used to estimate the miscibility of multi-component mixtures. Thus the ability to estimate the solubility parameter can often be an extremely useful tool that is applicable to a diverse number of practical problems. According to the Hildebrand solubility parameter approach, two liquids are miscible if their solubility parameters differ by no more than 3-4 units. Remarkable combinations of non-solvents have been produced which have poor solvent strength individually while in combination exhibit good solvent strength. This synergism is displayed by the classic ethyl ether:ethanol $(\delta=7.4: 12.6)$ solvent combination used to solubilize nitrocellulose $(\delta=11.3)$. The robust nature of this approach lends itself to the problem of predicting permeant activity in the donor. Consequently, our efforts have been directed towards using the solubility parameter to predict percutaneous flux of nifedipine.

We employ the Hildebrand approach ${ }^{50}$ to obtain the logarithm of the ideal mole

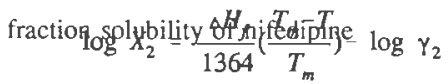

$$
\log \gamma_{2}=\frac{V_{2} \Phi_{1}^{2}\left(\delta_{1}-\delta_{2}\right)^{2}}{2.3 R T}
$$


where $X_{2}$ is the mole fraction solubility of nifedipine at temperature $T, \Delta H_{f}$ is the heat of fusion of the solute at its melting point $T_{m}$, and $\gamma_{2}$ is the activity coefficient of the drug in the vehicle. The first part of the right-hand side of equation (5) gives the ideal solubility, whereas the second part, $\log \gamma_{2}$, gives the contribution of the solutesolvent interactions to the deviations of the measured solubility, $X_{2}$, from the ideal solubility. Regular solution theory describes the activity coefficient of the drug in the vehicle, given by equation (6), where $\delta_{2}$ is the solubility parameter of $\mathrm{N}, \delta_{1}$ is the solubility parameter of the vehicle, $\mathrm{V}_{2}$ is the molar volume of $\mathrm{N}, \mathrm{R}$ is the gas constant and $T$ is absolute temperature. The volume fraction of the solvent, $\Phi_{1}$, is calculated from $^{51}$

$$
\phi_{1}=\frac{V_{1}\left(1-X_{2}\right)}{V_{1}\left(1-X_{2}\right)+V_{2} X_{2}}
$$

The main limitation has been that the solubility parameter concept is strictly applicable only to nonpolar systems where dispersion forces predominate. Other forces exerted by intermolecular interactions in liquids with similar solubility parameter may result in very different solubility profiles. This is due, in part, to differences in the three intermolecular forces commonly referred to as the London, Keesom and Hydrogen bonding attractions. Hanson and Beerblower ${ }^{52}$ partitioned the cohesive energies into three terms: $\delta_{d}$ is the dispersion forces observed in nonpolar, hydrocarbon mixtures, $\delta_{o}$ reflects dipole-dipole interactions or 'orientation' forces between molecules e.g., acetone, and $\delta_{h}$ describes the acid-base character associated with all varieties of strong donor-acceptor interaction between solute and solvent. 
This third term is not restricted to hydrogen bonding. The total cohesive energy density, $\Delta \mathrm{E} / \mathrm{V}$, for a species may be partitioned into contributions from dispersion forces, dipolar interactions, and hydrogen bonding:

$$
\begin{aligned}
& \frac{\Delta E}{V}=\frac{\Delta E_{d}}{V}+\frac{\Delta E_{\rho}}{V}+\frac{\Delta E_{h}}{V} \\
& \delta=\sqrt{\frac{\Delta E^{\nu}}{V}} \approx \sqrt{\frac{\Delta H^{\nu}-R T}{V}}
\end{aligned}
$$

$\Delta \mathrm{E}^{v}$ is the energy of vaporization of a liquid, $\Delta \mathrm{H}^{v}$ its enthalpy of vaporization, $R$ the gas constant, $\mathrm{T}$ is the absolute temperature, and $\mathrm{V}$ is the liquid molar volume. The quantity $\delta$ is the total solubility parameter and $\delta^{2}$ is the cohesive energy density for a solvent or solute allowing equation (8) to be rewritten as

$$
\delta^{2}=\delta_{d}^{2}+\delta_{p}^{2}+\delta_{h}^{2}
$$

The dispersion parameter, $\delta_{\mathrm{d}}$, is obtained from data for the compound's homomorph, defined as a saturated hydrocarbon having essentially the same chemical structure, size, and shape as those of the polar compound. The polar parameter, $\delta_{\mathrm{p}}$, was calculated using a modified equation from Bottcher:

$$
\delta_{p}^{2}=\frac{12108}{V^{2}} \frac{\epsilon-1}{\epsilon+n_{D}^{2}}\left(n_{D}^{2}+2\right) \mu^{2}
$$




$$
\delta_{p}^{2}=\frac{12108}{V^{2}} \frac{\epsilon-1}{\epsilon+n_{D}^{2}}\left(n_{D}^{2}+2\right) \mu^{2}
$$

The hydrogen bond parameter for a hydroxyl compound is obtained from

$$
\delta_{h}^{2}=\sqrt{\frac{\Delta H}{V}}
$$

where $\Delta \mathbf{H}$ is an average enthalpy of formation for hydrogen bonding, $4650 \mathrm{cal} / \mathrm{mole}$, for each hydroxy group. The partial solubility parameters of Hansen are obtained from the literature ${ }^{53}$ or estimated using one of several group contribution methods. ${ }^{54}$ Solubility and partition coefficient are two factors of crucial importance to consider in the design and development of new dosage forms. While readily estimated, ${ }^{5,5}$ it is sometimes unfeasible or impossible to physically measure either property in some systems. The theoretical partition coefficient and ideal mole fraction solubility can be estimated using solubility parameters. Fortunately, the solubility parameter itself is easily and accurately estimated from a knowledge of molecular composition. Methods that permit the estimation of a compound's solubility parameter based on functional group contributions are based on the assumption that group increments exist which may be summed over the known structure of the substance. A BASIC program for the calculation of solubility parameters of compounds of pharmaceutical interest has been reported. ${ }^{56}$ Values obtained are in good agreement with values determined experimentally. These results are noteworthy, given that when Hildebrand established the thermodynamic principles of intermolecular cohesion, it was restricted to nonpolar solutes in nonpolar solvents. 
Of the various methods proposed for estimating the solubility parameter, we have chosen the method proposed by Fedors ${ }^{57}$ for two reasons: (1) the contribution of a much larger number of functional groups were evaluated and (2) the method requires only a knowledge of the structural formula of the compound.

The distribution of a drug between two phases, represented by the term $\mathrm{K}$ introduced in equation (4), is given in equation (13)

$$
\log K=\log \frac{\gamma_{2}}{\gamma_{\text {skin }}}
$$

Since each activity coefficient in equation (13) can be calculated from equation (6), a theoretical partition coefficient can be calculated by substituting the values for the calculated activity coefficients into equation (13). Use of the difference between the solubility parameter of drug and membrane to explain differences in biological activity of drugs was first introduced by Mullins. ${ }^{58}$ Sloan used the expanded form of equation (13) as a means of calculating theoretical solubilities of theophylline in hairless mouse skin so that theoretical vehicle-membrane partition coefficients between skin and various polar and nonpolar vehicles could be estimated..$^{59}$ In a separate study, salicylic acid was used as a model compound to study the relationship between the solubility parameter of drug and membrane on transport through lipoidal membranes. ${ }^{60}$ In both cases, a good fit between the theoretical partition coefficients and the experimentally determined permeability coefficients were obtained. The influence of solute solubility on flux in vivo has also been noted for estradiol. ${ }^{\circ 1}$ We propose to examine the properties that seem to limit drug suitability so that the 
rational formulation of a new generation of transdermal systems can occur.

Penetration enhancers - Of the various layers of the skin, the stratum corneum is believed to pose the greatest diffusional resistance. Since diffusion through the stratum corneum is usually the rate limiting step, enhancement of permeability is usually directed toward reversibly altering the properties of the stratum corneum. The term "penetration enhancer" is applied to agents which reduce the diffusional resistance of the stratum corneum, by reversibly damaging it, or by altering its physicochemical nature. The previous discussion considers only the simple situation in which diffusion occurs in a single homogenous medium. However, skin is a heterogeneous multilayer tissue. The concentration gradient developed over several strata is analogous to an electrical circuit, either in series or in parallel, where each strata contributes a unique diffusional resistance. While the total resistance of the composite is essentially a function of the high resistance of the stratum corneum, it is incorrect to attribute the properties of one single layer to the individual properties of the composite layers. Modeling the skin in this superficial manner may give rise to false assumptions on how these properties may be altered. That the permeation process is more complex than the simple case of diffusion through the stratum comeum suggests and that the subdivisions of the skin should be considered individually.

The partition coefficient is crucially important in promoting a high initial concentration of the agent within the upper strata of the epidermis. Nonpolar 
molecules are expected to diffuse passively though the lipid domains of the stratum corneum intercellular space. If the intercellular transepidermal pathway transport mechanisms control drug passage through skin, one would expect the partioning behavior of the drug to influence its permeation and a correlation should exist between partition and permeability that, in turn, relates structure to permeant flux. In practice, partition coefficients based on various reference systems are often unreliable predictors of relative permeabilities but at least constitute a frame of reference from which to envision drug movement through the skin. Given the high octanol-water partition coefficient of nifedipine $(10,000: 1)$ and that the solubility parameters of porcine skin and nifedipine are nearly identical leads to the supposition that: (1) nifedipine should have high affinity for and readily penetrate stratum corneum lipids and (2) significant resistance to nifedipine diffusion should reside in the deeper dermal layers. Scheuplein had calculated that a tissue-water partition coefficient of $>400$ was required before a 200 micron layer of dermis had a diffusional resistance comparable to that of stratum corneum 10 microns thick. ${ }^{62}$ Indeed, our experiments on stripped skin have confirmed that a significant barrier to diffusion still exists even when the stratum corneum has been removed. Therefore, the ideal patch design would encompass the following functions: (1) initial high activity of drug in the patch creates a severe gradient forcing the drug from the patch into the epidermis; (2) release of enhancer(s) to alter the diffusion coefficient of the drug; and (3) release of cosolvent at a prescribed rate so as to modify nifedipine solubility in the lower epidermal and dermal layers. 
Chemical enhancers can be grouped into two general categories: amphoteric molecules (such as Azone and oleic acid) and cosolvent molecules (such as dimethylsulfoxide, propylene glycol, and ethanol). ${ }^{63}$ Amphoterics are thought to enhance permeation by increasing the diffusivity of the permeant through the stratum corneum, while cosolvents are used to promote the partitioning of drug in the skin layers. It is often difficult to distinguish from the array of possible mechanisms by which an ingredient may influence permeant diffusion. For example, surfactants can alter the kinetics of drug permeation through the skin by a direct effect on the skin barrier, through complexation or solubilization of the drug, by improving wetting of the membrane or through alteration of the permeability of skin to water. ${ }^{64} 6566$

Generally, a penetration enhancer is selected based on the physicochemical properties of the permeant. Since lipophilic drugs are believed to penetrate the stratum corneum by passive diffusion though the intercellular lipids, materials (e.g., oleic acid or Azone) that are known to increase the diffusivity of permeants in the 'lipoidal' pathway should be better suited than enhancers that affect the proposed 'polar' pathway. Alternatively, the polar pathway, proposed to account for the anomalous permeation of small or ionizable molecules, would seem unsuited for nifedipine, which is a molecule of moderate molecular weight, insignificant pKa, and poor water solubility. A preliminary saturation solubility profile compiled from the literature shows nifedipine to have affinity for solvents of moderate dipole moment with minimal hydrogen bonding interactions. Nifedipine appears to lie midway in the lipophilic-hydrophilic continuum, prohibiting generalizations regarding passage via a 
lipoidal or polar route and, of course, the subsequent choice of enhancer. For permeants with poor solubility profiles, the cosolvent-type permeation enhancers have been successfully employed either alone or in combination. Generally, these enhancers exert their influence rapidly, but this influence diminishes relatively soon after the delivery system has been removed from the skin. Because they penetrate the skin, there may be questions regarding toxicity and irritation during chronic application.

Utilization of enhancers to optimize transdermal delivery introduces potential adverse interactions between drug, enhancer, system components, and skin. ${ }^{67}$ Unfortunately, it remains unclear how to best choose ideal enhancer combinations. An interesting approach to enhancer selection is based on the use of quantitative structure-activity relationships to predict the pharmacological properties of organic compounds. This is accomplished by the assignment of organic and inorganic values for a given compound depending on structural components. ${ }^{68}$ Hori obtained conceptional diagrams on which classical enhancers and newer enhancers were located in quite different regions. What emerged was a clear relationship between pharmacological actions and the physicochemical properties of organic compounds. These studies provided an impetus for our search for appropriate enhancers, based on physicochemical properties, that interact in a positive synergistic manner to enhance nifedipine delivery. A review of materials considered in the present work now follows.

Alcohols - Studies using ethanol ${ }^{p 9}$ and the other lower n-alkanols ${ }^{70}$ on hairless mouse 
stratum comeum have provided no evidence of direct effects on lipid alkyl chain packing, mobility, and conformational order. Knutson et al ${ }^{71}$ suggested that ethanol functions as an effective "fluidizer" in the stratum corneum lipid bilayer on some locus at or near the polar head plane rather than the bilayer hydrocarbon interiors. Ethanol is presumed to exert its influence by swelling and softening the stratum corneum, thereby reducing its effectiveness as a permeation barrier.

A reservoir-type therapeutic transdermal system using pure ethanol or ethyl acetate to provide constant release of estradiol are well tolerated. ${ }^{72}$ The effects of ethanol on the adhesive strength of a variety of control membrane laminates have been examined. ${ }^{73}$ Alza controls a patent on ethanol as an absorption enhancer and it is incorporated in their Estraderm and Duragesic products. Estradiol alone cannot permeate the skin to produce adequate plasma levels, hence the Estraderm patch uses ethanol to promote the partitioning of the drug into the stratum corneum and so reduce this barrier's resistance to drug transport. The transport rate from a saturated ethanol/water donor suspension is approximately 20 times that found for aqueous suspensions. In the Duragesic patch, alcohol $\left(0.1 \mathrm{ml} / 10 \mathrm{~cm}^{2}\right)$ is used to increase the permeability of the skin to fentanyl and to enhance the rate of drug flow through the rate-limiting membrane. Only a small amount of alcohol $\left(<0.1 \mathrm{ml}\right.$ per $\left.10 \mathrm{~cm}^{2}\right)$ is actually released from the system during a 72-hour application period. A linear dependence of ethanol on drug penetration has been observed in nitroglycerin ${ }^{74}$ and for the ethanol-ethyl-acetate cosolvent in levonorgestrel. ${ }^{75}$

Twist and Zatz have shown that the relative flux from a series of alcohols is 
correlated with the amount of alcohol imbibed by polydimethylsiloxane membranes. ${ }^{76}$ Good et $\mathrm{al}^{\pi}$ reasoned that because there was a good correlation between the flux of ethanol and estradiol, an important feature of an estradiol TDS is to control the rate of ethanol release which in turn would moderate the flux of the drug through the skin. Smith, et al, ${ }^{78}$ measured plasma estradiol levels following application of Estraderm patches with and without additional amounts of ethanol added over a period of one week. Addition of $0.6 \mathrm{ml}$ of ethanol on day 3 significantly extended the mean time 0(by 50 hours) for serum estradiol levels to return to baseine without increasing peak absorption. These observations are noteworthy: ethanol, a polar molecule, has enhancing effects on lipophilic molecules which are transported mainly through the lipoidal pathway and this effect is somehow linked to the rate and extent of alcohol uptake into the membrane.

Propylene Glycol (PG) - PG has been shown to significantly increase the solubility of drugs in the stratum corneum and therefore enhance the permeability of the skin. ${ }^{79}$ Cited as a penetration enhancer, PG also alters the release rate of steroids by its effect on permeant thermodynamic activity. This may be partially explained by the ability of $\mathrm{PG}$ to permeate the skin in substantial amounts. ${ }^{80,81}$ PG may both increase and decrease permeation of substances and appears to aid penetration best when combined with surface active agents. ${ }^{82}$ Nomura et al. ${ }^{83}$ observed that PG was readily absorbed through rat skin and that $\mathrm{PG}$ percutaneous absorption profiles were similar to those of indomethacin, suggesting that $\mathrm{PG}$ and indomethacin penetrate together through the skin. Sheth et $\mathrm{al}^{84}$ reported the influence of Azone, propylene glycol and polyethylene 
glycol on in vitro skin penetration of triflurothymidine. The synergistic advantage of multipie enhancers on estradiol permeation has been displayed by the combination of Azone with propylene glycol. ${ }^{85}$

Fatty acid \& alcohols - Palmitic, oleic and linoleic acids are the major free fatty acids present in serum and comprise 27,25 and $13 \%$, respectively, of total circulating, non-esterified fatty acids. ${ }^{86}$ Long-chain fatty acids have been shown to be effective penetration enhancers for several lipophilic molecules through hairless mouse skin in the presence of cosolvents. ${ }^{87}$ Work performed in a multicenter collaboration established that the cis-isomer of oleic acid disrupts the "lipid fluidity" of the stratum corneum; the trans isomer did not demonstrate this effect. ${ }^{88}$ This increased chain mobility is the hypothetical 'fluidization' of lipid bilayers thought to be responsible for the penetration enhancement of agents that act on the lipoidal pathway such as oleic acid ${ }^{89}$ In light of the considerable attention with regard to their effect on membrane permeability, the effect of long chain fatty acids and alcohols on nifedipine permeation was investigated.

\section{Objectives of the Present Study}

1. Screening studies targeted toward the identification of formulation components that enable the model permeant, nifedipine, to be delivered in measurable quantities across a semipermeable membrane.

2. Extension of our knowledge regarding the influence of a permeant's 
physicochemical attributes on patch design and performance with the intent that such inquires may serve as a paradigm in the development of new transdermal delivery systems.

3. Assess the value of using mole fraction comparisons between patch components using statistical designs developed for problems involving mixtures.

4. Material selection process and compatibility studies for fabrication of a transdermal delivery system prototype. 


\section{REFERENCES}

1. Colombo, Peter. "World News." Controlled Release Newsletter 1990, 817.

2. Brown, Leonard and Robert Langer. "Transdermal Delivery of Drugs." Ann. Rev. Med. 1988, 39, 221-229.

3. Kydonieus, A.F. "Fundamentals of Drug Delivery" in Transdermal Drug Delivery. Vol. 1, A.F. Kydonieus and B. Berner, Eds. Boca Raton, Florida: CRC Press, 1990.

4. Wiechers, J.W. "The Barrier Function of the Skin In Relation to Percutaneous Absorption of Drugs." Pharmaceutisch Weekblad-Scientific Edition 1989, 11:6, 185198.

5. Barry, W. "Modern Methods of Promoting Drug Absorption Through the Skin." Molecular Aspects of Medicine 1991, 12, 195-241.

6. Loth, H. "Skin Permeability." Methods and Findings in Experimental and Clinical Pharmacology 1989, 11, 155-164.

7. Guy, Richard H. and Jonathan Hadgraft. "Selection of Drug Candidates for Transdermal Drug Delivery." in Transdermal Delivery Systems: Developmental Issues and Research Initiatives New York, N.Y.: Marcel Dekker, 1989. 59-82.

8. Yum S.I. "Transdermal Therapeutic Systems and Rate Controlled Drug Delivery." Medical Program Technology 1989, 15, 47-52.

9. Izumoto, T., A. Aioi, S. Uenoyama, K. Kuriyama, and M. Auma. "Relationship Between the Transference of a Drug from a Transdermal Patch and the Physicochemical Properties." Chem. Pharm. Bull. 1992, 40, 456-458.

10. Diez, I., H. Colom, J. Moreno, R. Obach, C. Peraire, and J. Domenech. "A Comparative In Vitro Study of Transdermal Absorption of a Series of Calcium. Channel Antagonists." J. Pharm. Sci. 1991, 80, 931-936.

11. Hansson, L. "Calcium Antagonists: An Overview." Am. Heart Jour. 1991, 122:1, 308-11.

12. Dollery, C.T. "Clinical Pharmacology of Calcium Antagonists." American J. of Hypertension 1991, 4, 888-958.

13. Duhm, Von B.; Maul, W.; Medenmwal, H.; Patzschke, K.; Wagner, L.A. "Tier Experimentelle Untersuchenger zur Pharmakokinetik und Biotransformation von Radioactiv Markiertem 4-(2'Nitrophenyl)-2,6-dimethy-1,4-dihydropyridin-3,5dicarbonsauredimethyl ester." Arnz. Forsch. 1972, 22, 42-46. 
14. Crotin, C.M. "Nifedipine Pharmacokinetics." Drug Int. Clin. Pharm. 1987, 21, 642-4.

15. Morimoto, K.; Tabata, H.; Morisaka, K. "Nasal Absorption of Nifedipine from Gel Preparations in Rats." Chem. Pharm. Bull. 1987, 38, 3041-3044.

16. Kleinbloesem, C.H., P. van Brummelen, and D.D. Breimer. "Nifedipine. Relationship Between Pharmacokinetics and Pharmacodynamics." Clinical Pharmacokinetics 1987, 12:1, 12-29.

17. Pasanisi F.; Merideth, P.A.; Reid, J.L. "Pharmacokinetics of Nifedipine." International J. of Clinical Pharm. Res, , 1985, 1, 63-66.

18. Robinson, D.; Mauger, J.W. "Drug Delivery Systems" American J, of Hospital Pharmacy, 1991, 48 Suppl 1, S14-S23.

19. Reichek, N. Transdermal Delivery of Nitroglycerin I. In Y.W. Chien (ed.) Transdermal Controlled Systemic Medications. Marcel Dekker, NY 1987, 227-244.

20. Polano, M. Archives Dermatology, 1976, 112, 675.

21. Hall, R.C. "Nifedipine Formulations." Medical J. of Australia 1987, 147 148150 .

22. Frishman, W.H; Sherman, D.; Feinfeld, D.A. "Innovative Drug Delivery Systems in Cardiovascular Medicine." Card. Clin. 1987, 5, 703-716.

23. Swanson, D.R.; Barclay, B.L.; Wong, P.S.; Theeuwes, F. "Nifedipine Gastrointestinal Therapeutic System." American J. of Medicine 1987, 83, 3-9.

24. Chung, M.; Reitberg, D.P.; Gaffney, M.; Singleton, W. "Clinical Pharmacokinetics of Nifedipine Gastrointestinal Therapeutic System. A Controlledrelease Formulation of Nifedipine." American J. of Medicine, 83:6 (December 1987) 10-14.

25. Siddiqui, Ovais. "Physicochemical, Physiological, and Mathematical Considerations in Optimizing Percutaneous Absorption of Drugs." Critical Reviews in Therapeutic Drug Carrier Systems, 1989, 6:1, 1-38.

26. Xu, P. and Y.W. Chien. "Enhanced Skin Permeability for Transdermal Drug Delivery: Physiological and Physicochemical Considerations." Critical Reviews in Therapeutic Drug Carrier Systems 1991, 8, 211-236.

27. Flynn, G.L.; Stewart, B. "Percutaneous Drug Penetration: Choosing Candidates for Transdermal Development." D.D.I.P 1988, 13, 169-185. 
28. Surber, C.; Wilhelm, K.P.; Hori, M.; Maibach, H.I.; Guy, R.I. "Optimization of Topical Therapy: Partitioning of Drugs into Stratum Comeum." Pharm. Res. 1990, 7, 1320-1324.

29. Rasool, A.; Hussain, A.A.; Dittert, L.W.; "Solubility Enhancement of Some Water-Insoluble Drugs in the Presence of Nicotinamide and Related Compounds." J.Pharm.Sci. 1991, 80, 387-393.

30. Janssen Pharmaceutica. Duragesic package insert. Piscataway, NJ. January 1991.

31. Calis, K.A.; Kohler, D.R.; Corso, D.M. "Transdermally Administered Fentanyl for Pain Management." Clin.Pharm 1992, 11, 22-36.

32. Roy, S.D.; Flynn, G.L. "Solubility Behavior of Narcotic Analgesics in Aqueous Media: Solubilities and Dissociation Constants of Morphine, Fentanyl and Surfentanil." Pharm.Res. 1989, 6, 147-151.

33. Hagen \& Flynn J.Pharm.Sci. 409-414.

34. Hoelgarrd, A.; Mollgaard, B. "Dermal Drug Delivery-Improvement by Choice of Vehicle or Drug Derivative." J.Cont.Rel. 1985, 2, 111-120.

35. Dean, John A., Lange's Handbook of Chemistry, Thirteenth Edition. McGrawHill Book Company NY 1985 9-90.

36. Keith, Lawrence H. and Douglas B Walters in National Toxicology Program's Chemical Solubility Compendium Lewis Publishers, Inc. Chelsea, MI 1992.

37. Rasool, A.A. Hussain, A.A.; Dittert, L.W. "Solubility Enhancement of Some Water-Insoluble Drugs in the Presence of Nicotinamide and Related Compounds. " $\mathbf{J}$ Pharm. Sci. 1991, 80, 387-393.

38. Toddywala, R.; Chien, Y.W. "Evaluation of Silicone Based Pressure-Sensitive Adhesives for Transdermal Drug Delivery II. Effect of Penetrant Lipophilicity." D.D.I.P. 1991, 17, 245-269.

39. Martin, A.; Wu, P.L.; Velasquez, T. "Extended Hildebrand Solubility Approach: Sulfonamides in Binary and Temary Solvents." J.Pharm.Sci. 1985, 74, 277-82.

40. Pieta, P.; Rava, A.; Biondi, P. "High-Performance Liquid Chromatography of Nifedipine, its Metabolites and Photochemical Degradation Products." Jour. Chrom. $1991,210,516-521$.

41. Jacobsen P.; Pederson, L.; Mickelsen, E. "Gas Chromatographic Determination of Nifedipine and One of its Metabolites Using Electron-capture Detection." Jour. Chrom. 1979, 182. 
42. Schwartz J.B.; Upton, R.A.; Lin, E.T; Williams, R.L.; Benet, L.Z. "Effect of Cimetidine or Ranitidine Administration on Nifedipine Pharmacokinetics and Pharmacodynamics." Clin.Pcl.Ther. 1988, 43, 673-680.

43. Barry B.W.; Harrison, S.M.; Dugard, P.H.. "Vapor and Liquid Diffusion of Model Penetrants Through Human Skin; Correlation with Thermodynamic Activity." Jour.Pharm. Pharmac. 1985, 37 226-236.

44. Loth, H. "Vehicular Influence on Transdermal Drug Penetration." Int.J.Pharm. $1991,68,1-10$.

45. Kondo, S.; Yamasaki-Konishi, H.; Sugimoto, I. "Enhancement of Transdermal Delivery by Superfluous Thermodynamic Potential. I. Thermodynamic Analysis of Nifedipine Across the Lipoidal Barrier" \. of Pharmacobiological Dynamics. 1987, $10,587-594$.

46. Twist, J.N.; Zatz, J.L. "A Model for Alcohol-Enhanced Permeation through Polymethylsiloxane Membranes." J.Pharm.Sci. 1986, 37 429-444.

47. Reichardt, C. Solvents and Solvent Effects in Organic Chemistry. p. 406.

48. Kier, L.B. "Quantitation of Solvent Polarity Based on Molecular Structure." J.Pharm.Sci. 1991, 70 930-933.

49. Barton, Alan F.M. in Handbook of Solubility Parameters and other Cohesion Parameters. CRC Press, Inc. Boca Raton, FL 1990.

50. Martin, Alfred, James Swarbrick and Arthur Camarata. Physical Pharmacy. Third Edition Lea \& Febiger, Philadelphia, PA. (1983).

51. Martin, Alfred, P.L. Wu and T. Velasquez, "Extended Hildebrand Solubility Approach: Sulfonamides in Binary and Ternary Solvents." J.Pharm. Sci. 1985, 74 277-282.

52. Hansen, C.; Beerblower, A. In Encyclopedia of Chemical Technology. Suppl. Vol, 2nd ed. A. Stenden, Wiley \& Sons, New York, 1972, S889-910.

53. Grulke, E.A. "Solubility Parameters and Related Phenomenon." In J. Brandrup, and E.H. Immergut, Editors. Polymer Handbook. Third Edition. John Wiley and Sons, New York. 1989 VII/519-559.

54. Hanson, C.M. and A. Beerblower, In Encyclopedia of Chemical Technology Suppl Vol, 2nd ed. Wiley, New York, N.Y., 1971, 889-910.

55. Dunn, W.J.; Block, J.H.; Pearlman, R.S. Partition Coefficient: Determination and Estimation. Pergamon Press, Elmsford, New York 1986. 
56. Vaughan, C.D. "Using Solubility Parameters in Cosmetic Formulation." Soc.Cosm. Chem. 1985, 36, 319-334.

57. Fedors, Robert. "A Method for Estimating Both the Solubility Parameters and Molar Volumes of Liquids." Polymer Engineering and Science. 1974, 14, 47-154.

58. Mullins, L.J. Chem.Rev. 1954, 54, 289-323.

59. Sloan, K.B.; Koch, S.A.M.; Siver, K.G.; Flowers, F.P. "Use of Solubility Parameters of Drug and Vehicle to Predict Flux Through Skin." J.Inv.Derm. 87 (1986): 244-252.

60. Sloan, Kenneth B, Kevin G. Siver, and Suzanne A.M. Koch, "The Effect of Vehicle on the Diffusion of Salicylic Acid Through Hairless Mouse Skin." J. Pharm Sci. 1986, 75, 744-749.

61. Pershing, L.K.; Lambert, J.D.; Knutson, K. "Mechanism of Ethanol-Enhanced Estradiol Permeation Across Human Skin in Vivo." Pharm. Res. 1990, 7, 170-175.

62. Scheuplein, Robert J. In Jarrett, A.; Editor.; Academic Press New York, 1978 The Physiology and Pathophysiology of the Skin. 1693-1730.

63. Rolfe, D. "Chemical and Physical Methods of Enhancing Transdermal Drug Delivery." Pharm. Tech. 1988, 130-140.

64. Barry B.W., "Mode of Action of Penetration Enhancers in Human Skin." J.Cont.Rel. 1983, 6, 85-97.

65. Hadgraft, J. "Penetration Enhancers in Percutaneous Absorption". Pharmacy International, 1984, 252-254.

66. Sarpot P.S.; Zatz, J.L. "Percutaneous Absorption Enhancement by Nonionic Surfactants." D.D.I.P 1987, 13 15-37.

67. Pfister, W.R.; Hsieh,D.S. "Permeation Enhancers Compatible with Transdermal Drug Delivery

Systems. II: System Design Considerations." Pharm.Tech. 1990, 14, 54-60.

68. Hori, M.; Satoh, S. "Classification of Percutaneous Penetration Enhancers: A Conceptual Diagram." In Robert L. Bronaugh and Howard I. Maibach, Editors. 1992 Percutaneous Absorption 2nd ed. Marcel Dekker, Inc. New York, N. Y. 197-214.

69. Liu, Purchum, Tamie Kurihara-Bergstrom, and William R. Good, "Cotransport of Estradiol and Ethanol Through Human Skin In Vitro: Understanding the Permeant/Enhancer Flux Relationship." Pharm.Res. 1991, 8, 938-944. 
70. Ghanem, A.H.; Mahmoud, H.; Higuchi, W.I.; Kim, Y.H. "Evaluation of the Transport Enhancing Effects of a Series of Alkanols for $\beta$-Estradiol and Other Permeants in Hairless Mouse Skin." Pharm.Res. 1988, 5, S119.

71. Knutson, K.;Krill, S.L.;Zhang, J. "Solvent-mediated Alterations of the Stratum Corneum." J.Contr.Rel 1990, 11, 93-103.

72. Friend D.R. "Cutaneous Effect of Transdermal Levonorgestrel." Food and Chemical Toxicology 1991, 29, 639-648.

73. Comfort, A.; Dinh, S.M.; Otte, J.; Shevchuk, I.; Berner, B. "Enhanced

Transport in a Therapeutic Transdermal System." Biomaterials 1990, 11, 729-733.

74. Berner, B.; Mazzenga, G.C.; Otte, J.H.; Steffens, R.J.; Juang, R.H.; Ebert, C.D. "Ethanol:water Mutally Enhanced Transdermal Therapeutic System. II. Skin Permeation of Ethanol and Nitroglycerin." J.Pharm.Sci. 1989, 78, 402-407.

75. Friend, D.R.; Catz, P.; Heller, J.; Okagaki, M. "Transdermal Delivery of Levonorgestrel. IV: Evaluation of Membranes." J.Pharm.Sci. 1989, 78, 477-80.

76. Twist, J.N.; Zatz, J.L. "A Model for Alcohol-enhanced Perneation Through Polydimethylsiloxane Membranes." J.Pharm.Sci. 1990, 79, 28-31.

77. Good, W.R.; Powers, M.S.; Campbell, P.; Schenkel, L. "A New Transdermal Delivery System for Estradiol." J.Contr.Rel. 1985, 2, 89-97.

78. Smith, RD; Robinson, D.E.; Delighieres, B.; Alberson, B.D.; Tomal, T.P.; Zinaman,M.J.; Simon, J.A. "Effects of Vehicle Supplementation on Total Estradiol Absorption from a Transdermal Estradiol Delivery System." Fert.Steri1. 1991, 56, 1029-1033.

79. Mollgaard, B.; Hoelgaard, A. "Vehicle Effect on Topical Drug Delivery II. Concurrent Skin Transport of Drugs and Vehicle Components. Acta. Pharm. Suec. $1983,20,443-450$.

80. Turi, J.S.; Danielson D.; Wotersom, J.W. J.Pharm.Sci 1979, 68, 275.

81. Polano, M.K.; Ponec, M. Arch. Dermatol. 1979, 112, 675.

82. Idson, B. "Percutaneous Absorption Enhancers." Drug and Cosmetic Industry $1985,30-33$.

83. Nomura, H.F.; Kaiho, Y.; Sugimoto, Y.; Miyashita, M.; Kato, Y. "Percutaneous Absorption of Indomethacin from Mixtures of Fatty Alcohol and Propylene Glycol (FAPG Bases) Through Rat Skin: Effect of Oleic Acid Added to FAPG Base." Chem.Pharm.Bull. 1990, 38:5, 1421-1423. 
84. Sheth, N.V.; Freeman, D.J.; , Higuchi, W.I.; Spruance, S.L. "The Influence of Azone, Propylene Glycol and Polyethylene Glycol on In Vitro Skin Penetration of Triflurothymidine." Int.Jour.Pharm. 1986, 28, 201-209.

85. Hoelgarrd, A,; Mollgaard, B. "Dermal Drug Delivery-Improvement by Choice of Vehicle or Drug Derivative." J.Contr.Rel. 1985, 2, 111-120.

86. Diem, K. and C. Letner, Documenta Geigy Scientific Tables Seventh Edition. 1970 J.R. Geigy S.A., Basel Switzerland. 604.

87. Loftsson, T.; Gildersleeve, N.; Soloman, R.; Bodor, N. "Effect of Oleic Acid on Diffusion of Drugs Through Hairless Mouse Skin." Acta Pharm. Nord. 1989, 1:1, 17-29.

88. Potts Russell O., Vivien H.W. Mak, Richard H. Guy and Michael L. Francoeur. "Strategies to Enhance Permeability via Stratum Corneum Lipid Pathways." Advanced in Lipid Research, 24 (1991): 173-210.

89. Francoeur, M.L.; Golden, G.M.; Potts, R.O. "Oleic Acid: Its Effects on Stratum Comeum in Relation to (Trans)Dermal Drug Delivery. "Pharm. Res. 1990, 7, 621627. 
TRANSDERMAL DELIVERY OF NIFEDIPINE:

FEASIBILITY STUDIES BASED

ON SOLUBILITY PARAMETERS 


\begin{abstract}
The solubilities of a hydrophobic drug, nifedipine, in a diverse panel of solvent and cosolvent systems were determined experimentally at $25^{\circ} \mathrm{C}$. The observed solubilities were examined for deviations from solubilities calculated by the Hildebrand and Scott equation. When presented graphically, the ideal solubilities showed a pattern of positive deviations from ideality. Despite its low aqueous solubility, nifedipine solubility was maximal in semipolar, aprotic cosolvents. The influence of solubility in several cosolvent systems on nifedipine permeation through hairless mouse skin was evaluated in the four cosolvent binary systems. The solubilities of nifedipine were measured in four cosolvent-dimethylisosorbide mixtures and the theoretical skin:vehicle partition coefficients were calculated. The amount of nifedipine permeated at 24 hours post application from the representative donor systems administered as suspensions were measured across nude mouse skin in vitro. For the systems studied, it was concluded that: (1) permeation is not exclusively under thermodynamic control; (2) nifedipine solubility is enhanced in moderately polar solvent systems; and (3) solubility parameter was useful in predicting the effect of donor composition on nifedipine permeation.
\end{abstract}

INTRODUCTION - The stratum corneum has been characterized as a lipoidal region that often constitutes the primary barrier to transdermal delivery. Generally, lipophilic permeants that display low polarity or poor water solubility partition more 
readily into this region of the epidermis. While most drugs are poor candidates for transdermal delivery for molecular reasons, the vehicular influence on transdermal drug penetration is well recognized ${ }^{12}$ and the choice of vehicle can materially affect permeation rates to the extent that this route becomes a viable option. ${ }^{3}$ The dihydropyridine class of calcium channel blocking agents have received attention as candidates for transdermal delivery ${ }^{4}$ with the permeation of nicardipine ${ }^{5}$ and nifedipine ${ }^{6}$ shown to be strongly influenced by the thermodynamic activity of drug in the donor phase.

A reliable means of flux prediction would be of great value in the screening of drugs and excipients. Vaughan discussed the utility of employing the solubility parameter early in the drug development process to facilitate vehicle selection in topical dosage forms. ${ }^{7}$ Several methods of predicting skin permeation have been described ${ }^{8}$ many of which require the availability of preliminary data obtained following the determination of levels of radiolabelled drug in various skin layers. Alternatively, the percutaneous absorption of some permeants have been explained in terms of molecular volume, partition coefficient, solubility, or some other related physicochemical parameter ${ }^{8}$.

Regular solution theory has been applied by several groups to describe the partitioning process in topical delivery. ${ }^{9}$ Sloan and coworkers ${ }^{10}$ used solubility parameters to estimate theoretical skin:vehicle partition coefficients of topical formulations. Their experimental design incorporated three key features. First, only saturated solutions of drug may be applied to the membrane to insure constant 
thermodynamic activity. Second, the contents of the donor and receptor compartments are rinsed and the skin is subjected to a 24 hour washout period to recover residual drug and cosolvent. Third, the effect of the initial application of donor vehicle on intrinsic membrane properties is evaluated by a second application of donor after the washout period. This study focuses on solubility theory with the ultimate goal of characterizing interactions between drug, skin and solvent that may affect the skin permeability of a drug of poor water solubility. Since nifedipine displays many of the attributes required of a drug suitable for delivery through the skin, this study employs nifedipine as a model permeant to examine the relationship between drug and vehicle on skin permeation. This is accomplished by observing the effect of donor composition upon nifedipine solubility and nifedipine permeability.

Percutaneous penetration is considered primarily a passive diffusion process that is driven by the difference between the thermodynamic activities of drug in the vehicle and the skin. "If the permeation is Fickian it may be described by equation (1) and the driving force is the concentration differential between donor and receptor.

$$
J=\frac{d M}{d t}=D S K\left(\frac{C_{d}-C_{r}}{h}\right)=P S C_{d}
$$

Equation (1) suggests that one scheme for optimizing flux is to ensure that the medicament is at its maximum thermodynamic activity within the vehicle. It has been shown that thermodynamic activity and chemical potential are simply a measure of 'escaping tendency' of a drug from a vehicle and that the higher this property, the greater the amount of drug should partition from the vehicle into the epidermis. ${ }^{12}$ 
Assuming all terms in the model remain constant, maximal flux should occur when the penetrant has achieved maximal thermodynamic activity. By definition, maximal activity occurs when solid drug is in equilibrium with drug dissolved in the vehicle. Under ideal circumstances diffusion is neither rate limited by the intrinsic dissolution rate or affected by the partitioning characteristics of the drug; and all vehicles that contain drug as a finely ground suspension also exist as a saturated solution which sustain constant escaping tendency for the duration of the experiment.

\section{METHODS}

Materials - Nifedipine, ethyl-p-amino benzoate (EPABA), propylene glycol, and fatty acids were purchased from Sigma Chemicals St Louis, MO 63178. Long chain alcohols were obtained from Janssen Chimica (Spectrum Chemical Mfg. Corp. 14422 South San Pedro St. Gardena, CA 90248). Morpholine and its analogues (Texaco Chemical Company, 4800 Fournace Place Bellaire, TX 77401); dimethylisosorbide (ICI Specialty Chemicals, Wilmington, DE 19897); ethylene and propylene oxide block copolymers (BASF Chemicals, 100 Cherry Hill Road, Parsippany, NJ 07054); Azone (Whitby Research, 2801 Reserve Street, Richmond, VA 23261); Volpols and Polychols (Croda Inc, 183 Madison Avenue, New York, NY 10016); and polyethylene glycols and silicones (Dow Coming) were donated by the respective companies.

Preparation of Solutions - In view of the high sensitivity of nifedipine to light, all experiments were carried out in a darkened room illuminated by gold fluorescent tubes (Westinghouse FC12T9 SW32W). All percentages are expressed on a 
weight:weight basis unless specified otherwise. Molecular weights obtained from average hydrophilic chain length were used in all calculations. All weight determinations were performed on a Metler A 240 balance or Perkin-Elmer Model AD-14 microbalance. Four binary cosolvent mixtures were prepared from Volpol-3 (VOL:DMI), nonoxynol-9 (NX9:DMI), polyethylene glycol 400 (PEG:DMI) and propylene glycol (PG:DMI) employing dimethylisosorbide as the secondary solvent present in the binary mixtures. The composition of these binary mixtures ranged between 0 to $100 \%$ and are expressed in terms of the mole fraction percent of the DMI component.

Differential Scanning Calorimetry - The melting point and heat of fusion for nifedipine were obtained using a Perkin Elmer Model DSC-4 calorimeter and TADS software. Determinations were in triplicate using a scan rate of 10.0 degrees per minute and a sample weight of approximately 4 milligrams in vented pans. Chromatographic conditions - All separations were performed on a Baseline 810 Chromatography Workstation (Dynamic Solutions Division of Millipore 2355 Portola Road, Ventura, CA 93003) configured as follows: a Waters Model 501 Solvent Delivery system, a Waters Intelligent Sample Processor Model 710B, and a Waters Model 484 Tunable Absorbance Detector set at 230 nanometers. Peak aquisition and integration was performed on the workstation NEC microcomputer following A/D conversion through a SIM interface. Nifedipine concentrations were determined from peak areas by the external standard method using EPABA.

A Hibar RT 250-4 LiChrosorb RP-18 (10 micron) column protected by a 
LiChrocart RP-18 (5 micron) precolumn (EM Separations, 480 Democrat Road, Gibbstown, NJ 08027) was kept at $37^{\circ} \mathrm{C}$ in a Model $\mathrm{CH}-30$ HPLC Column Heater (FIAtron Laboratory Systems, 5 I0 South Worthington, Oconomowoc, WI 53066). An isocratic mobile phase was prepared as follows: filtration of HPLC grade acetonitrile, methanol and water individually through 0.45 micron teflon filters; measurement in a ratio of $1: 1: 2$ by volume, respectively; sonication for 20 minutes in a Model T100 sonicator (National Ultrasonic Corporation, 111 Montgomery Avenue, Irvington, NJ.) and used at a flow rate of $1.3 \mathrm{ml}$ per minute.

Solubility studies - Cosolvent blends were prepared on a mole fraction basis. Donor suspensions were prepared by adding excess nifedipine to the cosolvents and agitating in a Fisher Versa-Bath S Model 224 at $37^{\circ} \mathrm{C}$ until equilibrium was obtained (generally 72 hours). The suspensions were centrifuged at 1500 rpms for 20 minutes, transferred to a 3 milliliter glass luerlock syringe fitted with a 0.2 micron Milex-FG filter (Milipore Corp. Bedford, MA 01730). The contents were expressed into amber glass tubes after having discarded the first milliter of filtrate. A tarred positive displacement syringe (Unimetrics Corporation 1853 Raymond Ave. Anaheim, CA 92801) was used to determine the density of the filtrate. Filtrates were diluted in methanol in triplicate and concentrations determined at 238 and $350 \mathrm{~nm}$ using a Hewlett-Packard 4105 Spectrophotometer.

Permeation experiments - Hairless mouse skin was chosen as a permeability batrier after initial studies showed that flux across silicone (Dow-Corning) and ethylene-vinyl acetate $(90: 10,40 \mu \mathrm{m})$ copolymer (3M Corporation) was very poor. Female hairless 
mice (26-28 grams, 8-12 weeks of age) were the progeny of four females and one male SKH-1 obtained from Charles River Laboratories. A separate fresh skin sample was used for each individual cell. Following cervical dislocation, whole thickness, intact skin from the ventral region was removed using blunt dissection and immediately placed on the diffusion cells in contact with the receptor phase.

Four vertical diffusion cells with surface area of $4.9 \mathrm{~cm}^{2}$ (Crown Glass, Somerville, NJ) were used for each experiment, keeping one as control. The receptor phases of the diffusion cells were maintained at $37 \pm 0.1^{\circ} \mathrm{C}$ with a circulating water bath. To ensure sink conditions, the receptor fluid consisted of a $6: 4(v / v)$ mixture of $0.9 \%$ sodium chloride and polyethylene glycol 400 which was replaced periodically as needed. The diffusion cell was assembled, $18 \mathrm{ml}$ of receptor solution was added, a reference (zero-hour) sample was taken, the nifedipine:cosolvent suspensions were added by weight to the donor side of the diffusion cells using a tared syringe and the donor chambers were occluded with Parafilm. All diffusion cell experiments were run using suspensions of nifedipine in the various vehicies so that the thermodynamic activity of nifedipine would remain constant throughout the study period. A control cell received the donor formulation without drug. Samples of the receptor phases (1 ml) were tranfered to $10 \mathrm{ml}$ screw top test tubes to which $40 \mu \mathrm{g}$ of EPABA had previously been added as a methanolic solution and evaporated to dryness, $50 \mu 1$ of $10 \%$ trichloroacetic acid and $4 \mathrm{ml}$ of chloroform are added, the tubes gently agitated, and centrifuged at 3000 RPM for ten minutes. The aqueous supernatent is aspirated, the organic phase evaporated to dryness and reconstituted with $1 \mathrm{ml}$ of mobile phase. 
Mass balance was performed by washing the donor compartments with $10 \mathrm{ml}$ of methanol each, taking care to remove any solid nifedipine particles and to keep the time of contact of methanol to a minimum (less than 3 minutes total). The methanol washes were combined, diluted and measured by HPLC. The receptor phase was changed and the skin kept in contact for 23 hours with fresh receptor fluid to allow any residual drug to leach out. At the end of 23 hours, the receptor phase was removed and analyzed. After another 1 hours of contact between the skin and the fresh receptor phase, the receptor phase was analyzed to assure that no more nifedipine had leached from the skin. Recoveries of approximately $90 \%$ of the original applied dose were achieved. To confirm that donor did not significantly alter barrier properties, donor was reapplied at the 48 hour mark and sampling continued until 72 hours.

We have adopted the protocol described by Sloan $^{10}$ to observe the effect of permeant solubility upon in vitro diffusion experiments in hairless mouse skin over 72 hour periods. Application of regular solution theory to predict the partitioning process requires three essential features in the experimental design: (1) saturated solutions are applied to the skin, (2) after each initial application period, a 24 hour washout period is used to determine the degree of accumulation in the skin, and (3) after the washout period, a second application of saturated drug solution is applied to determine the degree of damage to the skin.

Calculations - We assume that the properties of interest are functionally related to the the donor composition and that by changing the proportions of ingredients, $x_{i}$, the 
properties of the donor will change also. We assume the properties of the mixture to be a simple linear combination of its component cosolvents, of which the solubility parameter, $\delta$, for $n$ solvents is

$$
\delta=\sum_{i=1}^{n} \delta_{i} * x_{i}
$$

The solubility parameters were obtained from the literature ${ }^{4}$ or, if unavailable, estimated using the method proposed by Fedors ${ }^{12}$ demonstrated for nifedipine in Figure 1.

The three partial and the total solubility parameters for polyethylene glycol 400 (PEG400) are taken to be $15.8,9.1,5.2$ and $19 \mathrm{MPa}^{1 / 2}$, respectively. ${ }^{13}$ An estimate for the solubility parameter of mouse skin is $21.5 \mathrm{MPa}^{1 / 2}$, based on an estimate of 9.7-10 $\left(\mathrm{cal} / \mathrm{cm}^{3}\right)^{1 / 2}$ reported for full thickness porcine $\mathrm{skin}^{14}$ [where 1 $\left.\left.\mathrm{MPa}^{1 / 2}=2.045\left(\mathrm{cal} / \mathrm{cm}^{3}\right)^{1 / 2}\right)\right]$. The solubility parameter of nifedipine is estimated in Figure 1 to be $21.94 \mathrm{MPa}^{1 / 2}$ [i.e., $\left.10.72\left(\mathrm{cal} / \mathrm{cm}^{3}\right)^{1 / 2}\right]$. Molar volumes are equal to the molecular weights divided by the densities at $20^{\circ} \mathrm{C}$. The logarithm of the skin:vehicle partition coefficients, $\log \mathrm{PC}_{\mathrm{s}, \mathrm{v}}$, were calculated according to Sloan ${ }^{15}$ by equation (3)

$$
\log P C_{s, v_{\downarrow}}=\left[\left(\delta_{2}-\delta_{1}\right)^{2}-\left(\delta_{2}-\delta_{s}\right)^{2}\right]-\frac{V_{2}}{2.3 R T}
$$

where $\delta_{2}$ is the solubility parameter of $\mathrm{N}, \delta_{1}$ is the solubility parameter of the vehicle, $\delta_{\mathrm{s}}$ is the solubility parameter of the skin, $\mathrm{V}_{2}$ is the molar volume of $\mathrm{N}, \mathrm{R}$ is the gas constant and $\mathrm{T}$ is absolute temperature. The logarithm of the ideal mole fraction solubility of $\mathrm{N}, \log \mathrm{X}_{2}$, is calculated from the Hildebrand Equation ${ }^{16}$ where $\mathrm{X}_{2}$ is the mole fraction solubility of nifedipine at temperature $\mathrm{T}, \Delta \mathrm{H}_{\mathrm{f}}$ is the heat 


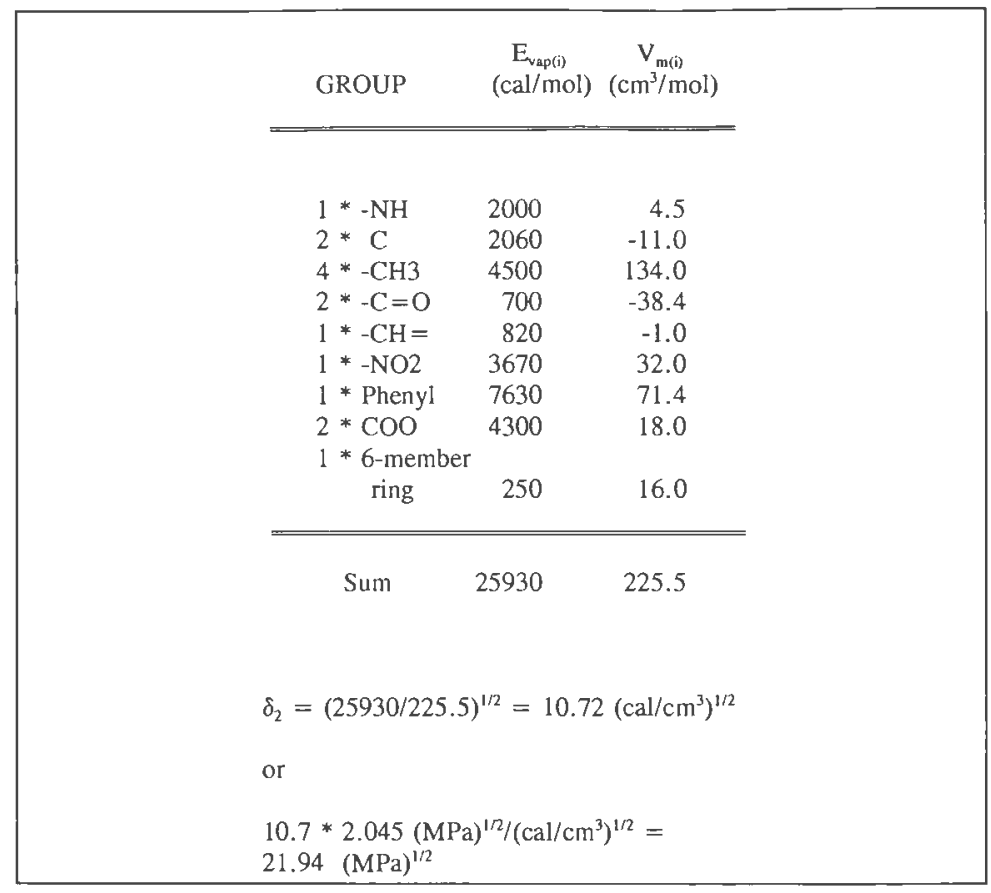

Figure 1. Estimation of nifedipine solubility parameter by Fedor's method.

$$
\log X_{2}=\frac{-\Delta H_{f}}{1364}\left(\frac{T_{m}-T}{T_{m}}\right)-\frac{V_{2} \Phi_{1}^{2}\left(\delta_{1}-\delta_{2}\right)^{2}}{1364}
$$

of fusion of the solute at its melting point $T_{n}$, and $\Phi_{1}$ is the volume fraction of the solvent.

Data analysis - The effect of mixture composition on permeation is compared by determining the average amount of nifedipine recovered in the receptor phase, $V_{r}$, 
after 24 hours. The 24 hour nifedipine receptor concentrations, $C_{r 24 h}$ were averaged and the amount permeated at 24 hours normalized for the surface area of skin in the $25 \mathrm{~mm}$ diameter cell, $\mathrm{Q}_{24 \mathrm{~h}}$, was calculated according to equation (5):

$$
Q_{24 h}=C_{r_{2 A h}} * \frac{V_{r}}{4.9 \mathrm{~cm}^{2}}
$$

and the mean $\mathrm{Q}_{24 \mathrm{~h}}$-values were used to compare penetration from different solutions.

\section{RESULTS AND DISCUSSION}

The ultraviolet (UV) spectra of nifedipine in methanolic solution obeys Beer's law between 0.22 and $80 \mu \mathrm{g} / \mathrm{ml}$ with a sharp absorption maxima at 238 and a broad band between 325 to $370 \mathrm{~nm}$. The nitrosopyridine and nitropyridine structures in Figure 2 have maxima at $280-310$ and $277 \mathrm{~nm}$, respectively, which is in agreement with literature values. ${ }^{17} 18$ The intersection of these spectra, the isosbestic point, was used as the detection wavelength for quantification by UV spectroscopy. The broad absorption band from 325 to $370 \mathrm{~nm}$ is relatively immune to solvent-induced chromic shifts and was used when other uv-absorbers interfered at the isosbestic point. While the metabolic and photodecomposition products of nifedipine are resolved under the HPLC conditions employed, they were not detected in the receptor fluid at the assay minimum level of detection $(0.04 \mu \mathrm{g} / \mathrm{ml})$. Although the metabolic fate of nifedipine in skin in vivo is unknown, we assume that metabolic breakdown products are not produced to measurable degree in the excised skin. 


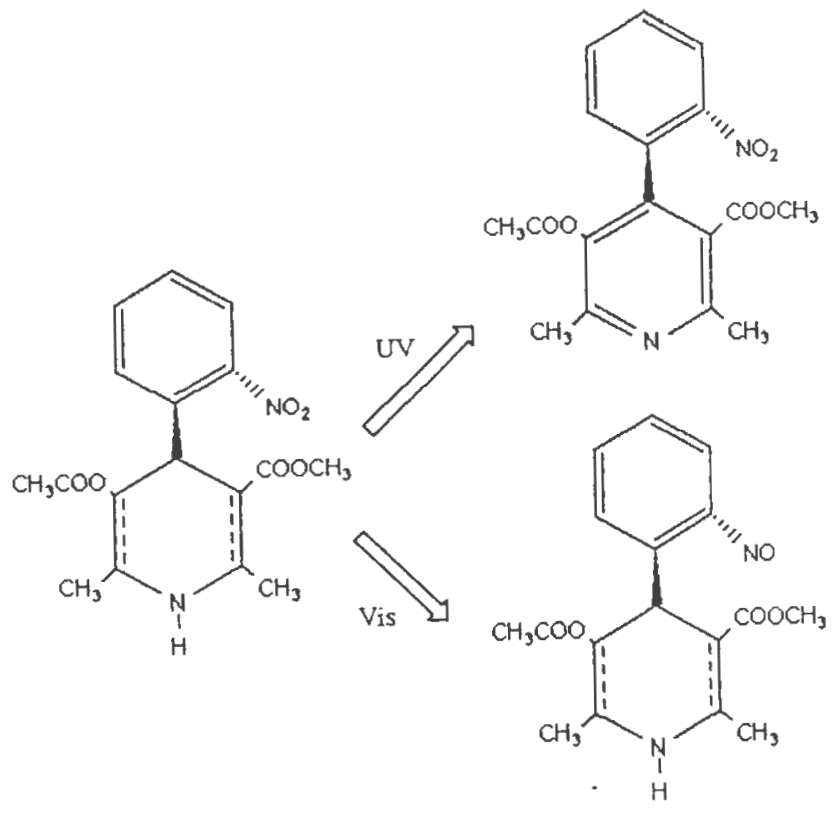

Figure 2. Structure of nifedipine and its photodecomposition products. 
The results of the solubility determinations are presented in Figure 4 . It is readily apparent that overall the solubility parameter is a poor predictor of solubility because solvents coincident with the calculated value of $21.9 \mathrm{MPa}^{1 / 2}$ for nifedipine comprise a full spectrum of low to high solubilities. The apparent shortcomings associated with the total solubility parameter are resolved when the solubility data are compared in context with either solvent $\mathrm{H}$-bonding index or hybrid solubility parameters. To account for the polar nature of solvents, the data have been plotted according to Burrell's classification of solvents ${ }^{19}$ into low, medium and high hydrogen-bonding capacity. Hanson and Beerblower ${ }^{20}$ partitioned the total solubility parameter to account for dispersion $\left(\delta_{d}\right)$, dipolar $\left(\delta_{p}\right)$ and hydrogen bonding $\left(\delta_{h t}\right)$ intermolecular forces. Partitioned in this manner the Keesom forces, described by the $\delta_{p}$ term, give rise to dipole-dipole interactions or 'orientation' forces that exert a strong influence on nifedipine solubility. When viewed in context of Burrell's $\mathrm{H}$ index, a pattern more consistent with theory emerges. Likewise, when the saturation solubility of nifedipine is plotted against the three Hanson parameters data structures analogous to those shown in Figure 4 are apparent, the most notable of which is the trend that nifedipine dissolves best in solvents with high $\delta_{p}$ values. Alternatively, no relationship between nifedipine solubility and $\delta_{d}$ was evident. Nifedipine appears to be a neutral compound with neither strong lipophilic nor hydrophilic character. Nifedipine solubility was maximal in amphiprotic (glycols, polyols, and alcohols) solvents that are somewhat polar (acetone, chloroform, dimethylisosorbide or methylene chloride) molecules having significant dipole moment. 


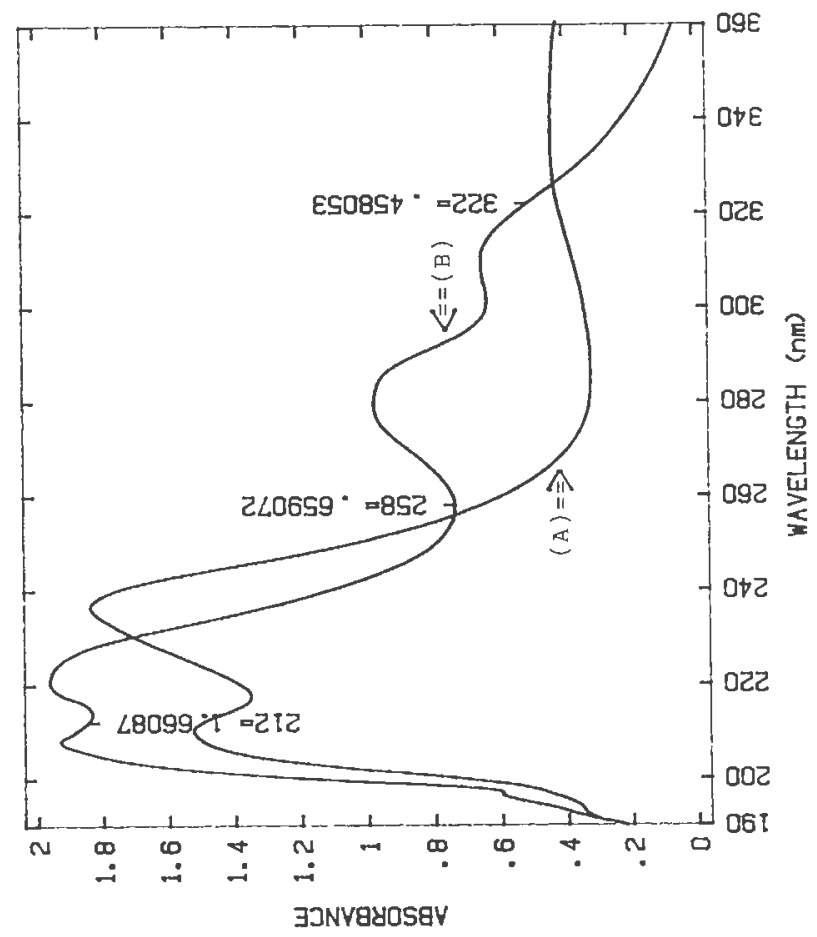

Figure 3. Ultraviolet-visible absorbance scan of nifedipine before (A) and after (B) 30 minute exposure to ambient light. 
The limited solubility of nifedipine may violate the assumption that diffusion is the rate limiting step in the permeation process. Consequently, it was important to determine the influence of equilibration time on the equilibrium saturation solubility, $\mathrm{C}_{3}$. The $\mathrm{C}_{\mathrm{s}}$ of nifedipine as a function of various equilibration times presented in Table II show no significant dependency of dissolution on equilibration period. The saturation solubility of nifedipine in water is only $0.006 \mathrm{mg} / \mathrm{ml}$ and in PEG 400 it is $99 \mathrm{mg} / \mathrm{ml}$. The saturation solubility of nifedipine in the $25 \%$ and $40 \%$ binary mixtures of PEG in normal saline are reduced to 0.240 and $0.462 \mathrm{mg} / \mathrm{ml}$, respectively. The influence of even moderate amounts of water results in a sharp decrease in nifedipine solubility in ternary mixtures of PEG:DMI:water or PG:DMI:water. As drug diffuses from the nonpolar environment of the epidermis into the hydrated layers below, the poor water solubility of nifedipine may impede its diffusion at the dermal:epidermal interface.

The ideal and experimentally observed mole fraction solubilities of nifedipine in binary cosolvents are compared in Figure 5, Figure 6 and the quaternary systems in Figure 7. Ideal mole fraction solubilities, calculated from Equation (4) consistently underestimate the solubilities determined experimentally which infer the existence of strong intermolecular attractive forces between nifedipine and cosolvent molecules. This nonideality, resulting from the second term of equation (4), violates the assumption that solute and solvent behave in a noninteracting manner and the extended Hildebrand method cannot be expected to apply where strong interactions exist. Nifedipine does not behave as an "ideal" penetrant; in neither system does the 
maximal Q-value coincide with the partition coefficient that favors optimal movement from the vehicle into the skin rendering the interpretation of $\log \mathrm{PC}_{\mathrm{s}, \mathrm{v}}$ difficult. The predictable behavior for solvents of moderate $\mathrm{H}$-bonding capacity displayed in Figure 4 suggests that the Hansen partial solubility parameter approach might offer advantages. Unfortunately the partial parameters of the stratum corneum are not known at this time. 
Figure 4. Saturation solubility $(\mathrm{mg} / \mathrm{ml})$ versus total solubility parameter (MPa) ${ }^{1 / 2}$ for the compounds studied.

KEY: acetonitrile (ACN), butanol (BOH), chloroform (CF), dichloromethane (DCM), diethylene glycol (DEG), dimethyisosorbide (DMI), dimethysulfoxide (DMSO), ethanol (ETOH), ethyl acetate (EAC), ethylene dichloride (EDC), hexane (HEX), methanol (MEOH), methylcellosolve (MC), mineral oil (MO), nonoxynol-9 (N9), PEG-5 oleyl ether (VOLPOL), polyethylene glycol (PEG400), propylene glycol (PG), piperidine (PIP), propanediol (PDIOL), tetrahydrofuran (THF). 


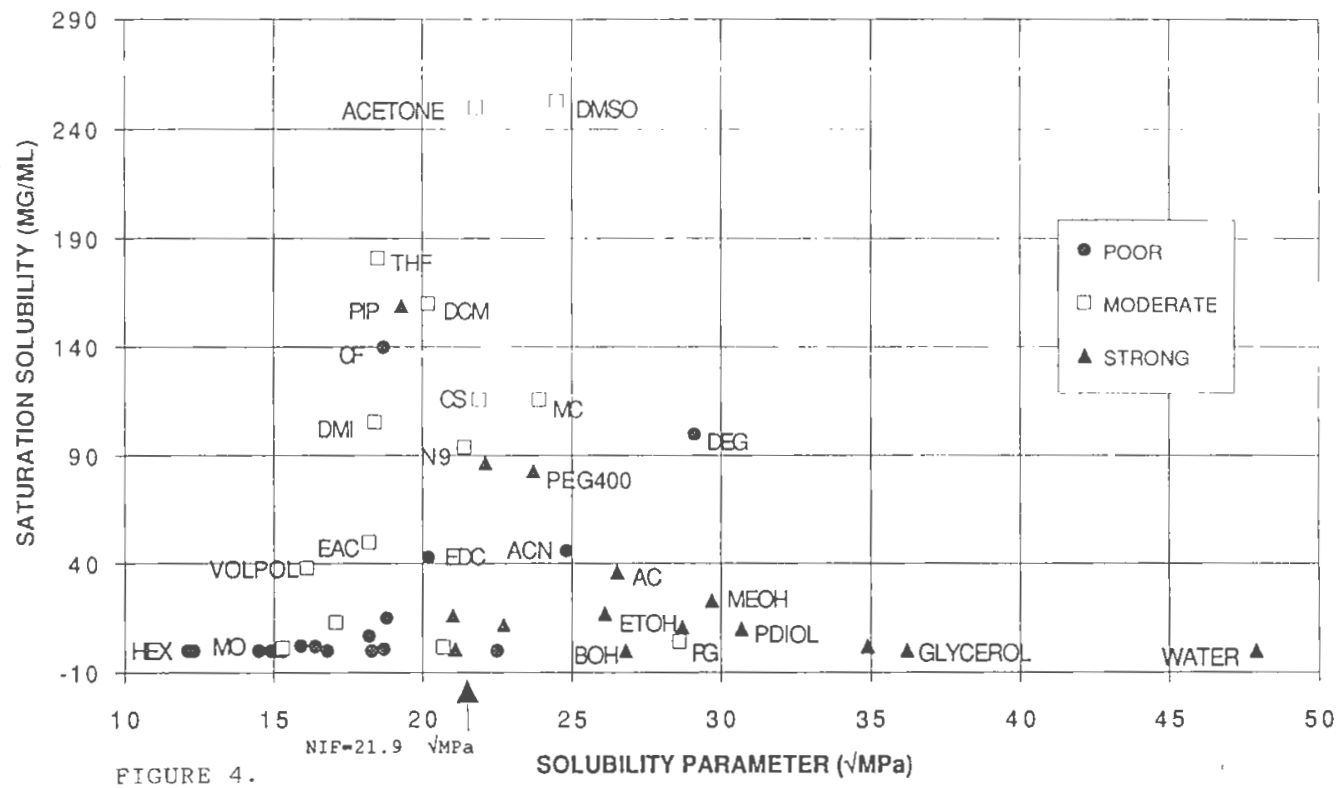


Table II. Effect of equilibration period on experimentally determined saturation solubilities of nifedipine in several solvents.

\begin{tabular}{|c|c|c|c|c|c|}
\hline \multirow{3}{*}{ Solvent ${ }^{n}$} & \multicolumn{5}{|c|}{ Equilibration Period (days) } \\
\hline & 0.5 & 1 & 3 & 7 & 30 \\
\hline & \multicolumn{5}{|c|}{ Equilibrium Saturation Solubility ( $\mathrm{mg} / \mathrm{ml})^{\mathbf{b}}$} \\
\hline DMI & $\begin{array}{c}88.44 \\
(1.4)\end{array}$ & $\begin{array}{l}89.75 \\
(2.3)\end{array}$ & $\begin{array}{l}88.15 \\
(2.0)\end{array}$ & $\begin{array}{c}94.33 \\
(5.3)\end{array}$ & $\begin{array}{c}90.18 \\
(2.8)\end{array}$ \\
\hline PEG & $\begin{array}{l}82.57 \\
(3.6)\end{array}$ & $\begin{array}{l}83.20 \\
(2.3)\end{array}$ & $\begin{array}{c}82.97 \\
(2.9)\end{array}$ & $\begin{array}{c}83.75 \\
(1.2)\end{array}$ & $\begin{array}{c}84.14 \\
(2.0)\end{array}$ \\
\hline PG & $\begin{array}{c}9.2 \\
(2.4)\end{array}$ & $\begin{array}{l}10.1 \\
(3.5)\end{array}$ & $\begin{array}{l}11.8 \\
(2.2)\end{array}$ & $\begin{array}{c}9.7 \\
(1.7)\end{array}$ & $\begin{array}{l}10.7 \\
(3.6)\end{array}$ \\
\hline Water & 0.014 & 0.013 & 0.015 & 0.017 & 0.014 \\
\hline
\end{tabular}

${ }^{2}$ Key: dimethylisosorbide (DMI), polyethylene glycol (PEG), propylene glycol (PG); baverage of three determinations ( \pm standard deviation); ${ }^{c}$ single determination;

Further evidence of significant Keesom interaction is found in the disparity of partition coefficients obtained from various lipophilic phases. The partition ratio varies greatly depending on the material chosen to represent the lipophillic phase (approximately 10:1 and 10000:1, when measured in cyclohexane-aqueous buffer and octanol:aqueous buffer ( $\mathrm{pH} 7$ ) systems, respectively). The disparity between the partition coefficient measured in octanol versus hexane may be attributed to the 


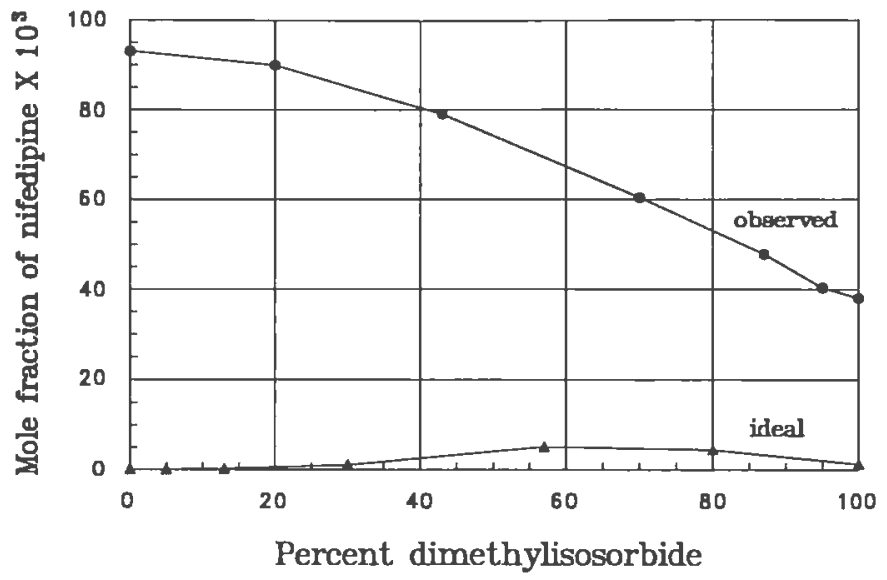

Figure 5. Nifedipine solubility in polyethylene glycol 400:dimethylisosorbide binary cosolvent mixtures.

Keesom interactions deemed present based on the pattern in the solubility results presented in Figure 4. Although nifedipine does not readily infiltrate environments of strong hydrogen bonds, there is a distinct affinity for solvents with dipole-dipole intermolecular attractions. For example, in Table III, nifedipine has a high solubility in the morpholine cogenors tested. These molecules have significant dipole nature and suspensions of nifedipine in the morpholine derivatives all demonstrated high fluxes.

The environment of the stratum corneum is primarily lipophilic in nature and it has been suggested that 'best' candidates for transdermal delivery are lipophilic molecules. Nifedipine, with neglible water solubility, a large octanol:buffer partition 


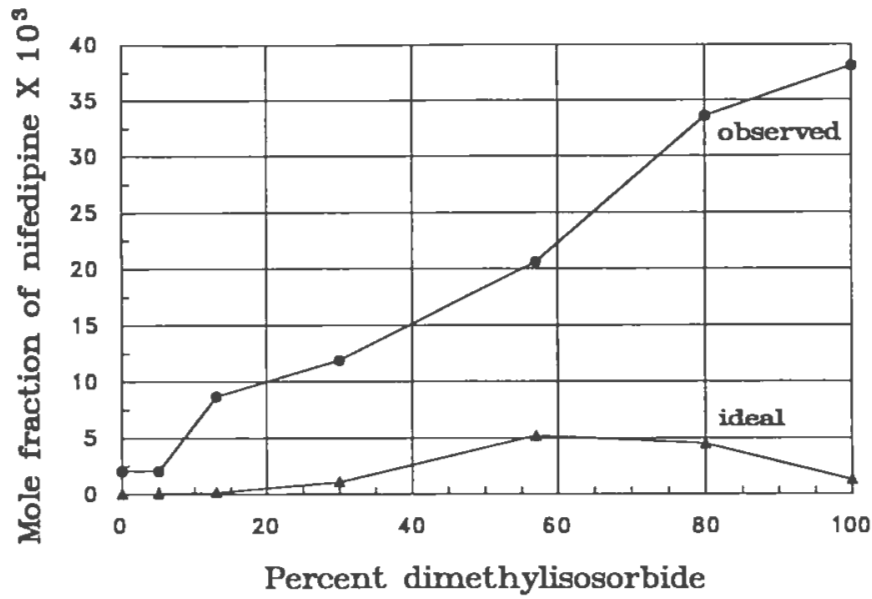

Figure 6. Nifedipine solubility in propylene glycol:dimethylisosorbide binary cosolvent mixtures.

coefficient, and a total solubility parameter matching that of the skin, appears at first glance to posses the prerequisite qualities of a 'good' candidate for transdermal therapy because of its 'lipophilic' nature. However, our solubility results clearly show that nifedipine appears to interact strongly with solvents primarily via Keesom forces which accounts for a poor solubility in lipids (hydrocarbons, fixed oils, etc.) and a low hexane:buffer partition coefficient. In addition, recovery studies show that only small amounts $(<10 \%)$ of the total dose applied partition into and are retained by the excised skin. Furthermore, our results of differential scanning calorimetry shown in Figure 8 reveals a melting point and enthalpy of fusion of nifedipine that is uncharacteristically high for those compounds in Table I on page 7 generally 
classified as lipophilic. In view of the many contradictions that can be ascribed to attempting a lipophilic-hydrophilic designation for nifedipine, a relationship between

Table III. Solubility and diffusion from nifedipine suspended in morpholine derivatives.

\begin{tabular}{||l|c|c|c||}
\hline \multicolumn{1}{|c|}{ Solvent } & $\delta_{\mathrm{T}}$ & $\mathrm{C}_{\mathrm{s}}$ & $\mathrm{J}$ \\
\hline Morpholine & 9.36 & 266 & $12.2 \pm 2.9$ \\
\hline Methyl morpholine & 9.49 & 228 & $14.9 \pm 2.0$ \\
\hline Ethyl morpholine & 9.33 & 223 & $9.9 \pm 0.7$ \\
\hline $\begin{array}{l}\text { Aminopropyl } \\
\text { morpholine }\end{array}$ & 9.22 & 360 & $5.3 \pm 0.9$ \\
\hline
\end{tabular}

KEY: $\delta_{\mathrm{T}}=$ total solubility parameter expressed in units of $(\mathrm{MPa})^{1 / 2} ; \mathrm{C}_{\mathrm{s}}=$ saturation solubility of nifedipine as $\mathrm{mg} / \mathrm{ml} ; J=f l u x$ as $\mu \mathrm{g} / \mathrm{h} \bullet \mathrm{cm}^{2}$; (permeation results obtained over the interval $=0$ to 24 hours). Results represent the mean of 3 determinations \pm standard deviation.

$\log \mathrm{PC}_{\mathrm{s}, v}$ and nifedipine skin permeability is unlikely. In the systems studied, prediction of drug permeability based on generalizations regarding lipophilicity of the donor phase relative to that of skin are inappropriate for in this case 'lipophilicity' is a label that fails to classify. The predominate intermolecular attractive forces in lipidlike molecules originate from Van der Walls forces which are described by the dispersion hybrid parameter. The primary pathway through the stratum corneum is generally attributed to passive diffusion through intercellular lipids. The lack of a 


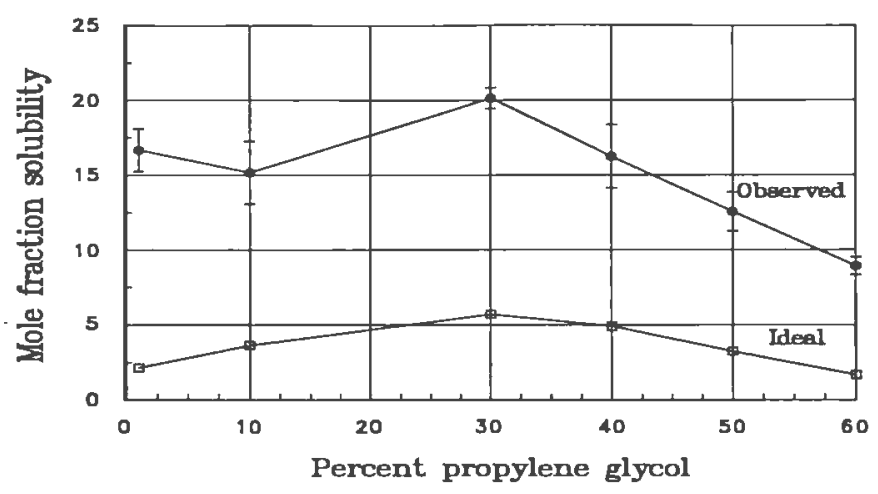

Figure 7. Nifedipine solubility in propylene glycol, oleic acid, ethanol and dimethylisosorbide quaternary cosolvent mixtures.

relationship between nifedipine solubility and solvent $\delta_{\mathrm{d}}$ values imply minimal interaction between nifedipine and intercellular lipid. Likewise, partitioning of nifedipine into the well-hydrated dermis is predicted to be low due to the presence of extensive strong intermolecular hydrogen bonding.

Table IV contains the composition and permeation results from binary systems constituting a broad range of solubility parameters that bracketed the point of maximal nifedipine solubility. When these systems were reapplied to the cells following the washout period, no difference was observed in the pre and post treatment $Q$ values and it is assumed that their effect on the intrinsic barrier properties of the skin are insignificant. Highest permeability is expected at the point of maximum thermodynamic activity and if solubility is a reliable indicator of activity, then theory 


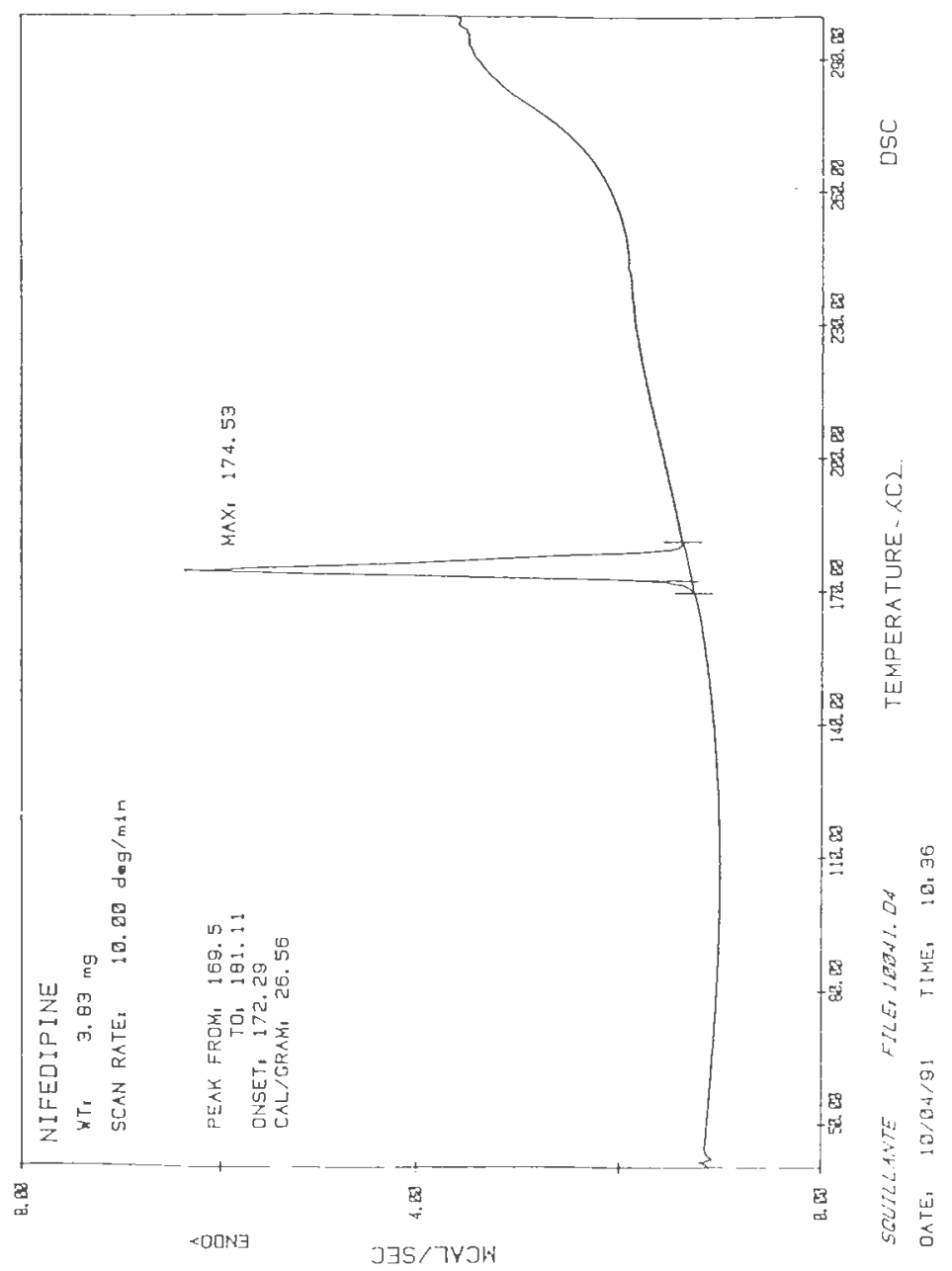

Figure 8. Differential scanning calorimeter thermogram for nifedipine. 
Table IV. Saturation solubility in and permeability of nifedipine from binary mixtures prepared by the addition of propylene glycol or polyethylene glycol in dimethylisosorbide.

\begin{tabular}{||c|c|c|c|c||}
\hline \hline $\mathrm{X}_{\mathrm{i}}$ & $\delta_{\mathrm{T}}$ & $\mathrm{C}_{\mathrm{s}}$ & $\mathrm{J}$ & $\begin{array}{c}\text { Nifedipine } \\
\text { permeated }\end{array}$ \\
\hline Fraction of PG in DMI & \\
\hline 0.00 & 18.5 & $85.0 \pm 2.5$ & $0.25 \pm 0.08$ & $29 \pm 9$ \\
\hline 0.20 & 20.5 & $81.5 \pm 2.7$ & $0.17 \pm 0.71$ & $20 \pm 84$ \\
\hline 0.43 & 22.8 & $57.5 \pm 0.2$ & $1.46 \pm 0.60$ & $50 \pm 71$ \\
\hline 0.70 & 25.6 & $40.5 \pm 5.0$ & $0.61 \pm 0.18$ & $172 \pm 21$ \\
\hline 0.87 & 26.0 & $19.1 \pm 0.4$ & $4.45 \pm 1.02$ & $523 \pm 120$ \\
\hline 0.95 & 27.3 & $9.2 \pm 0.01$ & $3.84 \pm 1.46$ & $455 \pm 172$ \\
\hline 1.00 & 28.1 & $10.0 \pm 0.6$ & $1.86 \pm 0.76$ & $219 \pm 89$ \\
\hline Fraction 0 of PEG in DMI & & $165 \pm 27$ \\
\hline 0.00 & 18.5 & $85 \pm 2.5$ & $0.25 \pm 0.08$ & $29 \pm 9.4$ \\
\hline 0.05 & 18.7 & $98 \pm 1.0$ & $1.42 \pm 0.23$ & $165 \pm 18$ \\
\hline 0.13 & 19.1 & $105 \pm 2.8$ & $1.51 \pm 0.15$ & $177 \pm 13 \pm 13$ \\
\hline 0.30 & 19.8 & $106 \pm 3.1$ & $2.33 \pm 0.11$ & $275 \pm 13$ \\
\hline 0.57 & 21.5 & $99 \pm 3.6$ & $5.78 \pm 3.47$ & $680 \pm 408$ \\
\hline 0.80 & 22.7 & $92 \pm 2.8$ & $2.55 \pm 0.87$ & $300 \pm 102$ \\
\hline 1.00 & 23.8 & $85 \pm 1.5$ & $0.09 \pm 0.02$ & $10 \pm 2$ \\
\hline \hline
\end{tabular}

KEY : $\mathrm{X}_{\mathrm{j}}=$ mole fraction of PG or PEG in DMI; $\delta=$ solubility parameter expressed in units of $(\mathrm{MPa})^{1 / 2} ; \mathrm{C}_{\mathrm{s}}=$ saturation solubility of nifedipine in $\mathrm{mg} / \mathrm{ml} ; \mathrm{J}=\mathrm{flux}$, in units of $\mu \mathrm{g} / \mathrm{h} \cdot \mathrm{cm}^{2}$ "Cumulative amount of nifedipine in $\mu \mathrm{g}$ permeated over a 24 hour period (permeation results obtained over the interval $=0$ to 24 hours). Results represent the mean of 3 determinations \pm one standard deviation.

suggests that maximum solubility should occur when the solubility parameter of solute 
and solvent are equal. If permeation is mostly controlled by thermodynamic activity and if solubility measurements are a reliable indicator of activity, we would expect all PEG:DMI binary mixtures to yield equal permeation rates, which was clearly not the case. These results illustrate a general lack of usefulness of $\mathrm{C}_{\mathrm{s}}$ values in predicting skin permeation. The premise that flux is directly proportional to activity is an oversimplification that ignores the partitioning behavior of the permeant. While high concentrations of permeant in the donor phase relative to skin should promote high diffusion rates, equation (1) predicts that maximal flux should be achieved when the product of drug concentration in the vehicle, $\mathrm{C}_{\mathrm{v}}$, and $\mathrm{PC}_{\mathrm{s}, \mathrm{v}}$ is optimal so that permeation is as much a function of the skin:vehicle partition coefficient.

Equation (2) and its extensions have been successfully applied to predict the effect of vehicle on salicylic acid ${ }^{15}$ and 5-fluorouracil ${ }^{21}$ delivery through mouse skin. Figure 9 summarizes the relationship between the theoretical skin vehicle partition coefficient, $\log \mathrm{PC}_{\mathrm{s}, v_{v}}$, and the total amount of nifedipine permeated in the 24 hour study period, $Q_{24 h}$, for the PEG:DMI binary systems. The data for the PG:DMI binary systems are treated in a similar manner in Figure 10. Fluxes were inversely dependent on drug solubility in the vehicles with minimum fluxes corresponding to the point where $\delta_{2}=\delta_{1}$ as predicted by equation (3). Likewise, in a study of the permeation of estradiol from varied concentrations of PEG 400, Valia and $\mathrm{Chien}^{22}$ found that drug permeation rates decreased as the PEG level increased (i.e., estradiol solubility in donor increases). The solubility of nifedipine is approximately constant in the PEG:DMI binary systems. The modest permeation rates from PEG:DMI 
systems did not differ significantly $(p=0.05)$ due to the high variability in the data. The trend for PG:DMI binary cosolvent mixtures of low solubility (high \% PG) to display higher fluxes is also consistent with equation (3). The corollary between the works cited and our results suggests that drug solubility in the donor is itself a poor indicator of permeation. Apparently, an optimal point for penetration, corresponding to the product of $\mathrm{C}_{\mathrm{v}}$ and $\mathrm{PC}_{\mathrm{s}, \mathrm{v}}$, exists after which drug is less easily released from donor vehicles displaying high nifedipine solubilities.

Khalil and Martin ${ }^{23}$ showed that the rate of transfer of salicylic acid from one phase to a second phase was directly dependent on the differences between the solubility parameter of the solute and the two phases. Furthermore, the closer the solubility parameter of salicylic acid was to that of the membrane, the faster was the rate of transfer of acid. The solubility parameters of DMI, nifedipine, porcine stratum corneum, and propylene glycol are $18.4,20.5,20.5$, and $28.6 \mathrm{MPa}^{1 / 2}$, respectively. If large differences in the solubility parameter between permeant and donor accelerate diffusion, better fluxes are predicted from binary mixtures high in PG and low DMI. The results presented in Figure 10 are consistent with these predictions. Permeation was fourfold greater from all PG:DMI formulations $(\delta=18$ 29) compared to PEG:DMI formulations $(\delta=18-25)$.

This discussion has considered the diffusion exclusively from a static perspective that gives rise to experimental observation not compatible with that anticipated from theory. However, when considered dynamically, predicted shifts in $\delta_{s}, \mathrm{X}_{2}$, and $\mathrm{PC}_{\mathrm{s}, \mathrm{v}}$ caused by codiffusion of the DMI and PG components explain these 
inconsistencies rather well. If nifedipine flux is dependent on the establishment of a hypothetical cosolvent gradient within the dermis, it would be expected to be transient and reversible in nature. Small amounts of DMI could transiently alter the hydrophilic layers, functioning as a bolus to introduce nifedipine into the skin. A rapid depletion of the DM component would explain why amount of drug permeated versus time profiles for systems in Figure 9 high in PEG (which does not traverse the skin yet has comparable solvent strength for $\mathrm{N}$ ) have low $\mathrm{Q}_{24 \mathrm{~h}}$ values.

A further assumption in this scheme is that the vehicle should in no way alter the intrinsic properties of the skin. This discussion, based entirely on the presumption of static conditions, assumes that $\delta_{1}$ remains constant and disregards the codiffusion of vehicle into and moisture out of the skin. Other studies with hydrophobic drugs reveal that a complex interplay among drug, solvent, and skin exists in which linkage between drug and solvent permeation has unpredictable net results on permeation, illustrating the necessity for the measurement of the concurrent transport solvent levels. ${ }^{24}$ DMI and PG are well absorbed through the skin and it is quite possible that at steady state, a gradient of cosolvent exists down through the various skin regions. Good et al. ${ }^{25}$ observed a correlation between the flux of ethanol and estradiol. While there was no correlation between skin permeability and solubility in the vehicle, estradiol flux was linked to ethanol flux. Mollgaard and Hoelgaard ${ }^{26}$ measured the simultaneous skin permeation of estradiol, metronidazole, and propylene glycol and found that the accelerant properties were linked to ease of solvent penetration. 


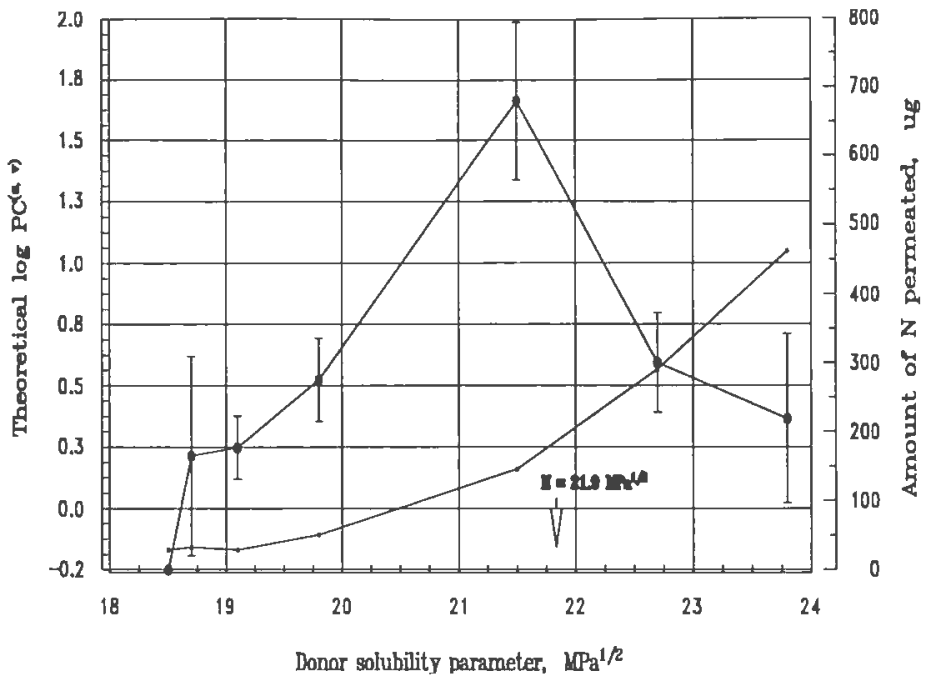

Figure 9. Relationship between theoretical log skin-vehicle partition coefficient, amount permeated over 24 hours and solubility parameter for PEG:DMI binary mixtures.

Gross observations, using capillary tube measurements of the height of PG and DMI levels in the donor compartment, reveal that both solvents readily permeate the skin. If donor composition changes over the study period, so must donor solubility parameter and the calculated partition coefficients. For the PG:DMI systems (and, to some degree, the PEG:DMI systems) following application of the donor suspension, formulations with higher percentages of DMI initially have relatively large differences between the amount of drug in the skin and vehicle (i.e., high $\mathrm{C}_{\mathrm{v}}-\mathrm{C}_{\mathrm{s}}$ values), creating a concentration gradient for movement from vehicle into skin. With time, DMI and, 


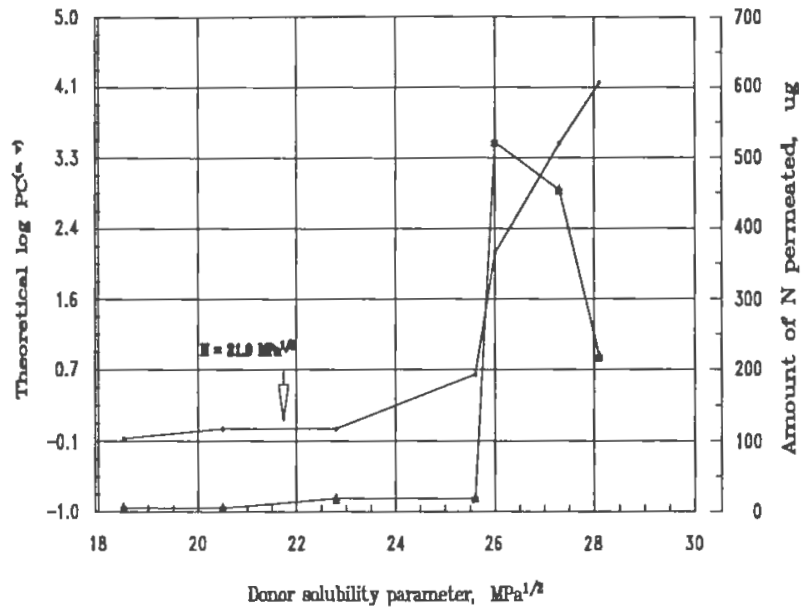

Figure 10. Relationship between theoretical log skin-vehicle partition coefficient, amount permeated over 24 hours and solubility parameter from PG:DMI binary mixtures.

to a lesser extent, PG codiffuse through the skin which decreases the ratio of DMI relative to $\mathrm{PG}$ in the vehicle, lowering drug saturation solubility in the donor.

Codiffusion of solvent with drug would alter the $\mathrm{PC}_{s, v}$ in a way that favors higher permeation rates for formulations from which all DMI is penetrated in the study period. Better flux values were in fact observed in the DM-poor PG:DM formulations. Based on what is known about the epidermal and dermal layers and the physicochemical properties of the drug and cosolvent, it is tempting to assign a linkage between drug and solvent movement. The precise information regarding the rate and extent of DMI movement through the skin necessary for this type of analysis is not available at this time. 
In summary, the transdermal permeation of nifedipine was examined in terms of its solubility profile. Although thermodynamic diffusant activity was a poor predictor of nifedipine permeability, it was possible to predict in a relative manner permeation response based on the initial donor solubility parameter. These results support the hypothesis that a linkage between nifedipine and vehicle codiffusion may exist and further studies to establish the nature of this dependency are underway. 


\section{REFERENCES}

1. Loth, H. "Vehicular Influence on Transdermal Drug Penetration". Int.J.Pharm 1991, 68(1), 1-10.

2. Bennett, S.L.; Barry, B.W.; Woodford, B. "Optimization of Bioavailability of Topical Steroids: Nonnucleated Penetration Enhancers Under Thermodynamic Control." J.Pharm.Pharmacol. 1985, 37, 298-304.

3. Guy, Richard H. and Jonathan Hadgraft. "Selection of Drug Candidates for Transdermal Drug Delivery: Developmental Issues and Research Initiatives." In Transdermal Delivery Systems New York, N.Y.: Marcel Dekker, Inc., 1989. 59-82.

4. Diez, I.; Colom, H.; Moreno, J.; Obach, R.; Peraire, C.; Domenech, J. "A Comparative In Vitro Study of Transdermal Absorption of a Series of Calcium Channel Antagonists." J.Pharm.Sci. 1991, 80, 931-934.

5. Seki, T.; Sugibayashi, K.; Morimoto, Y. "Effect of Solvents on the Permeation of Nicardipine Hydrochloride though Hairless Rat Skin." Chemical Pharmaceutical Bulletin. 1987, 35, 3054-3057.

6. Kondo, S.; Yamasaki-Konishi, H.; Sugimoto, I. "Enhancement of Transdermal Delivery by Superfluous Thermodynamic Potential. II. In Vitro-In Vivo Correlation of Percutaneous Nifedipine Transport." J.Pharmacobio.Dyn. 1987, 10, 662-668.

7. Vaughan, C.D. "Using Solubility Parameters in Cosmetic Formulation." I. Soc. Cosm. Chem. 1985, 36, 319-334.

8. Tojo, K.; Lee, A.C. "A Method for Predicting Steady-state Rate of Skin Penetration In Vivo." J.Inv.Derm. 1989, 92, 105-108.

8. Potts, R.O.; Guy, R.H. "Predicting Skin Permeability." Pharm. Res.. 1992, 9, 663-669.

9. Surber, C.; Wilhelm, K.P.; Hori, M.; Maibach, H.I.; Guy, R. "Optimization of Topical Therapy: Partitioning of Drugs into Stratum Corneum." Pharm. Res. 1990, $7: 12,1320-1324$.

10. Sloan, K.B., ed. "Use of Solubility Parameters from Regular Solution Theory to Describe Partitioning-Driven Processes." in Prodrugs. 179-204 Marcel Dekker, Inc. New York, NY. 1992.

11. Woodford R.; Barry, B.W. "Optimization of Bioavailability of Topical Steroids: Thermodynamic Control." J.Inv.Derm. 1982, 79, 388-391. 
12. Kondo, S.; Sugimoto, I. "Enhancement of Transdermal Delivery by Superfluous Thermodynamic Potential. I. Thermodynamic Analysis of Nifedipine Transport Across the Lipoidal Barrier." J.Pharmacobio.Dyn. 1987, 10, 587-594.

11. Barton, A.F.M. Chem. Rev. 1975, 75, 731.

12. Fedors, Robert. "A Method for Estimating Both the Solubility Parameters and Molar Volumes of Liquids." Polymer Engineering and Science. 1974, 14, 147-154.

13. Dow Chemical Company, Performance Products Department, Polyglycols Technical Sevices and Development B1605, 2301 N. Brazosport Blvd, Freeport, TX 77541-3257. (Trudy Wells (409) 238-9284, personal communication).

14. Liron, Z.; Cohen, S. "Percutaneous Absorption of Alkanoic Acids. II: Application of Regular Solution Theory." J.Pharm.Sci 1984, 73, 538-542.

15. Sloan, K.B.; Siver, K.G.; Kochand, A.M. "The Effect of Vehicle on the Diffusion of Salicylic Acid Through Hairless Mouse Skin." J.Pharm.Sci 1986, 75:8, 744.

16. Alfred Martin, James Swarbrick and Arthur Camarata. Physical Pharmacy, 3rd Ed. Lea \& Febiger, Philadelphia, PA. (1983).

17. Ali, Syed Laik. Nifedipine in Analytical Profiles of Drug Substances Volume 18. Klaus Florey, Ed. Academic Press, Inc. San Diego, CA (1983).

18. Majeed I.A.; Murray, W.J.; Newton, D.W.; Othman, S.; Al-Turk, W.A. "Spectrophotometric Study of the Photodecomposition Kinetics of Nifedipine." J.Pharm.Pharmac. 1987, 39:12, 1044-1046.

19. Brandup, J. and E.H. Immergut, Polymer Handbook Wiley, New York 1975 IV 337.

20. Hansen, C.M. J. Paint Technol. 1967, 39, 505.

21. Sheretz, E.F.; Sloan K.B.; McTiernan, R.G. "Transdermal Delivery of 5Fluorouracil Through Skin of Hairless Mice and Humans In Vitro: a Comparison of the Effect of Formulations and Prodrug." Arch. Dermatol. Res. 1990, 282(7), 463-8.

22. Valia, K.H.; Chien, Y.W. "Long Term Skin Permeation Kinetics of Estradiol I. Effect of Drug Solubilizer-Polyethylene Glycol 400." D.D.I.P. 1984, 10, 951.

23. Khalil, S.A.; Martin, A. "Drug Transport through Model Membranes and its Correlation with Solubility Parameters." J.Pharm.Sci. 1967, 56, 1225-1233. 
24. Hori, M.; Maibach, H.; Guy, R. "Enhancement of Propranolol and Diazepam Skin Absorption In Vitro. II: Drug, Vehicle, and Enhancer Penetration Kinetics." J.Pharm.Sci. 1992, 81(4), 330-333.

25. Good W.; Powers M.; Campbell P.; Schenkel L. "A New Transdermal Delivery System for Estradiol." J.Cont.Rel. 1985, 2, 89-97.

26. Moligaard, B.; Hoelgaard, A. "Vehicle Effect on Topical Drug Delivery: II. Concurrent Skin Transport of Drugs and Vehicle Components." Acta. Pharm. Suec. $1983,20,443-450$. 
APPLICATION OF SOLUBILITY THEORY

TO COMPONENT SELECTION

OF A TRANSDERMAL DELIVERY SYSTEM

FOR NIFEDIPINE 


\begin{abstract}
In vitro experiments using suspensions of nifedipine $(\mathrm{N})$ in mixtures of solvents were applied to fresh excised hairless mouse skin. The ability of penetration enhancer:solvent combinations of homologous members of alcohols and alkanoic acids in combination with Azone and isopropyl myristate to accelerate delivery rates of nifedipine was evaluated. Many of the alkanoic acids had a beneficial effect on permeation that was intensified in the presence of vehicles which readily pass the epidermis. Four factors - propylene glycol (PG), oleic acid (OA), ethanol (ET) and dimethylisosorbide (DM) - strongly affected nifedipine permeation. The positive synergistic effects obtained by combining one or more enhancers which act directly on the epidermis with solvents that improve drug partitioning in the skin resulted in flux values fourfold higher than that required to deliver the equivalent oral daily dose. Mechanical stripping of the skin showed that the stratum corneum was not the sole source of diffusional resistance. These results suggest that it may be feasible to deliver clinically useful amounts of nifedipine percutaneously by the judicious choice of vehicle composition.
\end{abstract}


Introduction - Successful systems must overcome the skin's natural ability to provide an efficient barrier to the ingress of foreign materials. Consequently, transdermal delivery has been considered feasible only for drugs for which the daily dose is of the order of a few milligrams. ${ }^{1}$ In the case of hydrophobic drugs it remains unclear how to determine a priori the optinal features required in a transdermal delivery system to best overcome this barrier. Since selection of components is the single most important decision in the design of topical drug delivery systems, information of this nature is crucial in the early phases of dosage form design. The pharmacokinetic (short elimination half-life, moderately low daily dose and chronic use) and pharmacological properties of nifedipine (erratic and unreliable absorption following oral or sublingual delivery) are well suited to transdermal delivery systems (TDS). The present gap in knowledge dictates a systematic inquiry of those physicochemical properties of both drug and vehicle that limit drug suitability so that the rational formulation of new generations of transdermal delivery systems can occur.

Of the several layers of epidermis, the stratum corneum (SC) is recognized to be the primary barrier to transdermal diffusion. Although only 10 to $15 \%$ of the total SC mass is composed of lipids, these lipids largely dictate the overall skin permeability properties. However, the skin is a heterogenous laminate which, due to its composition, may offer differential influence on overall skin permeability. Diffusion through this heterogenous milieu can be envisioned as a series of partitions starting in the lipophilic environment of the stratum comeum to a hydrophilic one in the dermal region and the adjacent systemic circulation. The solubility parameter of 
nifedipine and porcine stratum comeum are similar $(\approx 10 \mathrm{Hil})$, consequently, nifedipine should readily penetrate stratum corneum intercellular lipids. It had been previously determined that nifedipine $(\mathrm{N})$, despite optimal thermodynamic conditions favoring its diffusion from donor to receptor in vitro, does not penetrate the skin at rates sufficient to permit clinically useful transdermal application, presumably due to its poor solubility in both lipophilic and hydrophilic media. ${ }^{2}$ Solubility theory offers insight as to what attributes are essential in vehicle components for nifedipine. The solubility profile is such that further penetration into the deeper layers is not favorable due to the markedly poor affinity for systems structured by extensive hydrogen bonding. When viewed in this simplistic manner, one would expect the diffusion of a hydrophobic molecule to precede readily through the epidermis and diffuse poorly through the dermal layers. It is likely that for drugs with poor aqueous solubility diffusional resistance may reside in the well hydrated dermal layers so that the choice of enhancer would likely be much different than more soluble permeants. This study seeks to characterize this partitioning process in order to gain a better understanding of the molecular basis of permeability enhancement in percutaneous absorption.

Methods - A series of experiments was set up using vehicles consisting of dimethylisosorbide $(\mathrm{DM})$ mixed with equimolar amounts of even-rlumbered alkanols ${ }^{\natural}$ and alkanoic acids ${ }^{c}(8-18 \mathrm{C})$. In-vitro screening was performed using the following

\footnotetext{
"ICI Specialty Chemicals, Wilmington, DE 19897.

bJanssen Chimica 14422 South San Pedro St Gardena, CA 90248.

'Sigma Chemicals St Louis, MO 63178.
} 


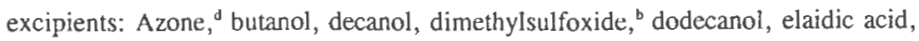
ethanol, isopropyl myristate, ${ }^{2}$ isopropyl palmitate, ${ }^{2}$ linoleic acid, linolenic acid, methanol, myristic acid, nonanol, oleic acid, oleyl alcohol, palmitic acid, palmitoleic acid, polyethylene glycols ${ }^{c}$, propylene glycol, ${ }^{\circ}$ stearic acid, and undecylenic acid. All cosolvent systems were prepared and measured on a molar basis.

The saturation solubility of nifedipine in each system was determined by standard procedures. Unless stated otherwise, mixtures were prepared by combining the long chain acids and alcohols with either dimethylisosorbide or PEG 400 such that each contained $10.6 \mu \mathrm{mol}$ of enhancer. Donor formulations were prepared by adding a moderate (10\%) excess of nifedipine to the cosolvent mixtures and agitating for 72 hours at $37^{\circ} \mathrm{C}$. The relative potencies of the various components were compared by a strategy discussed by Snee and Marquard ${ }^{3}$ which determines the most important components in a multi-component blended system. The 15 possible quaternary mixtures of $P G, I P, O A, E T, A Z$ and $D M$ were prepared so that the mole fraction of each component was $25 \%$ and equimolar doses of cosolvent donor formulation $(6$ $\mu \mathrm{mol}$ ) were applied to the hairless mouse skin.

In Vitro Percutaneous Penetration - Permeation data were obtained in vitro using vertical diffusion cells with an inside diameter of $25 \mathrm{~mm}$ and receptor compartment filled with $17 \mathrm{ml}$ of $40 \%$ (v/v) PEG 400 in $0.9 \%$ sodium chloride. At selected time intervals the receptor solution was withdrawn and immediately assayed for the drug

\footnotetext{
'Whitby Research, 2801 Reserve Street, Richmond, VA 23261.

'Dow Corning 2020 Willard H. Dow Center Midland, MI 48674.
} 
content by HPLC. Sink conditions were maintained by periodically replacing the receptor fluid and the amount of drug withdrawn was corrected in the calculation of the cumulative amount released. The membrane employed was fresh abdominal female hairless mouse skin (26-28 grams, $8-12$ weeks of age) bred from the progeny of four females and one male SKH-I obtained from Charles River Laboratories. For experiments on stripped skin, the permeability experiments were carried out in the same manner after stripping the abdominal skin 20 times with Scotch tape (3M St Paul, MN) prior to excision. Identification of nifedipine breakdown products and quantitation of nifedipine concentrations were measured by an HPLC method.

Penetration curves were constructed by plotting the cumulative amount of nifedipine, Q, measured in the receptor phase against time. All plots exhibited a linear portion, indicative of steady state penetration, with correlation coefficients greater than 0.90 throughout the linear phase. Lag times were estimated by back extrapolation of the linear portion to the abscissa. When the permeation curve was well defined, linear regression analysis yielded slopes that, when divided by the area $\left(4.9 \mathrm{~cm}^{2}\right)$ yielded the steady state flux value in $\mu \mathrm{g} / \mathrm{h} \cdot \mathrm{cm}^{2}$. An average was computed for each set of replicates.

Mass balance was performed by washing the donor compartments with $10 \mathrm{ml}$ of methanol each, taking care to remove any solid nifedipine particles and to keep the time of contact of methanol to a minimum (less than 3 minutes total). The methanol washes were combined, diluted and measured by HPLC. The receptor phase was changed and the skin was kept in contact for 23 hours with fresh receptor fluid to 
allow any residual drug to leach out. At the end of 23 hours, the receptor phase was removed and analyzed. After another 1 hours of contact between the skin and the fresh receptor phase, the receptor phase was analyzed to assure that no more nifedipine had leached from the skin. No evidence of metabolites was detected and skin binding of drug was low as recoveries of approximately $90 \%$ of the original applied dose were achieved.

Data analysis - Permeability coefficients were determined by dividing the fluxes $\left(\mu \mathrm{g} / \mathrm{h}{ }^{\bullet} \mathrm{cm}^{2}\right)$ by the corresponding solubility of nifedipine in that solvent $\left(\mathrm{mg} / \mathrm{cm}^{3}\right.$ of solution). Mean flux and lag time were compared by ANOVA using Tukey's method at the $95 \%$ level.

\section{RESULTS}

A selection of the physical and laboratory data from the solubility and permeation studies performed on pure alcohols (Table VI) and from donor suspensions of binary cosolvent systems (Table V) are presented. The permeabilities listed were calculated from the linear portion of the $Q$ versus t profile

$$
P=\frac{d Q}{d t} *\left(A * C_{s}\right)
$$

where $\mathrm{P}=$ permeability, $\mathrm{dQ} / \mathrm{dt}=$ the slope of the straight portion of the penetration curve, $\mathrm{A}=$ the surface area $\left(4.9 \mathrm{~cm}^{2}\right)$ and $\mathrm{C}_{\mathrm{s}}=$ the equilibrium saturation concentration of drug in the donor phase.

In Table VII the values of the component proportions, $x_{i}$, were set equal to each other so that the relative effects of the components could be assessed. In many 
Table V. Effects of alkyl chain length on skin permeation of nifedipine from binary mixtures containing alkanoic acids at the $10 \%$ level.

\begin{tabular}{|c|c|c|c|c|c|}
\hline \multicolumn{2}{|r|}{ Vehicle } & $\delta$ & $\mathrm{C}_{\mathrm{s}}$ & $\mathrm{J}$ & $t_{1}$ \\
\hline \multirow{6}{*}{$\begin{array}{l}P \\
E \\
G\end{array}$} & Myristic & 10.4 & 86 & 0 & 24 \\
\hline & Palmitic & 10.5 & 139 & 0 & 24 \\
\hline & Undecylenic & 10.9 & 63 & 0 & 24 \\
\hline & Linoleic & 9.8 & 102 & 11 & 10 \\
\hline & Linolenic & 10.2 & 97 & 3 & 14 \\
\hline & Oleic & 10.3 & 106 & 20 & 10 \\
\hline \multirow[t]{2}{*}{ PG } & Oleic & 13.4 & 8 & 34 & 5 \\
\hline & Undecylenic & 13.6 & 13 & 11 & 4 \\
\hline \multirow{5}{*}{$\begin{array}{l}\mathrm{D} \\
\mathrm{M} \\
\mathrm{I}\end{array}$} & Myristic & 10.8 & 39.6 & 0 & 24 \\
\hline & Palmitic & 9.8 & 82.3 & 0 & 24 \\
\hline & Linoleic & 8.8 & 38.7 & 12 & 1 \\
\hline & Oleic & 9.7 & 34.5 & 15 & 2 \\
\hline & Undecylenic & 9.1 & 24.0 & 0 & 24 \\
\hline
\end{tabular}

KEY: $\delta=$ solubility parameter expressed in Hildebrand units; $\mathrm{C}_{\mathrm{s}}=$ saturation solubility of nifedipine in $\mathrm{mg} / \mathrm{ml} ; \mathrm{J}=$ flux, in units of $\mu \mathrm{g} / \mathrm{h} \cdot \mathrm{cm}^{2} ; \mathrm{t}_{1}=$ lag time in hours (permeation results obtained over the interval $=0$ to 24 hours). Results represent the mean of 3 determinations.

cases, lag times of formulations without hydroxyl moieties were unacceptably long; lag times were found to respond favorably to addition of alcohols. Positive synergistic relationships between combinations of enhancers that modify drug 
Table VI. Effects of alkyl chain length on skin permeability of nifedipine from suspensions of various alcohols at the $10 \%$ level.

\begin{tabular}{||l|c|c|c|c||}
\hline \multicolumn{1}{|c|}{ Vehicle } & $\delta$ & $\mathrm{C}_{\mathrm{s}}$ & $\mathrm{J}$ & $\mathrm{P}$ \\
\hline methanol & 14.5 & 31.2 & 2.2 & 7.1 \\
\hline ethanol & 12.8 & 19.1 & 2.7 & 14.1 \\
\hline butyl & 11.2 & 15.5 & 0.3 & 1.9 \\
\hline isobutyl & 10.3 & 16.0 & 0.5 & 3.1 \\
\hline octanol & 10.3 & 8.1 & 0.6 & 7.4 \\
\hline nonanol & 10.5 & 7.7 & 0.8 & 10.4 \\
\hline decanol & 9.8 & 6.7 & 0.3 & 4.5 \\
\hline dodecanol & 9.1 & 6.0 & 4.2 & 7.0 \\
\hline oleyl & 8.9 & 7.1 & 2.0 & 27.8 \\
\hline
\end{tabular}

KEY: $\delta=$ solubility parameter expressed in Hildebrand units $\llbracket 1$ Hil $=1$ $\left.\left(\mathrm{cal} / \mathrm{cm}^{3}\right)^{1 / 2}\right] ; C_{s}=$ saturation solubility of nifedipine in $\mathrm{mg} / \mathrm{mi} ; \mathrm{I}=\mathrm{flux}$, in units of $\mu \mathrm{g} / \mathrm{h} \cdot \mathrm{cm}^{2} ; \mathrm{t}_{1}=$ lag time in hours (permeation results obtained over the interval $=0$ to 24 hours); $\mathrm{P}=\mathrm{J} / \mathrm{C}_{\mathrm{s}}$. Results represent the mean of 3 determinations (standard deviation in parenthesis).

solubility in skin and those that affect drug diffusivity were shown to affect nifedipine diffusion to improve permeation rates and lag times. In many cases, lag times of formulations without hydroxyl moieties were unacceptably long; lag times were found to respond favorably to addition of alcohols. Positive synergistic relationships between combinations of enhancers that modify drug solubility in skin and those that affect drug diffusivity were shown to affect nifedipine diffusion to achieve desired permeation rates and lag times. 
Table VII. Composition, solubility, and skin permeability for quaternary cosolvent systems prepared by blending PG, IP, OA, AZ, ET, and DM at the $25 \%$ mole fraction level.

\begin{tabular}{|c|c|c|c|c|c|c|c|c|}
\hline \multicolumn{7}{|c|}{ COMPONENT } & \multirow[b]{2}{*}{$\mathrm{C}_{\mathrm{s}}{ }^{1}$} & \multirow[b]{2}{*}{$J^{2}$} \\
\hline CODE & $\begin{array}{l}P \\
G\end{array}$ & $\begin{array}{l}\text { I } \\
\text { P }\end{array}$ & $\begin{array}{l}\mathrm{O} \\
\mathrm{A}\end{array}$ & $\begin{array}{l}A \\
Z\end{array}$ & $\begin{array}{l}\mathrm{E} \\
\mathrm{T}\end{array}$ & $\begin{array}{l}\mathrm{D} \\
\mathrm{M}\end{array}$ & & \\
\hline P5.1 & * & * & * & $*$ & & & 13.0 & $2.3 \pm 3$ \\
\hline P5 2 & & * & $*$ & $*$ & $*$ & & 14.1 & $28.1 \pm 4$ \\
\hline P5.3 & & & * & * & * & * & 34.3 & $6.1 \pm 3$ \\
\hline P5.4 & $*$ & & $*$ & $*$ & * & & 19.3 & $4.5 \pm 1$ \\
\hline P5.5 & & $*$ & & * & * & * & 57.1 & $70.8 \pm 4$ \\
\hline P5.6 & $*$ & & * & & * & * & 19.1 & $120.0 \pm 1$ \\
\hline P5.7 & * & * & & $*$ & & $*$ & & $15.0 \pm 3$ \\
\hline P5.8 & * & * & * & & * & & & $15.1 \pm 2$ \\
\hline P5.9 & & * & $*$ & * & & $*$ & & $2.5 \pm 0.4$ \\
\hline P5.10 & $*$ & & & $*$ & $*$ & $*$ & & $28.1 \pm 3$ \\
\hline P5.11 & $*$ & * & & & * & * & $\mathrm{NA}^{3}$ & $\mathrm{NA}^{3}$ \\
\hline P5. 12 & * & * & * & & & * & 40.2 & $41.8 \pm 3$ \\
\hline P5.13 & $*$ & * & & * & * & & & $69.5 \pm 4$ \\
\hline P5. 14 & & * & * & & * & $*$ & & $5.9 \pm 4$ \\
\hline P5.15 & $*$ & & $*$ & * & & $*$ & & $14.9 \pm 3$ \\
\hline
\end{tabular}

${ }^{1} \mathrm{C}_{\mathrm{s}}=$ nifedipine saturation solubility concentrations expressed as $\mathrm{mg} / \mathrm{ml} .{ }^{2 \mathrm{~J}}=$ flux expressed in units of $\mu \mathrm{g} / \mathrm{h}{ }^{\bullet} \mathrm{cm}^{2}$. ${ }^{3}$ Formulation P5.11 resulted in two immiscible phases. 
The histogram in Figure 11 compares enhancement potential of binary mixtures of equimolar $(10.6 \mu \mathrm{mol})$ amounts of long-chain acid homologs in DMI. Also shown are the effect of addition of Azone at one and ten per cent mole fraction level. There is a clear trend of enhanced nifedipine permeation with increasing chain length in the series of acids and alcohols studied. Nifedipine permeation from dimethylisosorbide suspensions with $10 \%$ oleic acid was 7 fold greater relative to the pure base solvent .

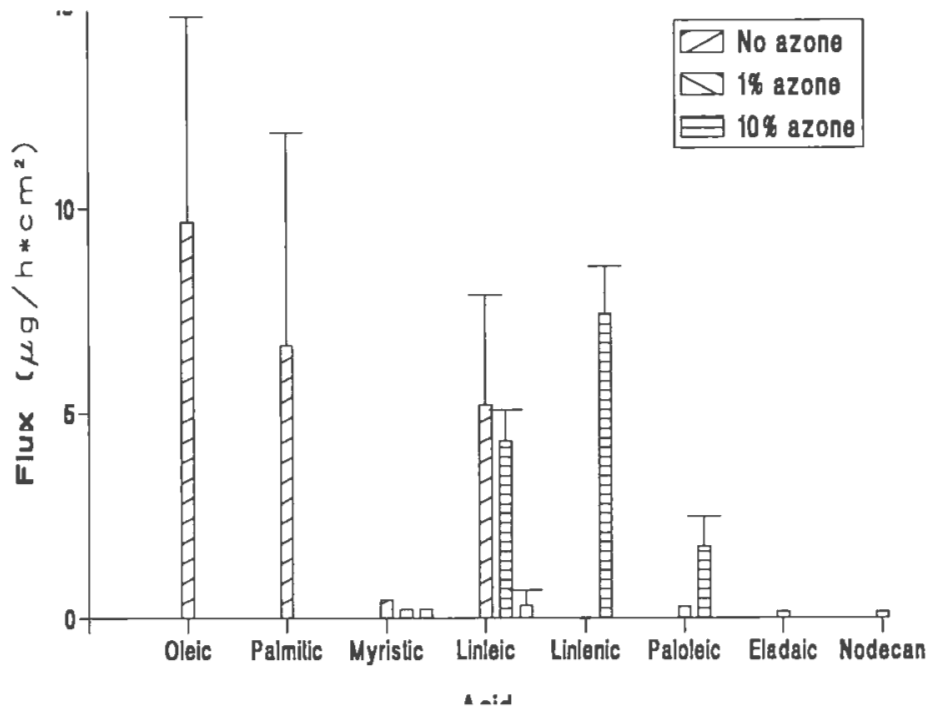

Figure 11. Enhancement potential of binary mixtures of equinolar (10.6 $\mu \mathrm{mol})$ alkanoic acids in DMI. Also shown are the effect of addition of Azone at 1 and $10 \%$ mole fraction level.

Due to the positive effects of cis-oleic acid on the percutaneous adsorption of 
nifedipine noted in Figure 11, this acid was studied more extensively. Figure 12 shows the amount of nifedipine permeated versus time from formulations containing various levels of oleic acid in PEG 400, while the results obtained from oleic acid in DMI-based cosolvent systems are shown in Figure 13. The in vitro diffusion experiments show that oleic acid in combination with DMI or PEG has a positive effect on penetration of nifedipine through hairless mouse skin while DMI or PEG alone were not effective. The results of permeation experiments resulting from suspensions of nifedipine in PEG or DMI containing various concentrations of oleic acid $(1,10,20,30$, and $50 \% \mathrm{w} / \mathrm{w})$ indicate that optimal flux values were obtained with a formulation containing $20 \%-30 \%(w / w)$ oleic acid, enhancing nifedipine absorption by more than 20 fold. Repeated administration of donor at $24-\mathrm{hr}$ confirmed that DMI and PG did not disturb the intrinsic properties of the stratum corneum. Although it readily passes the skin, DMI-based suspensions had poor fluxes over 72 hour study periods. Plots of the amount of drug penetrated versus time revealed that steady state was attained in less than $10 \mathrm{hr}$.

\section{DISCUSSION}

To gain an understanding of the mechanism of skin permeation, the effect of alkyl chain length of alkanols, alkanoic acids, and isopropyl esters on nifedipine skin permeation was investigated. Table VI shows that as the chain length increased, the transdermal permeation rate decreased initially and then increased as the number of methylene groups in the alkyl chain exceeded ten. This effect was also observed for two other lipophilic drugs, indomethacin and progesterone. ${ }^{4}$ The increase in 


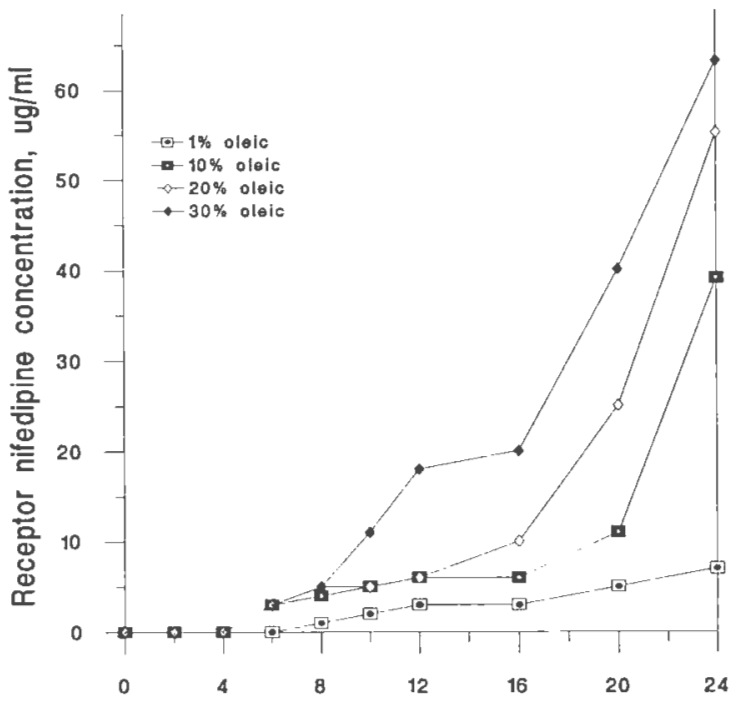

Figure 12 . Effect of cis-oleic acid on nifedipine permeation from polyethylene glycol 400 based donor formulations.

permeation rates observed in donor systems in which the number of methylene groups in the alkyl chain exceeded six to eight has been attributed to their potential to irreversibly alter the barrier function of the skin.

The poor solubility of nifedipine in lipophilic or hydrophilic vehicles makes the selection of an appropriate enhancer difficult. If neither classification truly applies to $\mathrm{N}$, the ideal formulation might include enhancers of disparate actions that act in a positive, synergistic fashion to enhance nifedipine permeation rates. Combinations of enhancers having distinct yet complementary mechanism of actions were conceptualized by Hori. ${ }^{5}$ Aungst et al $^{6}$ showed that the effects of a variety or 


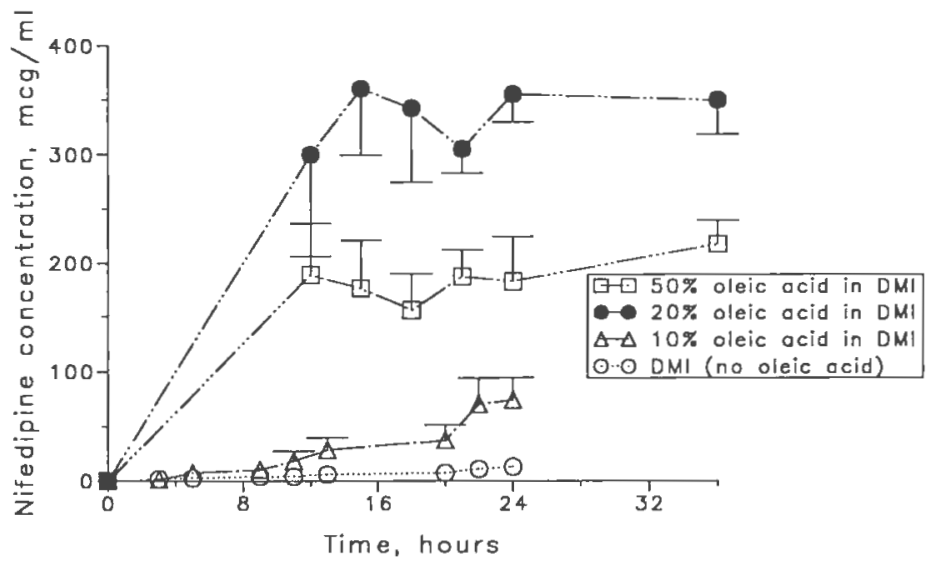

Figure 13. Effect of cis-oleic acid on nifedipine permeation from dimethylisosorbide based donor formulations.

penetration enhancers on naloxone absorption are vehicle dependent. Wotton and coworkers $^{7}$ demonstrated that the choice of vehicle is an important factor and that PG is necessary for promoting the effects of Azone on the permeation of metronidazole; similar effects have been reported for triflurothymidine ${ }^{8}$. Comparison of Figure 12 to Figure 13 shows clearly that the intensity of enhancer effect is highly dependent upon the choice of vehicle.

Azone at levels of $1-3 \%$, has been shown to enhance the penetration of a related dihydropyridine, nicardipine, through excised abdominal skin of the hairless rat using pHEMA as a rate-controlling membrane. ${ }^{9}$ Previous work has shown that the optimum concentration of Azone varies with both drug and the formulation being 
examined with $2-10 \%$ enhancing while excessive amounts having deleterious effects on permeation. ${ }^{10}$ Sezaki et al $^{11}$ observed that as the oil:water partition coefficient increases, there is a tendency for penetration to decrease in skin not pretreated with Azone and Azone-like enhancers and noted that a time element may be involved. The response of nifedipine permeation to Azone is shown in Figure 14. The permeation of nifedipine under the influence of oleic acid and Azone are quite different, suggesting that there are distinct loci in the stratum corneum upon which they exert their effect. Neither Azone ${ }^{12}$ or $\mathrm{PEG}^{13}$ is systemically absorbed to appreciable degree. Although both materials are very good solvents for nifedipine, azone, like PEG, had little effect on skin permeability as seen in Figure 14. When DMI or PG are substituted for PEG as the base solvent in the binary mixtures, permeation rate is magnified several fold. Since DMI and PEG share similar solubilities for nifedipine, the enhancement cannot be attributed to enhancement of thermodynamic activity. Both DMI and PG are readily absorbed when applied topically, suggesting that higher flux values may be linked to the ability of the solvent to penetrate and modify the dynamics of drug:skin partitioning.

Experiments on stripped skin have shown that a significant barrier to diffusion still exists even when the stratum comeum has been removed. Figure 15 is particularly interesting in that stripped skin offered slightly less resistance to nifedipine diffusion than intact skin. Similar results have been observed for DMSO, ${ }^{14}$ which apparently swells the corneocytes, reducing their barrier qualities. Other possible mechanisms include partial extraction of lipids and protein configurational 


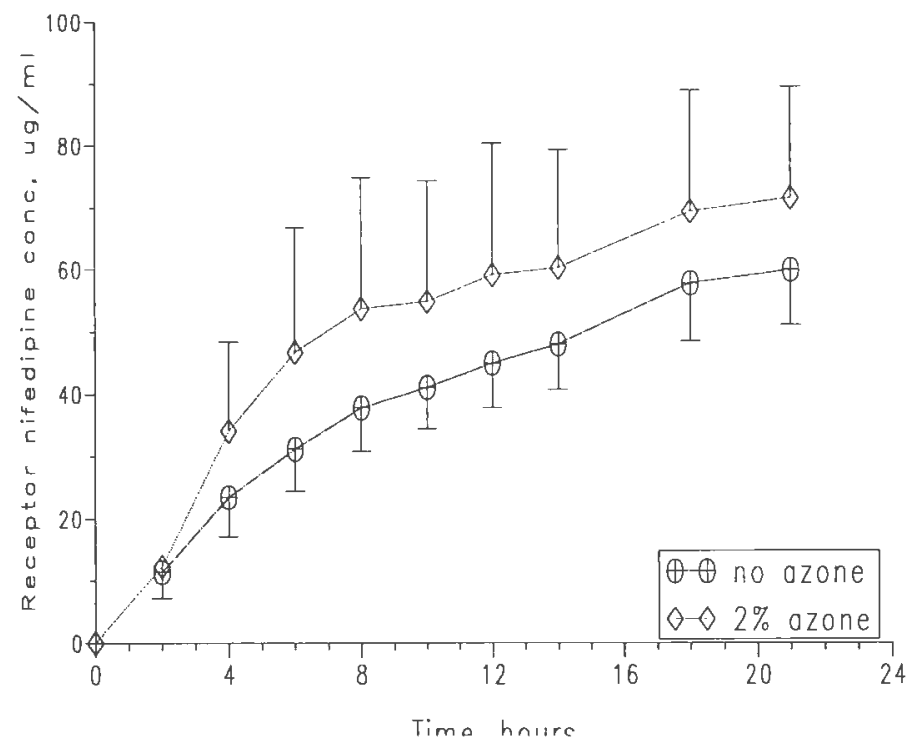

Figure 14. Effect of $2 \%$ Azone on nifedipine permeation from IP:OA:ET:PG suspensions $(n=3)$.

changes resulting from replacement of water by the vehicle. ${ }^{15}$ Lipophilic solutes which permeate readily through the stratum corneum may be rate-limited by the epidermal or dermal layers. In the case of nifedipine, removal of the stratum corneum lipids probably accelerates the movement of the PG, ET and DM components which results in more rapid depletion of these key components from the donor phase. In this way, the results of the skin stripping experiment lend further credence to cosolvent control.

The data obtained suggest strongly that enhanced transport is dependent on 
several factors. Table VIII lists, in a qualitative manner, the relative degree of effect on the stratum corneum, degree of systemic absorption, and relative solubility for nifedipine of various vehicles and their resultant fluxes. When viewed in the context of the skin stripping experiments, the observations represented in Table VIII show maximum flux values are obtained from mixtures that both disrupt the stratum corneum and create conditions that favor partitioning of drug from the epidermal into the dermal layers. It is interesting to speculate on the source of the difference in shape of the Q vs t curves from DM vs PEG based binary formulations. While DM and PEG possess comparable $\mathrm{C}_{\mathrm{s}}$ values, the former readily permeates the skin while the latter does not. Since the donor was applied as a suspension (an infinite dose design), the asymptotic behavior of the profiles in Figure 13 are unexpected while similar experiments, using PEG as the base solvent displayed in Figure 12, do not. The rapid depletion of the DM component from the donor would explain why $Q$ versus t profiles for systems containing small amounts of DM display asymptotes well before supply of nifedipine in the donor system is exhausted and constitute strong evidence for a possible linkage of solvent:drug in the permeation process.

For a very lipid-soluble drug, clearance from the viable tissue may replace diffusion through the stratum corneum as the rate limiting step in the overall process of percutaneous absorption. Conventional models portray the skin beneath the patch as a sink for the permeating molecule. If this assumption does not hold, passage through the stratum corneum may no longer be the rate limiting step to permeation and it would be incorrect to target the stratum corneum as the barrier to diffusion. In 
Table VIII . Qualitative predictors of nifedipine permeation.

\begin{tabular}{||c|c|c|c|c||}
\hline Vehicle & $\mathrm{C}_{\mathrm{s}}$ & PEA & Passage & $\mathrm{J}$ \\
\hline PEG & High & 0 & 0 & 0 \\
\hline DMI & High & 0 & High & 0 \\
\hline OA & 0 & High & 0 & 0 \\
\hline PG & 0 & High & High & 0 \\
\hline Azone & High & High & 0 & 0 \\
\hline OA:DMI & High & High & High & High \\
\hline
\end{tabular}

KEY: $\mathrm{C}_{\mathrm{s}}=$ saturation solubility; $\mathrm{PEA}=$ penetration enhancement activity; Passage $=$ permeability of vehicle; $\mathbf{J}=$ flux.

such cases, patches that incorporate penetration enhancers whose actions are restricted to altering the barrier properties of the stratum corneum would be inferior to those that incorporate agents that would promote partitioning from the epidermal to the dermal layers.

The partition coefficient is crucially important in promoting a high initial concentration of the agent within the upper strata of the epidermis. Based on the solubility parameter of $10\left(\mathrm{cal} / \mathrm{cm}^{3}\right)^{1 / 2}$ for porcine skin, one would expect nifedipine to have a high affinity for the stratum corneum. Scheuplein has calculated that a tissue-water partition coefficient of $>400$ was required before a 200 micron layer of dermis had a diffusional resistance comparable to that of stratum corneum 10 microns thick. ${ }^{16}$ With an octanol-water partition coefficient of $10,000: 1$, a significant 
resistance to nifedipine may well reside in the deeper dermal layers.

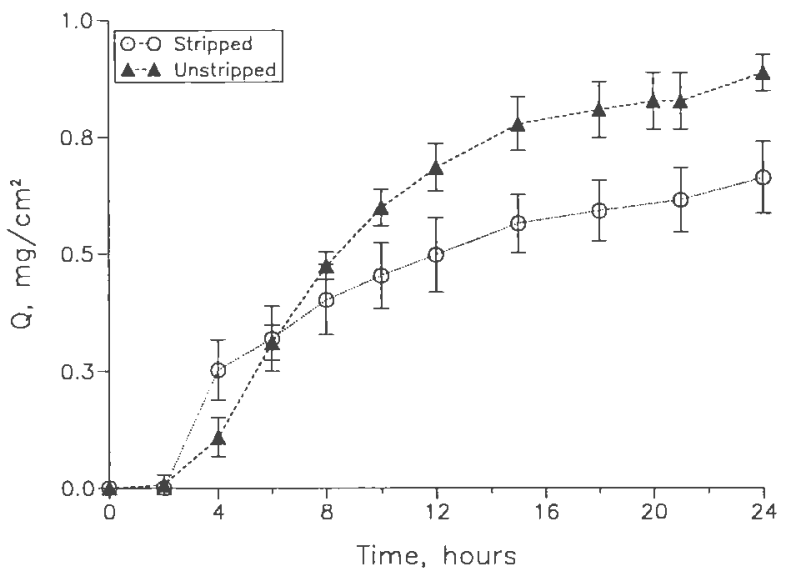

Figure 15. Optimization of PG:OA:ET:DM formulations based on the ridge analysis in Table XV: Permeation of nifedipine from formulation P42.1 in intact and stripped skin.

Notice in Tabie $V$ that in a binary systems, with oleic acid added to an interacting (DM) or a non-interacting (PE) solvent with similar saturation solubilities for nifedipine, there is essentially very little difference in nifedipine permeation. However, when binary systems of PG and OA are compared to the DM and OA systems, the results are quite different. That is, systems of PG and OA (which have low $\mathrm{C}_{\mathrm{s}}$-values) promoted flux to a much greater degree with shorter lag times than corresponding DM:OA systems of higher nifedipine saturation solubility. 
Furthermore, although Table $\mathrm{V}$ and Table VII show poor results with saturated acids and alcohols with molecular weight near that of myristic acid, the second and third highest $\mathbf{J}$ values derive from formulations containing the isopropyl ester of myristic acid. IPM, like PG, did not solubilize nifedipine as well as DMI or PG, yet higher fluxes were achieved from these systems. This is probably due to the fact that nifedipine, being more soluble in the DM:OA cosolvent, prefers to remain in the donor compartment.

Another plausible explanation is that PG, being much more polar than DM, enhances the movement of OA out of the donor, effectively "pushing" the OA into the skin where its effect can be exerted. This push effect is augmented by the effect of transpired moisture. Solubility studies in ternary mixtures of PEG:DMI:water and PG:DMI:water showed that the solubility of nifedipine is extremely sensitive to the presence of water. Because of the very low aqueous solubility of nifedipine, as the skin becomes hydrated and the donor acquires moisture the donor may form supersaturated solutions. Transpired moisture absorption, when calculated on basis of mole fraction, has a significant effect on raising the solubility parameter of the donor. Essentially, donors that cannot interact and sequester water molecules (higher alcohols and acids) should and do display a larger "push" effect.

The asymptotic behavior of the $\mathrm{Q}$ versus t curves at $12-24 \mathrm{~h}$ for some cosolvent blends deserves comment. All factors being equal, the maximal rate of transdermal transport should occur from systems of higher thermodynamic activity, that is from suspensions versus solutions. In Table IX the results obtained from a system 
containing drug at $50 \%$ below and $10 \%$ above its experimentally determined saturation solubility show suspension and solution gave comparable permeation rates.

Table IX. Effect of drug level on permeation: comparison of suspension versus solution."

\begin{tabular}{|c|c|c|c|c|c|c|}
\hline \multicolumn{5}{|c|}{ Mole fraction percent } & \multirow[b]{2}{*}{$\mathrm{C}_{\mathrm{s}}^{\mathrm{b}}$} & \multirow[b]{2}{*}{$\mathbf{J}^{\mathbf{c}}$} \\
\hline PG & IP & $\mathrm{AZ}$ & ET & DM & & \\
\hline 19 & 5 & 3 & 16 & 58 & - & $52.2 \pm 12.6$ \\
\hline 19 & 5 & 3 & 16 & 58 & 85.3 & $48.1 \pm 11.4$ \\
\hline 27 & 8 & 4 & 22 & 41 & - & $121.8 \pm 16.2$ \\
\hline 27 & 8 & 4 & 22 & 41 & 90.5 & $100.3 \pm 18.4$ \\
\hline
\end{tabular}

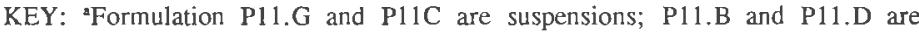
solutions (50\% of saturation solubility). ${ }^{b}$ Concentration values are expressed as $\mathrm{mg} / \mathrm{ml}$. 'Flux values are expressed in units of $\mu \mathrm{g} / \mathrm{h} \bullet \mathrm{cm}^{2}$.

If saturated and subsaturated mixtures represent different levels of activity, these results show that thermodynamic activity is not a critical factor in these systems. If excess nifedipine is present and sink conditions are maintained by replenishment of receptor, what may account for the abrupt decrease in permeation? A plausible explanation is that the supply of one or more component of the cosolvent mixture has become exhausted. (Figure 18 on page 108 shows the curve shape following application of increasing donor volumes; although no significant difference in the slopes are discernable, the duration of steady state flux is clearly greater when the number of moles of cosolvent molecules is trebled) The permeation of hydrophobic 
drugs such as nifedipine may be contingent upon the concomitant permeation of cosolvent. It might be necessary to incorporate significant amounts of cosolvent in the patch in order to maintain desired permeation rates. Thus, the previous discussion regarding the dependence of the shape of the $Q$ versus t profiles to the amount of DM in the formulation is entirely consistent with our earlier hypothesis that the diffusion of nifedipine is inextricably linked to the codiffusion of solvent

Extraction of hairless mouse skin following permeation experiments in vitro recover very small quantities $(<10 \%)$ of the administered dose. Two inferences emerge from this observation: either the skin as a whole has poor affinity for nifedipine (i.e., the solubility parameter is not $10 \mathrm{Hil}$ ) or that the deposition of nifedipine in the SC is minimal (i.e., the skin does indeed have a high binding potential for nifedipine, but that it constitutes such a small percentage of the skin that the quantity of nifedipine that it contains is exceedingly small). Alternatively, the solvent may dissolve the stratum corneum, leaving skin with very different qualities. This latter alternative is unlikely, since J values obtained post 24 hours following repeat application of donor were similar to those obtained from the steady-state portion of the $0-24$ hours $Q$ versus $t$ profiles.

The data in Table VII summarizes the intrinsic activity on nifedipine permeation enhancement from the 15 possible quaternary blends. Clearly, formulations without DM showed very poor permeation rate compared with those with DM present $(\mathrm{p}<0.05)$. Mixtures pairing PG with DM (e.g., P5.7) displayed enhancement factors five times greater than those that did not. While $A Z$ appears to 
have little effect in formulations P5.1 (PG:IP:OA:AZ) and P5.14 (PG:IP:AZ:ET), formulations combining AZ with DM e.g., P5.7 (PG:IP:AZ:DM) and P5.10 (PG:AZ:ET:DM) showed enhancement activity, suggesting that DM might exert its effects by enhancing the activity of other enhancers. Mixtures of ET, PG and DM displayed overall the highest enhancement factors. The synergistic relationship among these three components was highest in the presence of OA (P5.6 and P5.13). A maximum flux of was $120.2( \pm 10.3) \mathrm{ug} / \mathrm{h} \cdot \mathrm{cm}^{2}$ was observed from formulation P5.6 consisting of PG:OA:ET:DM, each at the $25 \%$ level. In a separate set of experiments, the solvents listed in Table VII were applied individually as nifedipine suspensions to the skin. The $Q$ versus $T$ profiles obtained following application of nifedipine suspended in pure ET, IP, OA, PG, or DM showed that none individually possessed the necessary intrinsic activity to match the synergistic effects observed in mixtures of these solvents, particularly the 25:25:25:25 PG:OA:ET:DM quatemary mixture. These results suggest that a ternary and possibly quaternary mixture of PG, $\mathrm{OA}, \mathrm{ET}$ or $\mathrm{DM}$ is required to achieve optimum fluxes from formulations employing these components.

Our interpretation of these results is as follows. Given that $\delta_{\text {nif }} \approx \delta_{\mathrm{sc}}$, binary mixtures whose solubility parameter approaches that of the stratum corneum have higher $\mathrm{C}_{\mathrm{s}}$ values and $\mathrm{J}$ values should decrease. Conversely mixtures having solubility parameters greater than 10 have lower nifedipine solubilities, a condition which favors drug movement from donor into skin. This trend is observed in Table VI and Table VII, where $\delta$ values are associated with higher J values. Therefore, the ideal 
patch design would encompass the following functions: (1) initial high activity of drug in the patch creates a severe gradient forcing the drug from the patch into the epidermis; (2) release of enhancer(s) to alter the diffusion coefficient of the drug in the stratum corneum; (3) release of cosolvent at a prescribed rate so as to modify nifedipine solubility in the lower epidermal and dermal layers.

Conclusions - Unsaturated, long-chain acids had pronounced effects which appear to be selective for cis-unsaturated fatty acids as they were not observed for their saturated ( myristic, and nonadecanoic) or trans-unsaturated acids (elaidic) counterparts. It was found that the permeability of nifedipine through hairless mouse skin is controlled by both the solubility of the drug in the donor and the ability of donor to both disturb and pass through the epidermal skin layers. The shape of $Q$ vs $t$ plots displayed a strong dependency on the quantity of cosolvent applied. These results imply that a linkage between nifedipine and vehicle codiffusion may exist and further studies to establish the nature of this dependency are presently underway. 


\section{REFERENCES}

1. Guy, R.H.; Hadgraft, J. "Transdermal Drug Delivery: The Ground Rules are Emerging." Pharm.Intl., 1985, 6, 112-116.

2. Squillante, E. T. Needham, and H. Zia. Document in preparation.

3. Snee, R.D.; Marquardt, D.W. "Extreme vertices designs for linear mixture models." Technometrics, 1974, 16, 399-408.

4. Chien, Yie W.; Xu, H; Chiang, C.C.; Huang, Y.C. "Transdermal Controlled Administration of Indomethacin. I. Enhancement of Skin Permeability." Pharm. Res. 1988, 5, 103-106.

5. Hori, M.; Maibach, H.; Guy, R. "Enhancement of Propranolol and Diazepam Skin Absorption In Vitro. II: Drug, Vehicle, and Enhancer Penetration Kinetics." J.Pharm. Sci. 1992, 81(4), 330-333.

6. Aungst, B.J.; Rogers, N.J; Shefter, E. "Enhancement of Naloxone Penetration Through Human Skin In Vitro Using Fatty Acids, Fatty Alcohols, Surfactants, Sulfoxides and Amides." Int. J. Pharm. 1986, 33, 225-234.

7. Wotton, P.K.; Mollgaard, B; Hadgraft, J; Hoelgaard, A. "Vehicle Effect on Topical Drug Delivery. III. Effect of Azone on the Cutaneous Permeation of Metronidazole and Propylene Glycol." Int. J. Pharm. 1985, 24, 19-26.

8. Sheth, N.V.; Freeman, D.J.; Higuchi, W.I.; Spruance, S.L. "The Influence of Azone, Propylene Glycol and Polyethylene Glycol on In Vitro Skin Penetration of Triflurothymidine." Int. J. Pharm. 1986, 28, 201-209.

9. Toshinobu, S. "Percutaneous Absorption Enhancer Applied to Membrane Permeation Controlled Transdermal Delivery of Nicardipine $\mathrm{HCl}$." Drug Design and Delivery, 1989, 4, 69-75.

10. Stoughton, R.; McClure, W.O. "Azone: A New Non-Toxic Enhancer of Cutaneous Penetration." D.D.I.P. 1983, 9, 725-744.

11. Okamoto, H.; Hashida, M.; Sezaki, H. "Effect of 1-Alkyl or 1.Alkylazacycloalkanone Derivative on the Penetration of Drugs with Different Lipophilicities through Guinea Pig Skin." J.Pharm.Sci 1991, 80, 41-45.

12. Wiechers, J.W.; De Zeeuw, R.A. "Transdermal Drug Delivery: Efficay and Potential Applications of the Penetration Enhancer Azone." Drug Design and Delivery, 1990, 6, 87-100. 
13. Ruddy, S.B.; Hadzija, B.W. Influence of Molecular Size on the Iontophoretic Transport of Polymeric Nonelectrolytes Through Hairless Rat Skin. Paper presented at the GRASP Eastem Regional Meeting, Durham, N.C. 23 May 1991.

14. Yu, C.D. J.Pharm.Sci 1980, 69, 770-772.

15. Oertel, R.P. "Protein Conformation Changes Induced in Human Stratum Comeum by Organic Sulfoxides: an IRS Investigation." Biopolymers 1977, 16, 2329-2345.

16. Scheuplein, Robert J. In A. Jarrett, ed. The Physiology and Pathophysiology of the Skin New York: Academic Press 1978 1693-1730. 
FORMULATION OPTIMIZATION 


\begin{abstract}
The effects of transdermal device components on nifedipine permeation were evaluated by (1) identifying those factors which significantly affect the permeation of nifedipine and (2) selecting levels for these factors which optimize flux and lag times. Of primary interest was the role of solubility and its influence on the permeation enhancers chosen to increase the skin permeability of this hydrophobic calcium channel blocker. A McLean Anderson mixture design was employed to obtain a stoichiometric interpretation of the permeation process. In addition to demonstrating solvent-induced permeation enhancement, the mechanisms by which solvents enhance permeation are discussed. The ultimate objective was to apply regression techniques to interpret, on a molar basis, the data obtained from in vitro permeation experiments. The model obtained was used to identify both the magnitude and significance of the synergism between formulation components and to optimize the desired response. Data from these inquiries have contributed toward the rational selection of components in a viable transdermal dosage form that permit transdermal delivery of nifedipine at rates in excess of the $31 \mu \mathrm{g} / \mathrm{h} \bullet \mathrm{cm}^{2}$ required for potential therapeutic response.
\end{abstract}




\section{Introduction}

Propylene glycol (PG), ethanol (ET) and dimethylisosorbide (DM) are known to be well absorbed when administered topically. The results of previous experiments infer that the skin permeability of nifedipine is related to the volume of donor cosolvent applied, suggesting that a reservoir-type device would be best suited to deliver drug and enhancer to the skin. The results obtained from differential scanning calorimetry show nifedipine to have strong intermolecular attractions, leading to the hypothesis that solubilization of drug may provide a means of increasing the amount of nifedipine molecules available for diffusion. Information obtained from previous solubility studies indicate that movement of nifedipine from the epidermis to an environment of extensive hydrogen bonding characteristic of the hydrated dermis is likely to lower the driving force of diffusion. Thus, formulation components were purposely selected for their potential to solubilize and facilitate nifedipine diffusion across the epidermis and dermis.

Visualization of the permeation process as an intimate relationship between a finite ratio of formulation components may offer clues into the mechanism of the transdermal diffusion process. Inherent in the stoichiometric approach is that a change in one component requires collateral change in the next; a deficiency best handled by the mixture experimental designs. It is difficult to interpret the results of experiments involving mixtures of more than three components. This partly due to the inability to visualize spatially the effect of a change in a dependent variable on the measured response. In addition, any change in proportion of one component must be 
coupled to changes elsewhere in the composition of the mixture, rendering a superficial interpretation of the response quite meaningless. These two qualities pose an intractable problem best solved by several published mixture experimental designs.' A distinguishing feature of these designs is that the independent, controllable factors represent non-negative, proportionate amounts of the mixture components rather than unrestrained amounts which must of course sum to unity.

The problems associated with identifying a blend of formulation components which would allow adequate permeability with minimal irritancy and lag time at a reasonable cost, may be solved using mixture response surface methodology. Typically the effects of various factors on certain characteristics of a system are studied, with an eye toward: (1) identifying those factors which significantly affect permeation, and (2) selecting levels for these factors which optimize the permeation characteristics. The first step involves the statistical problem of fitting a model to experimental data to disclose which factor level combinations are critical or not necessary to control. The second step, for the model disclosed in the first step, involves finding the combination of the factor levels that either minimize or maximize the response. Although the factors are observed only at a finite number of values, the factors are treated as continuous variables. Using the ranges of each factor, we interpolate over their composite range in order to optimize the responses. We employ various facilities available in the SAS system in conjunction with a modified McLean and Anderson experimental design with the objective of optimizing levels of formulation components which maximize flux yet minimize lag time. 


\section{METHODS}

Kinetic studies - The in vitro release of nifedipine was investigated by applying cosolvent mixtures containing $10 \%$ excess suspended nifedipine to freshly excised hairless mouse skin mounted in vertical Franz cells. Equimolar amounts (10 $\mu$ mol of cosolvent) of the various donor formulations are applied to all cells and the experiment conducted as described previously. ${ }^{2}$ We adopt the convention of expressing the levels of the factors (i.e., the relative amounts of formulation components) in terms of mole fraction. The responses of interest are the lag time and steady state flux and represent the average values taken over 4 replicates of each formulation. Samples of the receptor phases $(3 \mathrm{ml})$ were removed, filtered through 0.45 micron filters and $1 \mathrm{ml}$ reserved for HPLC analysis. Serial sampling was generally performed at $2,4,6,8,10,12,15,18,21$ and 24 hours after the suspensions were applied. The samples of receptor fluid were placed in amber vials, labelled and immediately analyzed. A stability-indicating HPLC method was employed to measure skin permeation kinetics.

Data analysis - The experimental design, designated Design I, constitutes a polyhedron whose centroids are established by a two-step procedure described by McLean and Anderson. ${ }^{3}$ Mixtures of propylene glycol $\left(\mathrm{x}_{1}\right)$, oleic acid $\left(\mathrm{x}_{2}\right)$, ethanol $\left(x_{3}\right)$ and dimethylisosorbide $\left(x_{4}\right)$ were prepared in a manner such that their proportions are non-negative, must sum to unity and are restricted to the following upper and lower bounds:

$$
10 \%<x_{1}<72 \%
$$




$$
\begin{aligned}
& 8 \%<x_{2}<25 \% \\
& 10 \%<x_{3}<25 \% \\
& 10 \%<x_{4}<70 \%
\end{aligned}
$$

The design points comprise the vertices and centroids of a convex polyhedron suitable for fitting first-degree and quadratic models in the constrained mixture space. The resulting factor space and the design point designations are presented in Figure 16. Insight gained from this design permitted tighter constraints on the experimental space such that the mole fraction of $\mathrm{OA}$ and ET was fixed at the $10 \%$ level and the levels of the other two components were allowed to vary. This second experiment is referred to as Design II. Experimental design, analysis and optimization were performed using the ADX ${ }^{\mathrm{TM}}$ menu system of SAS/QC and SAS/STAT software, Version 6.0 (SAS Institute, Inc. Carey, NC).

\section{Results and Discussion}

Of the many factors that will influence the effectiveness of penetration enhancers, this study focuses on the physicochemical properties of the permeant and vehicle with the objective of relating, on a molecular level, how the four components affect both responses of flux and lag time. It is unlikely that for this multi-response problem there exists a unique combination of factors which result in optimum values for both responses simultaneously. Instead, we first identify the different sets of factors affecting the different responses. Once these two sets are defined, a compromise is accepted by optimizing flux while holding the lag time under a certain level. The data in Table $\mathrm{X}$ represent permeation measurements obtained from Design 
I. While the maximum flux in Table $\mathrm{X}$ is attained at high values of oleic acid, reasonable flux values are also observed for low values of this factor. Acceptable flux and lag times were obtained from systems containing levels of oleic acid far below its upper constraint of $25 \%$. Likewise, Table XI shows that better fluxes are obtained with lower (10-20\%) levels of DM. Also, since OA is the more chemically labile, irritating to the skin and expensive than any of the other factors, it is important to limit the amount of this critical component in the proposed transdermal delivery system.

The results which appear in Table $\mathrm{X}$ and Table XI offer the following observations: (1) quaternary mixtures using OA and ET both at the $10 \%$ level offer significant enhancement of flux values; (2) lag times were clearly longer for formulations containing levels of DM exceeding 20-30\%; (3) both lag time and flux are improved by limiting the level of DM to less than $20 \%$. These results suggest that a relatively high level of $\mathrm{PG}$ and low levels of OA, ET and DM will give maximum flux and minimum lag times. A possible interpretation of these observations is as follows. Although the disposition of DM following topical administration is unresolved, it is a universal solvent believed to readily pass the epidermis. ${ }^{4}$ Curiously, the single solvent solubility of nifedipine is highest in DM $(85 \mathrm{mg} / \mathrm{ml})$ than in OA $(0, \mathrm{~m} / \mathrm{ml})$, ethanol $(17 \mathrm{mg} / \mathrm{ml})$ or PG $(14 \mathrm{mg} / \mathrm{ml})$. Previous solubility studies revealed that nifedipine solubility in binary mixtures of PG and DM drop precipitously as small amounts of water are added to form ternary PG:DM:water mixtures. Given its very low aqueous solubility $(\sim 0.006 \mathrm{mg} / \mathrm{ml})$ nifedipine would 


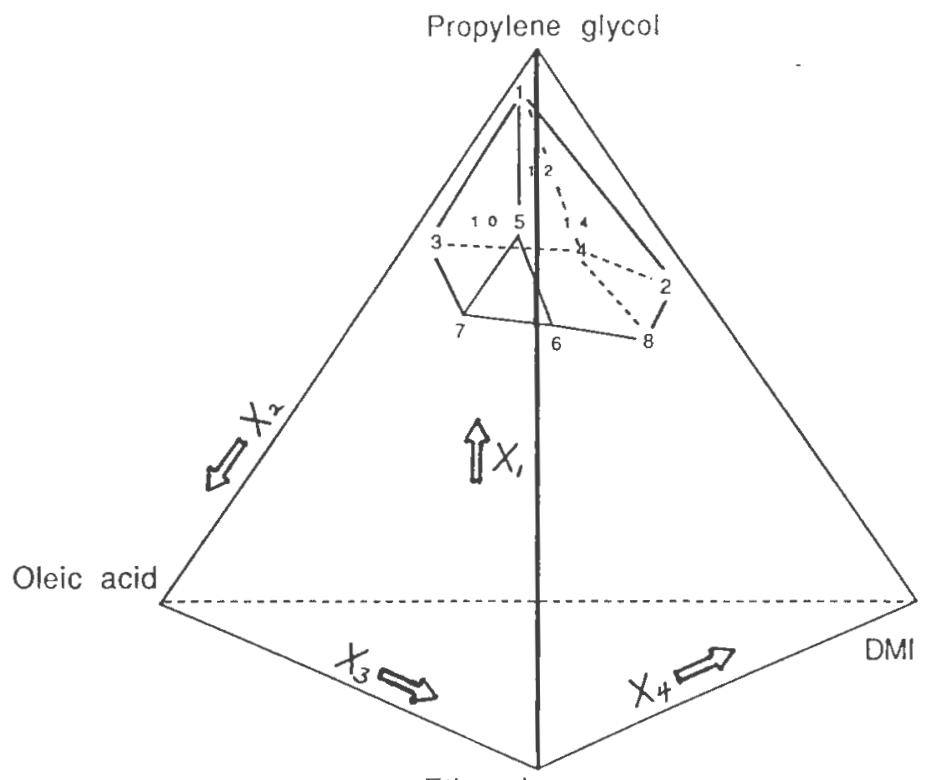

Ethanol

Figure 16. Experimental sample space for the four component McLean Anderson mixture design $\left(\mathrm{X}_{1}=\mathrm{PG} ; \mathrm{X}_{2}=\mathrm{OA} ; \mathrm{X}_{3}=\mathrm{ET} ; \mathrm{X}_{4}=\mathrm{DM}\right)$. 
Table X. Design I. Quaternary systems data for a four-component simplex-centroid design: PG, OA, ET, and DM for donor suspensions using the following constraints: $\mathrm{PG}=10-75, \mathrm{OA}=10-36, \mathrm{ET}=10-36, \mathrm{DM}=10-75$.

\begin{tabular}{||c|c|c|c|c|c|c||}
\hline \multirow{2}{*}{$\mathrm{N}$} & \multicolumn{3}{|c|}{ Mole Fraction Percent } & \multicolumn{2}{c||}{ Response Variables } \\
\cline { 2 - 7 } & PG & OA & ET & DM & $\begin{array}{c}\text { Lag time } \\
\text { (hours) }\end{array}$ & $\begin{array}{c}\text { J } \\
\left(\mathrm{ug} / \mathrm{h} \cdot \mathrm{cm}^{2}\right)\end{array}$ \\
\hline \hline 1 & 72 & 10 & 8 & 10 & $2.4 \pm 1.0$ & $148.9 \pm 60.6$ \\
\hline 2 & 55 & 25 & 10 & 10 & $2.0 \pm 2.2$ & $60.0 \pm 25.3$ \\
\hline 3 & 55 & 10 & 25 & 10 & $1.9 \pm 5.7$ & $96.0 \pm 53.1$ \\
\hline 4 & 10 & 10 & 25 & 55 & $3.3 \pm 0.8$ & $12.2 \pm 2.4$ \\
\hline 5 & 10 & 10 & 10 & 70 & $4.8 \pm 0.3$ & $11.0 \pm 0.8$ \\
\hline 6 & 25 & 10 & 10 & 55 & $3.7 \pm 0.4$ & $58.8 \pm 45.7$ \\
\hline 7 & 14 & 36 & 36 & 14 & $3.8 \pm 0.6$ & $48.2 \pm 7.5$ \\
\hline 8 & 10 & 25 & 25 & 40 & $7.6 \pm 1.0$ & $48.2 \pm 5.5$ \\
\hline 9 & 25 & 25 & 25 & 25 & $2.5 \pm 1.4$ & $120.2 \pm 10.3$ \\
\hline
\end{tabular}


Table XI. Design II. Four-component simplex-centroid blending propylene glycol (PG), cis-oleic acid (OA), ethanol (ET), and dimethylisosorbide (DM) for donor suspensions keeping $[\mathrm{OA}]$ and $[\mathrm{ET}] \leq 10 \%$.

\begin{tabular}{|c|c|c|c|c|c|c|}
\hline \multirow[b]{2}{*}{$\mathbf{N}$} & \multicolumn{4}{|c|}{ Mole Fraction Percent } & \multirow{2}{*}{$\begin{array}{c}\text { Iag } \\
\text { Time } \\
\text { (hours) }\end{array}$} & \multirow{2}{*}{$\underset{\left(\mathrm{ug} / \mathrm{h} \cdot \mathrm{cm}^{2}\right)}{\mathrm{J}}$} \\
\hline & $P G$ & OA & ET & DM & & \\
\hline 1 & 97 & 1 & 1 & 1 & $3.8 \pm 0.6$ & $110 \pm 16$ \\
\hline 2 & 88 & 1 & 1 & 10 & $7.6 \pm 1.0$ & $106 \pm 25$ \\
\hline 3 & 88 & 10 & 1 & 1 & $2.0 \pm 0.5$ & $60 \pm 30$ \\
\hline 4 & 79 & 10 & 1 & 10 & $2.3 \pm 0.3$ & $117 \pm 10$ \\
\hline 5 & 88 & 1 & 10 & 1 & $5.1 \pm 0.7$ & $80 \pm 19$ \\
\hline 6 & 79 & 1 & 10 & 10 & $4.8 \pm 0.4$ & $103 \pm 10$ \\
\hline 7 & 79 & 10 & 10 & 1 & $0.7 \pm 0.4$ & $101 \pm 2$ \\
\hline 8 & 70 & 10 & 10 & 10 & $2.4 \pm 1.0$ & $149 \pm 6$ \\
\hline 9 & 82 & 6 & 6 & 6 & $3.3 \pm 1.3$ & $104 \pm 46$ \\
\hline 10 & 88 & 6 & 6 & 1 & 3 & 131 \\
\hline 11 & 79 & 6 & 6 & 10 & 2 & 111 \\
\hline 12 & 88 & 6 & 1 & 6 & 3 & 109 \\
\hline 13 & 79 & 6 & 10 & 6 & 2 & 125 \\
\hline 14 & 88 & 1 & 6 & 6 & 3 & 99 \\
\hline 15 & 79 & 10 & 6 & 6 & 2 & 127 \\
\hline
\end{tabular}

KEY: Design points, N, $1-8$ represent vertices; point 9 represents the overall centroid; points $10-15$ represent face centroids. $J$ is the steady state flux in $\mathrm{ug} / \mathrm{h} \bullet \mathrm{cm}^{2}$ reported as the mean \pm s.d. of 4 cells except points $10-15$ which were single determinations.. 
have little affinity for the hydrated epidermis and consequently the diffusional driving force from the donor into the dermal layers is likely to be lower. A possible explanation why poorer fluxes and shorter lag times were obtained from those formulations containing only small quantities of DM is that although DM is a good solvent and effectively increases the amount of nifedipine readily available in solution, donors with high fractions of DM may impede nifedipine permeation by altering nifedipine partitioning in favor of the donor phase. This effect would be expected to dissipate with time as DM levels fall in the donor. If this were true, donors with greater mole fraction DM would display longer lag times.

To test this theory, Design II was augmented with additional formulations such that the ratio of OA to ET was held constant and the proportion of PG to DM allowed to vary. These results, reported in Table XII, show a monotonic decrease in lag time as the level of DM falls. For this series of experiments a clear demarkation was observed in the character of the $Q$ versus $t$ profiles as the ratio of PG to DM varied. A portion of the data, represented in Figure 17, shows the disparate nature of the curves. Donor formulations with DM levels exceeding $20 \%$ consistently displayed lag times of 10 hours or more which were significantly different from the lag times observed in donors with less than I0\% DM. Formulations containing less than $30-40 \%$ DM display asymptotic behavior with the $Q$ versus $t$ curve leveling out at around 18 hours; this behavior was not observed for formulations whose DM content exceeded $50 \%$. Interestingly, formulations containing 30 to $60 \%$ DM did not demonstrate this asymptotic leveling but exhibited biphasic $Q$ versus $T$ profiles, 
making the determination of lag time extremely subjective. For the biphasic profiles, since the latter portion of the profile might possibly represent chemical damage to the skin induced by donor formulation, the lag time and flux values were obtained from the initial portion of the curves. Asymptotic and biphasic $Q$ versus $T$ profiles were not observed in formulations in which the level of DM exceeds $70 \%$. The permeation rates displayed by these formulations were so low that they are indistinguishable from the abscissa in Figure 17.

Table XII. Quaternary systems data for a four-component blending propylene glycol (PG), cis-oleic acid (OA), ethanol (ET), and dimethylisosorbide (DM) donor suspensions keeping OA:ET ratio constrained at 10:10.

\begin{tabular}{|c|c|c|c|c|c|c|}
\hline \multirow{2}{*}{$\mathrm{N}$} & \multicolumn{4}{|c|}{ Mole Fraction Percent } & \multirow{2}{*}{$\begin{array}{c}\text { Lag } \\
\text { Time } \\
\text { (hours) }\end{array}$} & \multirow{2}{*}{$\begin{array}{c}\mathrm{J} \\
\left(\mathrm{ug} / \mathrm{h} \cdot \mathrm{cm}^{2}\right)\end{array}$} \\
\hline & PG & $\mathrm{OA}$ & ET & DM & & \\
\hline 1 & 1 & 10 & 10 & 79 & $4.0 \pm 0.7$ & $10.7 \pm 4$ \\
\hline 2 & 10 & 10 & 10 & 70 & $4.2 \pm 0.8$ & $11.0 \pm 1$ \\
\hline 3 & 20 & 10 & 10 & 60 & $12.3 \pm 2.2$ & $3.5 \pm 2$ \\
\hline 4 & 30 & 10 & 10 & 50 & $10.0 \pm 1.7$ & $4.2 \pm 1$ \\
\hline 5 & 40 & 10 & 10 & 40 & $12.0 \pm 2.6$ & $16.0 \pm 6$ \\
\hline 6 & 50 & 10 & 10 & 30 & $10.1 \pm 4.3$ & $127.8 \pm 134$ \\
\hline 7 & 60 & 10 & 10 & 20 & $5.1 \pm 0.6$ & $216.2 \pm 44$ \\
\hline 8 & 70 & 10 & 10 & 10 & $2.4 \pm 1.0$ & $148.9 \pm 61$ \\
\hline 9 & 79 & 10 & 10 & 1 & $0.7 \pm 0.4$ & $101.0 \pm 12$ \\
\hline
\end{tabular}

KEY: $\mathbf{N}=$ formulation designation; $\mathbf{J}==$ flux in $\mu \mathrm{g} / \mathrm{h} \bullet \mathrm{cm}^{2}$.

Formulations containing less than $30-40 \%$ DM displayed $Q$ versus $\mathrm{T}$ curves 


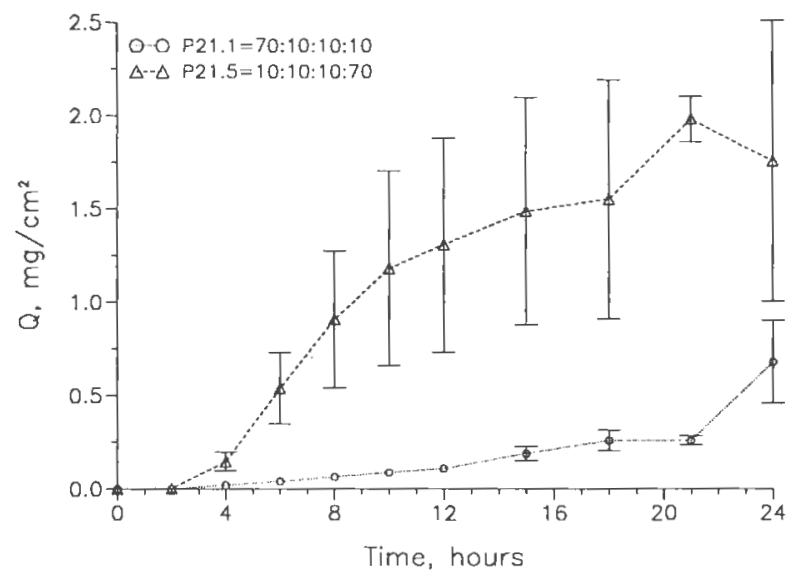

Figure 17. Effect of high and low levels of dimethylisosorbide in quaternary mixtures holding ratio of OA:ET at $10: 10, n=4$.

that leveled out at around 18 hours. While each factor-level combination receives the same number of solvent molecules, all donor formulations consist of suspensions of $10 \%$ excess nifedipine so that the amount of nifedipine actually applied to the skin does vary as a function of nifedipine solubility in the formulation. Only rarely did the total amount of nifedipine permeated approach the amount initially applied and these formulation were biphasic rather than asymptotic. Since this asymptotic behavior is restricted to donor formulations at low levels of DM, it was initially believed to result from depletion of DM in the donor. To test this theory, a 
formulation consisting of PG:OA:ET:DM (50:10:10:30) were applied in doses of 6 and $18 \mu \mathrm{mol}$ of cosolvent per cell. In Figure 18, Q versus $\mathrm{T}$ profiles of the lower dose asymptotes; cells receiving the higher dose did not display an asymptote. Upon termination of the experiment, the amount of nifedipine remaining in the donor compartment of those cells showing asymptotes revealed that for the $6 \mu \mathrm{mol}$ dose, the amount of nifedipine permeated is approximately $16 \%$ of the amount initially applied. The amount of nifedipine recovered in the skin was comparatively small $(\approx 3 \%$ of amount permeated). Crystals of nifedipine were visible in all donor compartments and it is assumed that a sufficient excess of drug $(\approx 80 \%)$ remained to maintain the concentration gradient. In terms of nifedipine solubility, donors high in PG and low in DM possess a lower saturation solubility. Also, as DM diffuses into the skin, the donor simultaneously acquires transpired moisture from the skin, the net effect of which is to lower nifedipine solubility and create a diffusional force favoring partition of nifedipine out of the donor and into the skin. Furthermore, it has been proposed that solvents aiter the dermal environment by alteration of hydrogen bonding of dermal water ${ }^{s}$ or by its ability to solubilize drug. This combination of events alters drug solubility during the experimental period in a manner that favors nifedipine permeation and is consistent with the observation of asymptotic or biphasic behavior. The expression of formulation constituents as mole fraction permits a unique opportunity to model the diffusion process from a stoichiometric perspective. Adoption of this reference system permits the experimental data to be modeled in a meaningful way that illuminates the relative importance of the individual components 


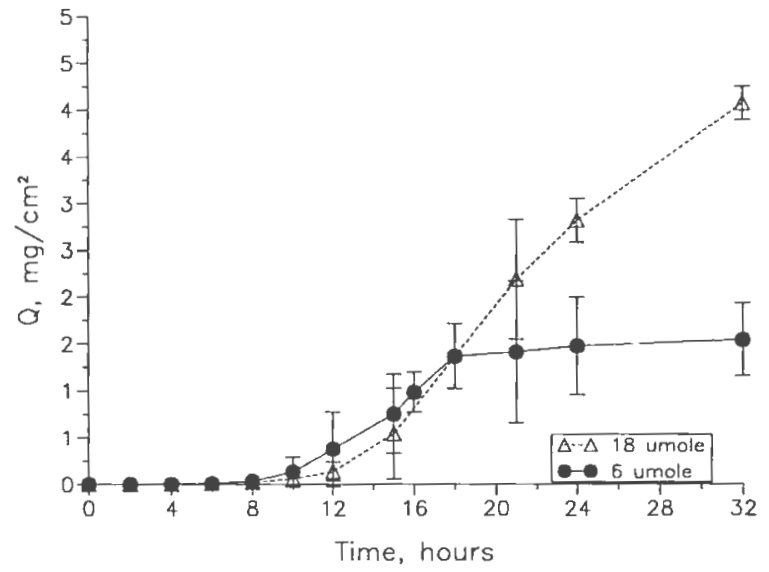

Figure 18. Effect of trebling amount of cosolvent applied from 6 to 18 micromoles. and, more importantly, better demonstrate the potential interaction between components. Regression techniques applied to the data in Table XI resulted in a model predictive of flux and lag time. The criterion for best fit were consideration of the correlation coefficient, residual pattern, sum of squared residuals among the various models tested. The parameter estimates are shown in Table XIII and the significance of the regression is shown in Table XIV. The magnitude of the parameter estimates in Table XIII reveal that all four main effects were not significant. Of the six possible two-way interactions, the synergistic blending of OA appears to be real since all $\beta_{\mathrm{ij}}$ for $\mathrm{i}=\mathrm{j}=2$ terms are greater than zero. Synergism 
among the other components may not be real, since $\beta_{\mathrm{ij}}$ is not significantly greater than zero based on the comparison of each coefficient estimate to the value of its standard error in the form of an approximate Student's t-statistic and then comparing each computed value of $t$ to the tabled value $t_{15,0.25}$. The high standard ertor terms. due in large measure to biological variability of the skin, did not detract from the fitting of the polynomial and the error due to regression $\left(r^{2}=0.83\right)$ is acceptable. The sign of the $\beta$ 's and the corresponding value of the t-statistic lead us to infer that most individual terms are antagonistic toward one another in overall formulation performance while combinations of $\mathrm{X}_{2}$ (i.e., oleic acid) strongly interacts with the other three factors. This result was anticipated, since the components had poor permeation profiles when applied individually. Furthermore, the magnitude of the interaction terms are large relative to the single factor terms and probably represents the driving force for enhanced permeability in these systems.

The elucidation of the synergistic interactions among vehicle components responsible for the superior performance of these quatemary systems is a conclusion difficult to identify in four factor space and emphasizes the importance of treating the data to this type of analysis. Gross observation of the $Q$ versus t profiles would have led to the (incorrect) conclusion that DMI had a deleterious effect on permeation. However, the model predicts that it is not so much the main effects, but rather the interaction between $\mathrm{OA}$ and the other three components which explains enhanced permeation.

Since all four factors affect the response, it is not possible to identify a region 
Table XIII. Parameter estimates from data presented in Table XI.

\begin{tabular}{|c|c|c|c|c|c|}
\hline \multicolumn{6}{|c|}{ Parameter Estimates } \\
\hline Variable & $\mathrm{DF}$ & $\begin{array}{c}\text { Parameter } \\
\text { Estimate }\end{array}$ & $\begin{array}{l}\text { Standard } \\
\text { Error }\end{array}$ & $\mathrm{t}^{2}$ & $P>|T|$ \\
\hline$X_{1}$ & 1 & 100.5 & 27.1 & 3.715 & 0.0016 \\
\hline $\mathrm{X}_{2}$ & 1 & -3369.0 & 1588.1 & -2.121 & 0.0480 \\
\hline$X_{3}$ & 1 & -1773.4 & 1605.6 & -1.105 & 0.2839 \\
\hline$X_{4}$ & 1 & -146.3 & 111.1 & -1.317 & 0.2044 \\
\hline $\mathrm{X}_{1} * \mathrm{X}_{2}$ & 1 & 3990.3 & 1912.4 & 2.087 & 0.0514 \\
\hline $\mathrm{X}_{1} * \mathrm{X}_{3}$ & 1 & 2098.5 & 1898.5 & 1.105 & 0.2836 \\
\hline$X_{1} * X_{4}$ & 1 & -14.7 & 167.3 & -0.088 & 0.9308 \\
\hline $\mathrm{X}_{2} * \mathrm{X}_{3}$ & 1 & 9573.7 & 4502.8 & 2.126 & 0.0476 \\
\hline$X_{2} * X_{4}$ & 1 & 4658.8 & 2257.0 & 2.064 & 0.0537 \\
\hline$X_{3} * X_{4}$ & 1 & 1945.4 & 2356.0 & 0.826 & 0.4198 \\
\hline
\end{tabular}

${ }^{1} \mathrm{X}_{1}=$ propylene glycol; $\mathrm{X}_{2}=$ oleic acid; $\mathrm{X}_{3}=$ ethanol; $\mathrm{X}_{4}=$ dimethylisosorbide.

${ }^{2} t=$ computed value of Student's t-statistic (tabled value $t_{15.0 .25}=0.691$ ).

of maximum (or minimum) response in three-dimensional terms because they are hyperplanes in four-dimensional space. Futhermore, the canonical analysis of the fitted surface yield negative and positive eigenvalues, indicative of a saddle region of "good" formulations rather than a unique optimum point for both flux and lag time. Therefore, although a predictive model has been developed, it is unlikely that for this multi-response problem there exists a unique combination of factors which result in optimum values for both responses simultaneously. 
Table XIV. Analysis of Variance of data presented in Table XI.

\begin{tabular}{||c|c|c|c|c|c||}
\hline \multicolumn{7}{|c||}{ Analysis of Variance } \\
\hline \multirow{2}{*}{ Source } & DF & $\begin{array}{c}\text { Sum of } \\
\text { Squares }\end{array}$ & $\begin{array}{c}\text { Mean } \\
\text { Square }\end{array}$ & F Value & Prob > F \\
\hline Model & 10 & 212635.7 & 212653.6 & 14.4 & 0.0001 \\
\hline Error & 18 & 26579.1 & 1476.6 & \\
\hline Total & 28 & 239214.8 & & \\
\hline & Root MSE $=38.4$ & & \\
\cline { 2 - 6 } & Dep Mean $=75.3$ & \multicolumn{3}{c||}{ R-square $=0.8889$} \\
\cline { 2 - 6 } & \multicolumn{6}{|c|}{ C. V. $=51.0$} & \multicolumn{3}{c|}{ Adj R-sq $=0.8272$} \\
\hline
\end{tabular}

The predicted response surface for flux (Figure 19) and lag time (Figure 20) correspond exclusively to systems in which the level of both oleic acid and ethanol is held at the mole fraction level of $10 \%$. Unfortunately, since all four two-way interactions with OA and the remaining factors affect the response, such plots cannot be readily used to identify a region of maximum (or minimum) response for the present $n+4$ factor space. One solution is to set constraints on lag time and optimize flux subject to this constraint. As an alternative, a ridge analysis is used to locate the range of 'optimum' response within the design space. As a second alternative, the predicted response surface for flux within the constraints of three factors may be examined at discrete levels of the fourth factor in a manner analogous to a radiological CAT scan. Such conceptualizations, produced under the SAS ADX system, offer an advantage over the three-dimensional plot in Figure 19 in that they 
Table XV. Ridge analysis of data in Table XI.

\begin{tabular}{|c|c|c|c|c|c|}
\hline \multicolumn{6}{|c|}{--- Type of ridge = MINIMUM ---- } \\
\hline $\mathrm{X}_{1}$ & $\mathrm{X}_{2}$ & $\mathrm{X}_{3}$ & $X_{4}$ & PRED & STERR \\
\hline 0.49 & 0.11 & 0.11 & 0.29 & 89.07 & 16.82 \\
\hline 0.45 & 0.10 & 0.10 & 0.34 & 77.73 & 17.44 \\
\hline 0.41 & 0.10 & 0.10 & 0.39 & 66.10 & 17.99 \\
\hline 0.37 & 0.09 & 0.10 & 0.44 & 54.09 & 18.66 \\
\hline 0.35 & 0.08 & 0.09 & 0.48 & 41.58 & 19.78 \\
\hline 0.32 & 0.07 & 0.09 & 0.52 & 28.45 & 21.86 \\
\hline 0.29 & 0.06 & 0.09 & 0.56 & 14.56 & 25.31 \\
\hline 0.26 & 0.05 & 0.09 & 0.60 & 0.20 & 30.31 \\
\hline 0.24 & 0.04 & 0.10 & 0.63 & 0.0 & 36.83 \\
\hline 0.22 & 0.03 & 0.10 & 0.66 & 0.0 & 44.69 \\
\hline 0.20 & 0.01 & 0.10 & 0.69 & 0.0 & 53.70 \\
\hline \multicolumn{6}{|c|}{ Type of ridge = MAXIMUM } \\
\hline 0.49 & 0.11 & 0.11 & 0.29 & 89.07 & 16.82 \\
\hline 0.53 & 0.13 & 0.11 & 0.24 & 100.20 & 16.22 \\
\hline 0.58 & 0.12 & 0.12 & 0.19 & 111.17 & 15.81 \\
\hline 0.62 & 0.12 & 0.12 & 0.14 & 122.05 & 16.16 \\
\hline 0.66 & 0.12 & 0.12 & 0.09 & 132.88 & 17.80 \\
\hline 0.71 & 0.12 & 0.13 & 0.04 & 143.56 & 21.03 \\
\hline
\end{tabular}

$\mathrm{X}_{1}=$ propylene glycol; $\mathrm{X}_{2}=$ oleic acid; $\mathrm{X}_{3}=$ ethanol; $\mathrm{X}_{4}=$ dimethylisosorbide

permit visualization of three rather than two factors (Figure 21 through Figure 24). 


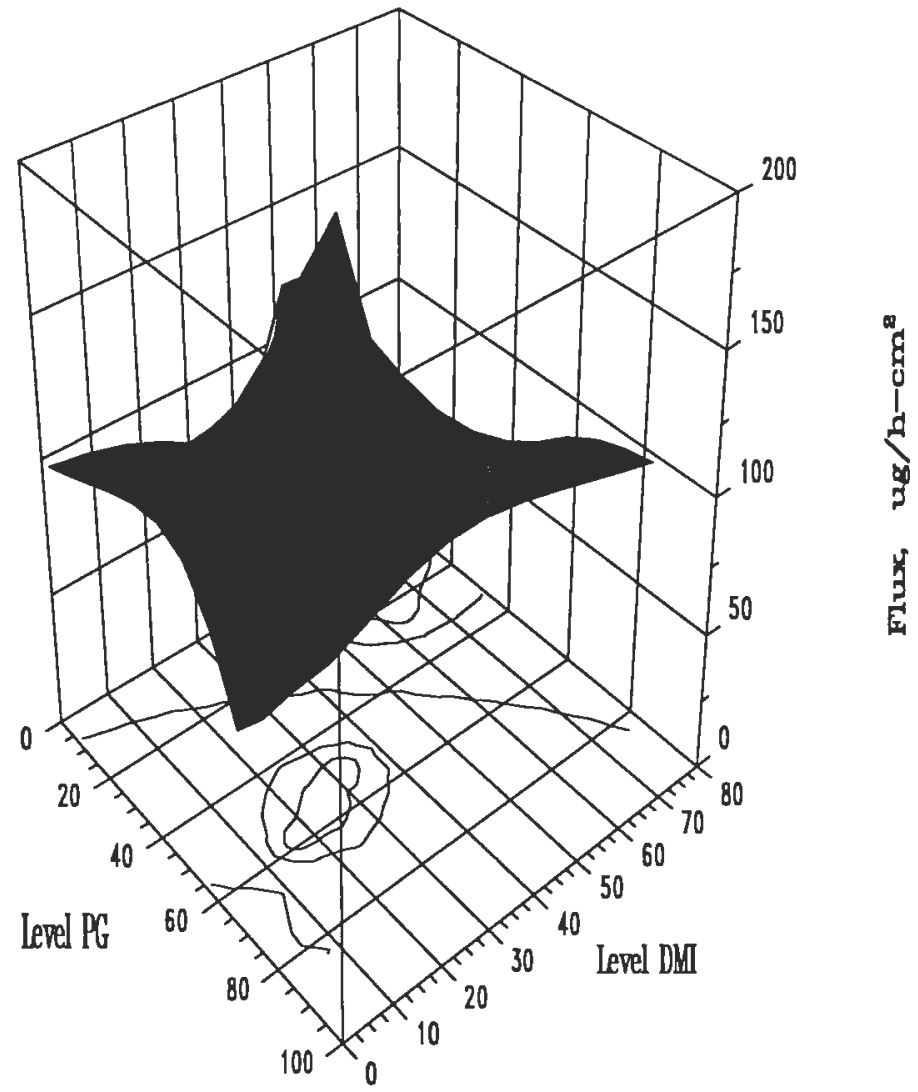

Figure 19. Three dimensional response surface and contour map of the predicted flux in quatemary mixtures studied (oleic acid and ethanol both at the $10 \%$ level). 


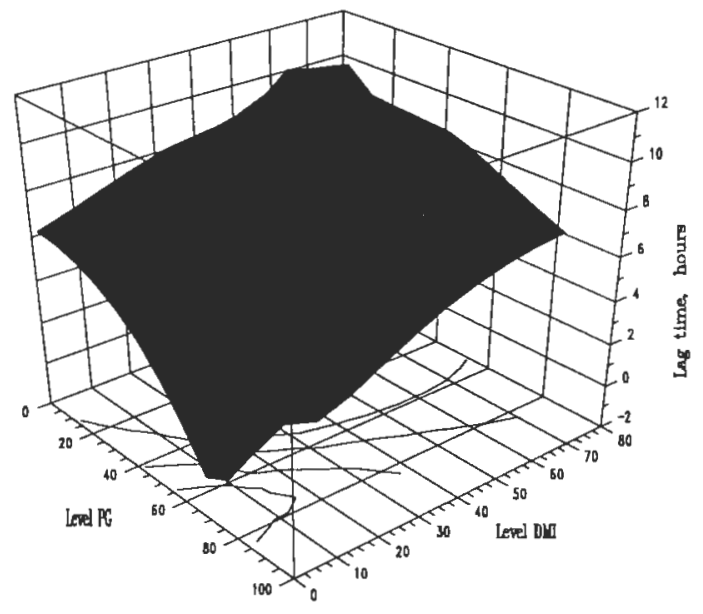

Figure 20. Three dimensional response surface and contour map of the predicted lag time in the quaternary mixtures studied (oleic acid and ethanol both at the $10 \%$ level). 


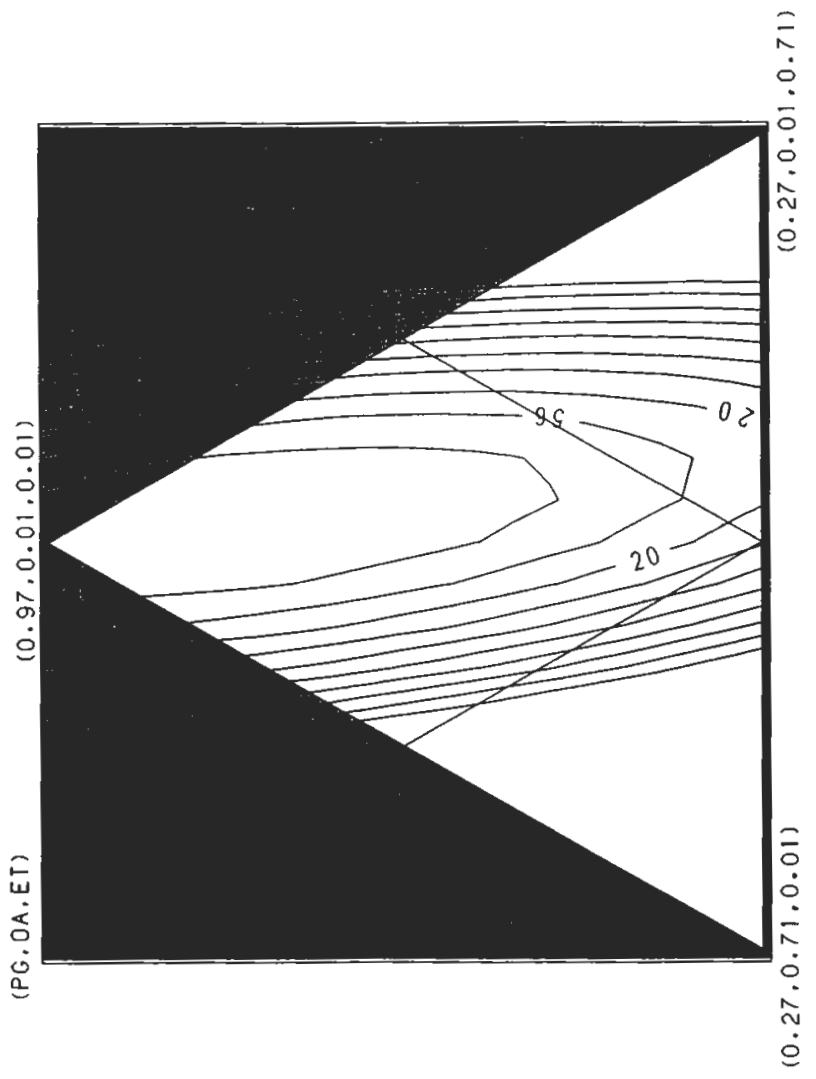

Figure 21. Contours of the predicted flux for polyhedron in Figure 16: DMI held at $1 \%$. 


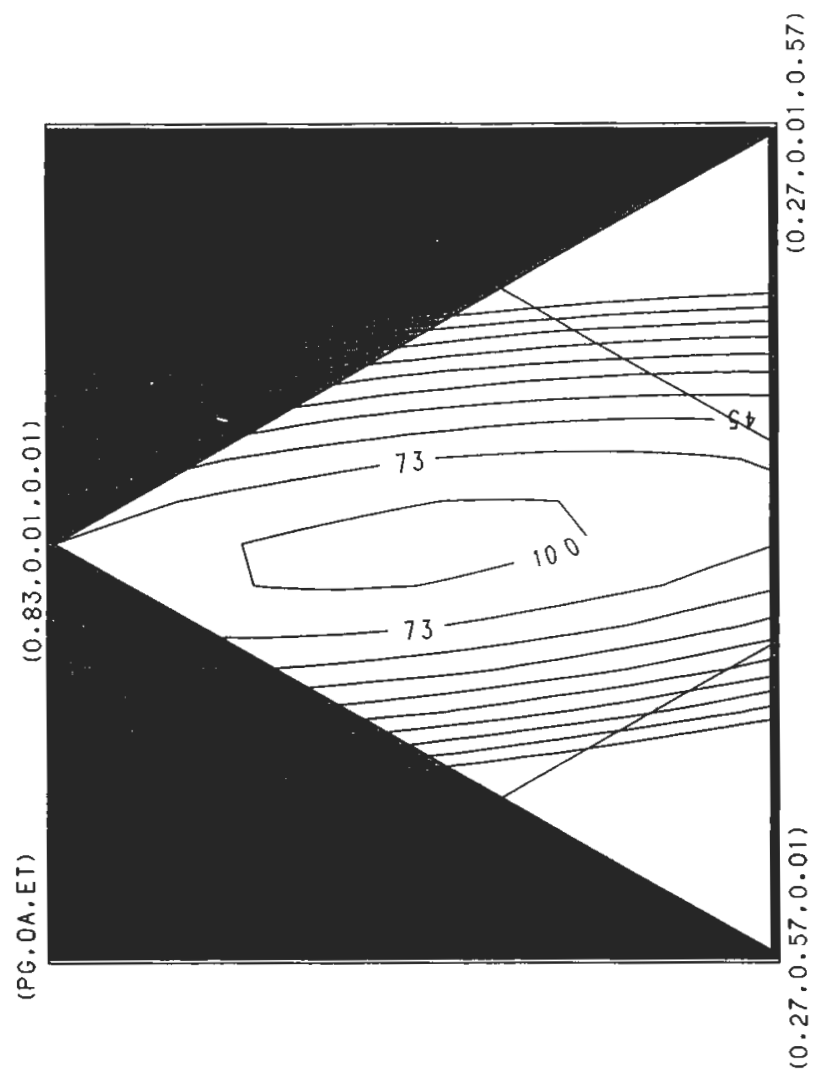

Figure 22. Contours of the predicted flux for polyhedron in Figure 16: DMI held at $15 \%$. 


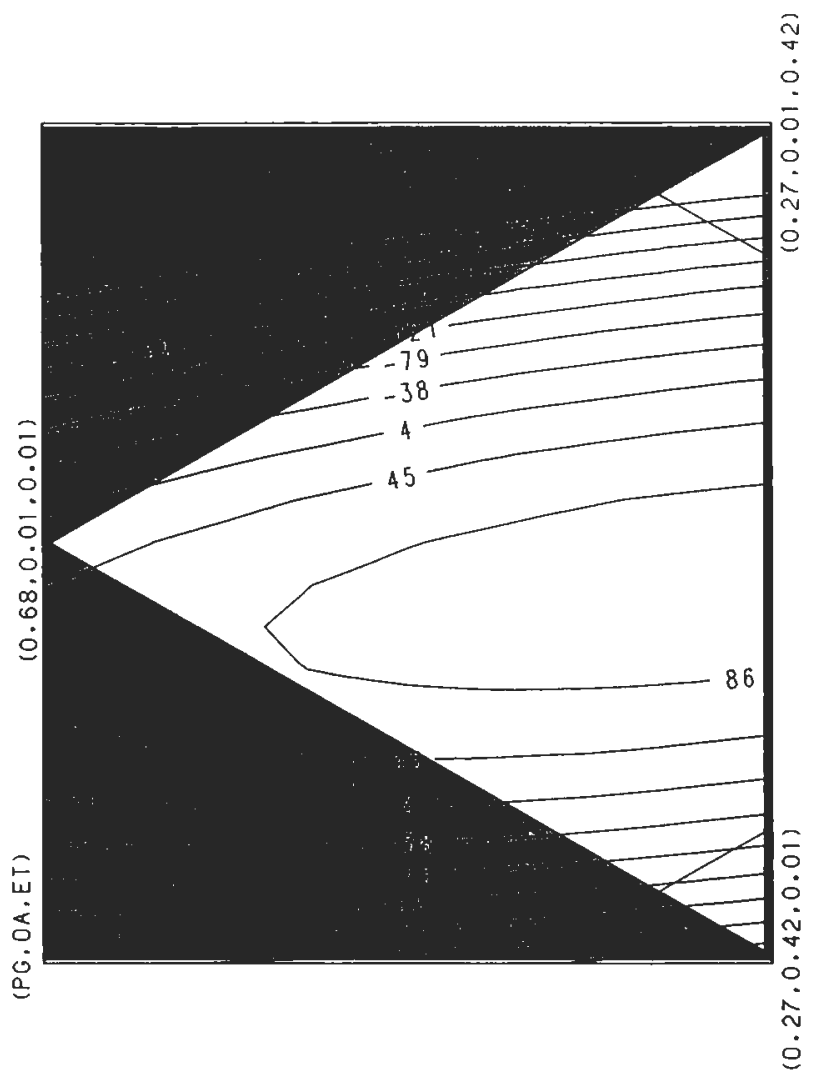

Figure 23. Contours of the predicted flux for polyhedron in Figure 16: DMI held at $30 \%$. 


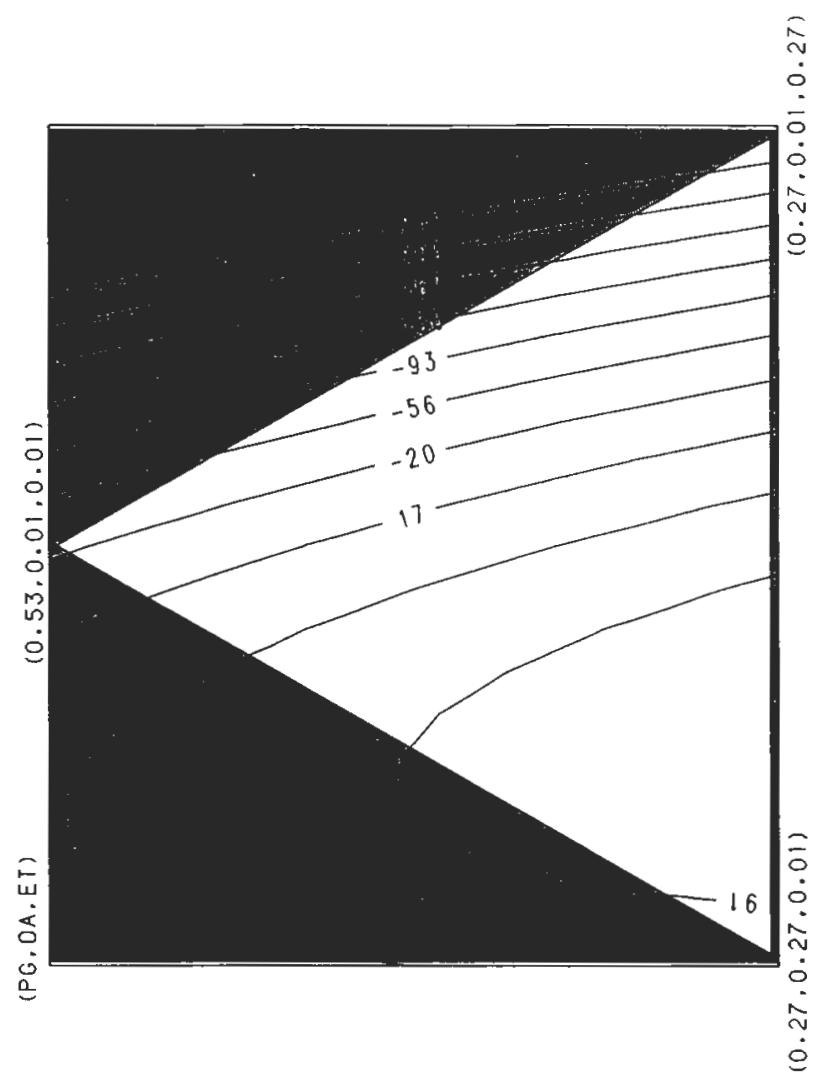

Figure 24. Contours of the predicted flux for polyhedron in Figure 16: DMI held at $45 \%$. 


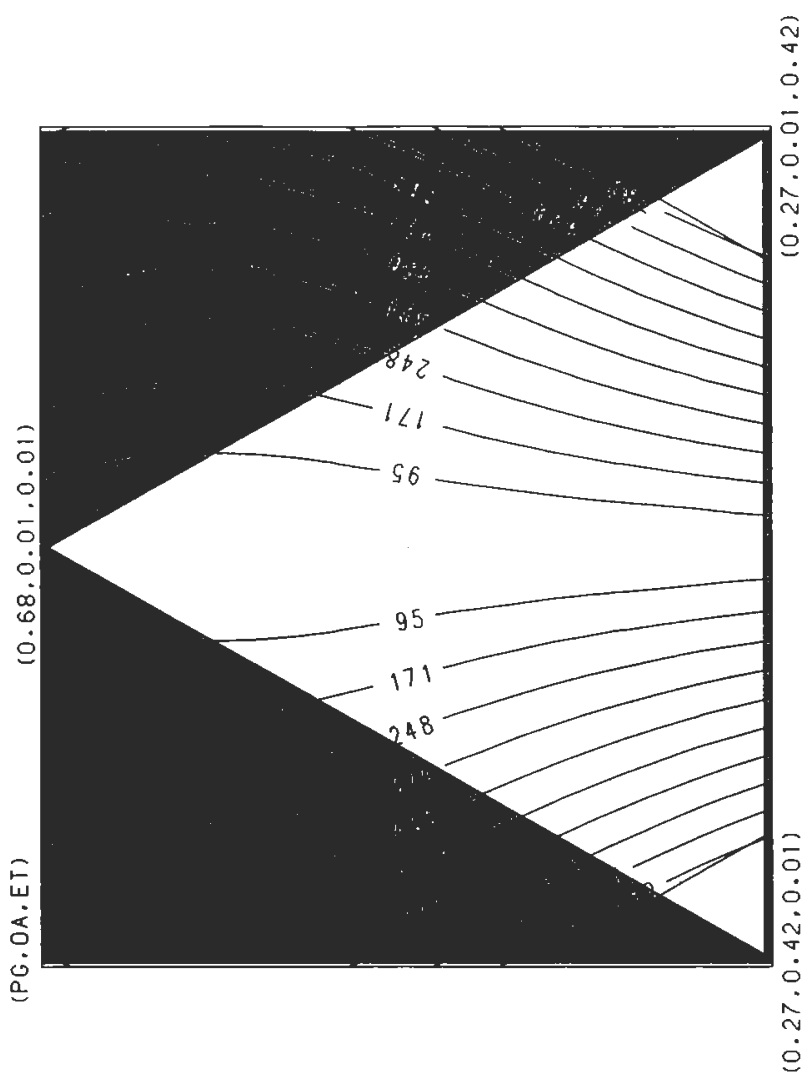

Figure 25. Contours of the standard error of predicted flux responses for formulations in Figure 23. 


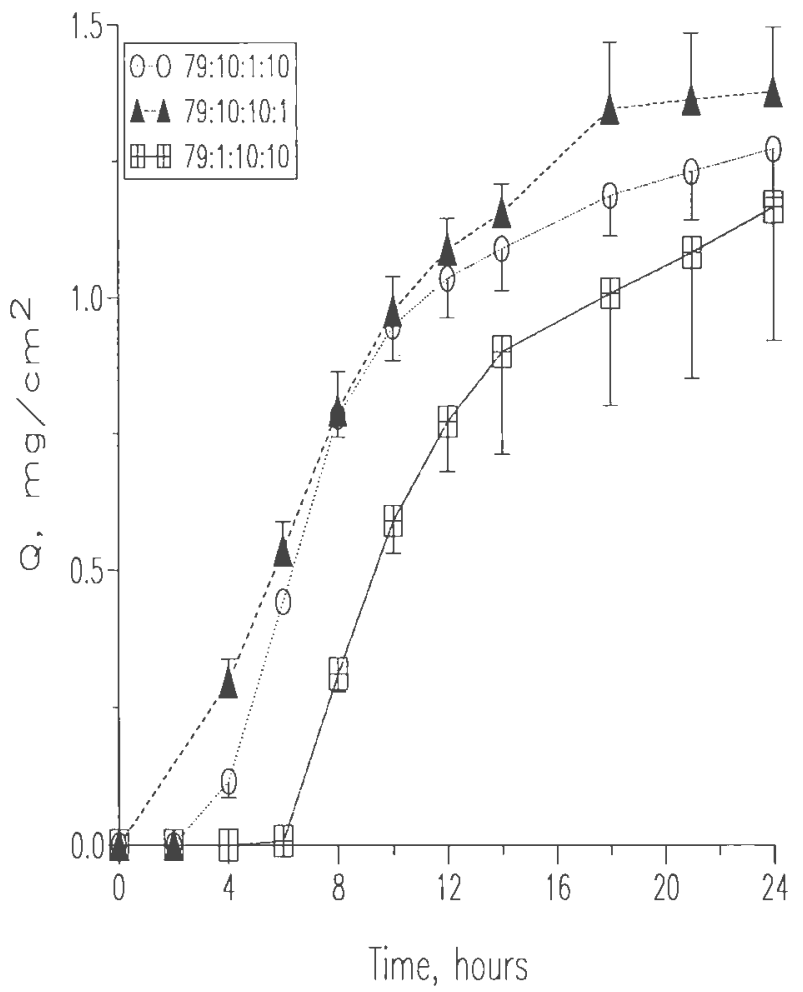

Figure 26. Permeation of nifedipine from formulations of high propylene glycol content. 
The eigenvalues and eigenvectors obtained from regression analysis predict elliptical response surfaces for both lag time and flux that are most sensitive to changes in the levels of $\mathrm{OA}$ and DM, while the level of PG relative to these key components should not influence the slope of either response surface as significantly. This behavior is readily apparent in Figure 21 through Figure 24 which are serial slices taken along the DMI (i.e., $\mathrm{X}_{4}$ ) axis of the theoretical sample space represented by the polyhedron in Figure 16. Data treated in this manner permits visualization of the contour map of fluxes predicted from mixtures of PG, OA and ET at discrete levels of DMI, Determination of the composition of any formulation within the confines of the triangular space conforms with the rules governing standard ternary phase diagrams. The contour intervals running parallel to the PG axis (vertically) indicate that the flux response is least sensitive to this component. Regions having narrow contour intervals, such as those parallel to the base of Figure 21, predict that the flux response will change rapidly with changes corresponding to horizontal movement from regions of low to high oleic acid. Clearly, movement in this direction results in rapid achievement of optimal fluxes followed by equally rapid decline in predicted permeation rates. An additional feature of the system is that it also provides a measure of confidence in the response estimates via plotting of the standard error as shown in Figure 25 . These results suggest that a relatively high level of PG and low levels of OA, ET and DM will give maximum flux and minimum lag times.

From a stoichiometric perspective, the diffusion process to be envisioned as 
one in which OA and DM are the rate limiting factors, while ET and PG have less activity and are present in excess. Based on these results of the regression, optimal performance should be realized from a formulation high in $\mathrm{PG}$ relative to the other components. A compromise is accepted in formulation 9 in Table XII (i.e., PG:OA:ET:DM = 79:10:10:1) which optimize flux to $100.99 \pm 2 \mu \mathrm{g} / \mathrm{h} \cdot \mathrm{cm}^{2}$ while holding the lag time under six hours. The effect of OA, ET and DM concentrations relative to each other while holding the level of PG at $79 \%$ are shown in Figure 26. The results of these permeation experiments conform to predictions based on eigenvalues obtained from the model obtained from the data in Table X

The minimum and maximum predicted flux response values expected from various formulation combinations are shown in Table XV. The maximum flux predicted by the model was expected from a mole fraction ratio of $70: 12: 13: 5$, the permeation results which are presented in Figure 15. Note that this ridge maximum is outside the constraints of the polyhedron within Figure 16. and the amount of error likely to be incurred generally rises on the periphery of the experimental space. Figure 25 shows that all approximation are expected to be particularly imprecise near the edges of the experimental region determined by the design space. This optimized formulation (designated P42.1) yielded a flux of $60 \pm 6 \mu \mathrm{g} / \mathrm{h} \cdot \mathrm{cm}^{2}$ and a lag time of only 2 hours which is twice that required to deliver an equivalent oral daily dose.

In summary, regression analysis have been successfully applied to data obtained from permeation experiments to identify positive synergistic interactions among formulation components. The intractable nature of mixture designs was 
overcome by using a Mclean Anderson experimental design which permitted the results of the permeation studies to be interpreted in a stoichiometric fashion. Mathematical techniques successfully modeled the permeation process so that quantitative predictions of the flux or lag time could be made in $n+3$ dimensional space. These observations support conclusions reached earlier regarding the importance of solubility of the permeant in the donor phase, the codiffusion of solvent with drug, and the general conditions necessary to promote the percutaneous absorption of compounds with poor solubility profiles. This novel approach facilitated the rational selection of components in transdermal dosage forms that permitted systemic drug availability of a magnitude required for potential therapeutic response. 


\section{REFERENCES}

1. Comell, J.A. EXPERIMENTS WITH MIXTURES: Designs, Models, and the Analysis of Mixture Data New York: John Wiley \& Sons 1983.

2. Submitted for publication.

3. McLean, R.A.; Anderson V.L. "Extreme Vertices Design of Mixture Experiments." Technometrics. 1966, 8, 447-454.

4. Imperial Chemical Industries product literature.

5. Scheuplein,R.; Ross, L. "Effects of Surfactants and Solvents on the Permeability of Epidermis." J.Soc.Cosm.Chem. 21 (December 1970): 853-74. 
APPENDICES 


\author{
APPENDIX A: \\ COMPUTER PROGRAM SOURCE CODE \\ DESCRIPTION AND LISTINGS
}

\title{
INTRODUCTION
}

This appendix describes the computer programs written for this study. Since screening studies typically generate large quantities of data, standard operating procedures for data coding, storage and processing that are both rugged and robust are of inestimable value in all areas of research. In addition, optimization problems, which are numerically intensive, have evolved and now permeate many areas of research. For these reasons, an IBM mainframe was employed, for it offered virtually unlimited access to storage and data processing capabilities. The language chosen for this work was the SAS language running under the FSVM environment on the Academic Computer Center IBM mainframe.

This section provides an overview of the general purpose of the two programs entitled CELLS3 SAS A1 and SIMPLEX SAS A1. Briefly, the function of CELLS3 SAS A 1 is calculate flux and lag times by determining the amount of nifedipine permeated versus time. The source code is provided because it utilizes an algorithm for handling large, complex data sets that can be applied to many fields of research. 
The source code for SIMPLEX SAS Al is also supplied as it shows the means by which the various properties of the formulation studied were calculated.

In our laboratory, a Waters Baseline 810 chromatography workstation has been programmed to acquire data under its Summary option to allow storage of data on floppy disk to be uploaded to the mainframe via a modem telecommunications link. A novel method for coding the individual data points was developed. This scheme was successfully utilized by six researchers sharing the apparatus to manage thousands of data points without mishap. In addition, it provides a functionally useful format to be read by subsequent data processing steps in the SAS system. The specifics of this program are discussed in detail in the section titled "CELLS3 SAS A1".

\section{CELLS3 SAS A1}

A particularly useful format for maintaining integrity of the data sets that also provides for efficient identification and retrieval bas been to code the data points in the following hierarchal structure: assay, notebook page, formulation reference, replicate number (i.e., variables V1 thorough V4) followed by the drive unit position, sample interval, and the time the sample was taken. This is accomplished by first allocating space in a hard disk subdirectory and then assigning each chromatogram a unique 8 digit filename and sample name in the "LOAD QUE" option. Following data acquisition and storage as an ASCII file on floppy disk, the sample names (which are comprised of the four identifiers V1 thorough V4 assigned in the "LOAD QUE" 
step) are quickly expanded, using the "SEARCH/REPLACE" feature of any wordprocessor, to the format utilized by the INPUT statement in the data step DATA ASCON in program CELLS3 SAS A1. CELLS3 SAS Al was written in modular fashion using the SAS macro facilities which allow for a great deal of versatility. Results from the analysis can be stored in a sequential text file with the following format: (1) method (2) notebook (3) formulation, (4) replicate, (5) etc. "Method" refers to the Baseline 810 program containing the instructions used to inject, acquire, and process chromatograms and calculate the amount of nifedipine using peak areas by the external standard method.

It is essential that the user of the SAS ADX ${ }^{\mathrm{TM}}$ menu (SAS Institute Inc. Carey, NC) be aware that the device driver of the present version of SAS (6.01) at this installation must be modified in order to obtain hardcopy of graphics produced. Prior to invoking the SAS ADX system at URI, the user must insure that the following essential steps have been properly executed:

(1) the Q2200PS driver has been modified from REPLACE to APPEND.

(2) the following statements :

X FILE GSASFILE DISK filename filetype filemode; GOPTIONS SWAP GACCESS=GSASFILE DEVICE=Q2200PS; RUN; are submitted by the user from the Display Manager Screen prior to invoking the SAS ADX system. The first statement instructs the mainframe where to hold the graphics, while the second statement configures the SAS system to URI standards. The SAS ADX system is invoked from the Display Manager by typing DESIGN on the 
program line. At this point, the user may engage in an interactive session with the SAS ADX menu system that conforms to those instructions found in the manual. ${ }^{1}$

ADX is configured to display color graphics upon any graphics terminal by specifying "DEVICE $=$ TEK 4107 " when prompted by the system. Direction of graphics output from the SAS ADX system to the University laser printer requires that the user exit the ADX system and at the CMS READY prompt type FILEL, tab down to the 'filename filetype filemode' (i.e., the repository of the graphic stream provided to the system in the statement above) and typing the following command RPRINT / LPIPS

upon which the graphic image may be recovered at the Dispatch Office in Tyler Hall.

${ }^{1}$ Brown, Jason J. and Randall D. Tobias, ADX Menu System Examples: Preliminary Version, February 1991. Statistical Quality Improvement R \& D Applications Division, SAS Institute Inc. SAS Campus Drive Carey, NC 27513. 


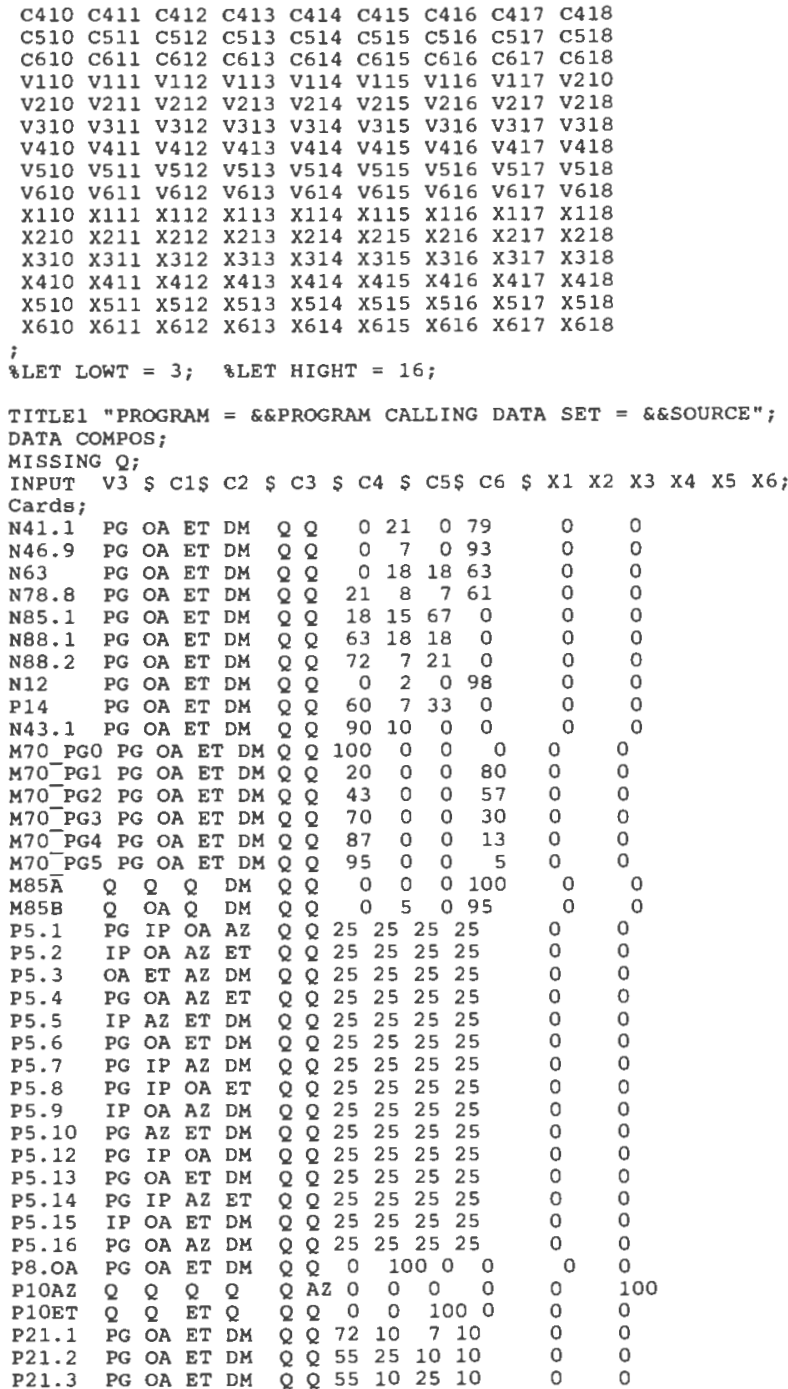




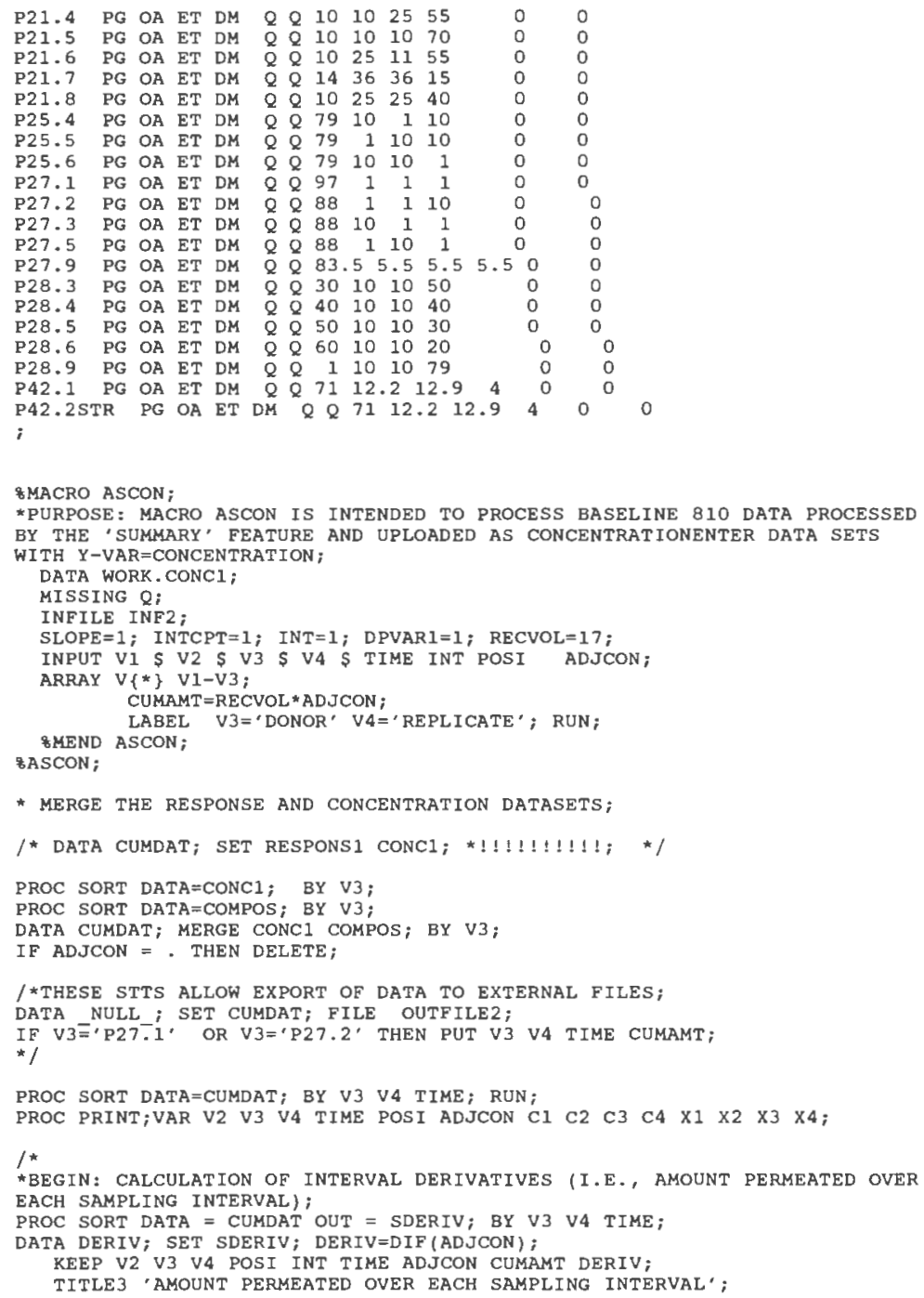


TITLE4 'DERIVATIVES SORTED BY V3 V4 POSI AND TIME';

PROC PRINT NOOBS; RUN;

*END: CALCULATE INTERVAL DERIVATIVES;

*/

PROC SORT DATA=CUMDAT OUT=CUMDAT; BY V3 V4 TIME;

DATA WORK. SEP;

DATA SEP $\Rightarrow$ FUNCTION IS TO PERFORM THE FOLLOWING:

1. CREATE A VARIABLE 'EOFF' TO CONTAIN AN END OF FILE INDICATOR,

2. RETAIN THE VALUE OF ' $J$ ' FROM PREVIOUS INTERATION OF DATA STEP,

3. AND USE THE SYMPUT FUNCTION TO CREATE A MACROVARIABLE, 'JM',

AND ASSIGN TO IT A VALUE FROM THE CURRENT DATA SET'S ITERATION (IE. ' $J$ ') NOTING THAT THE INITIAL VALUE OF $J$ WAS 0 ;

SET WORK, CUMDAT END=EOFF;

BY V3-V4;

RETAIN J;IF FIRST.V3 THEN $J+1$;

IF EOFF THEN CALL SYMPUT('JM',J);

IF EOFF THEN CALL SYMPUT'('NUM',J);

IF EOFF THEN CALL SYMPUT('TOTAL',_N_);

RUN;

MACRO CREATE1;/*SEPARATE INTO DISCRETE DATASETS BY FORMULATION*/

OLOCAL $\mathrm{H}$;

\&LET $\mathrm{H}=1$;

\&DO \&WHILE (\&H<=EJM);

DATA WORK. EXDATAEH;

SET WORK.SEP;

IF $\mathrm{J}=\mathrm{QH}_{\mathrm{H}}$

* IF TIME > 26 THEN DELETE;

CALL SYMPUT('XI'| $\operatorname{LEFT}(\mathrm{J}), \operatorname{TRIM}(\mathrm{X} 1))$;

CALL SYMPUT('X2'| $(\operatorname{LEFT}(\mathrm{J}), \operatorname{TRIM}(\mathrm{X} 2))$;

CALL SYMPUT' $\left(X^{\prime}, \mid L E F T(J), T R I M(X 3)\right)$;

CALL SYMPUT ('X4' $\mid$ LEFT(J), TRIM(X4));

CALL SYMPUT('Cl' LEFT(J), TRIM(C1));

CALL SYMPUT('C2'; LEFT(J),TRIM(C2));

CALL SYMPUT ('C3' LEFT(J), TRIM(C3));

CALL SYMPUT('C4 ' | LEFT(J),TRIM(C4));

CALL SYMPUT('V1' LEFT(J), TRIM(V1));

CALL SYMPUT('V2' (LEFT(J), TRIM(V2));

CALL SYMPUT' 'V3' LEFT(J), TRIM(V3)):

CALL SYMPUT('V4'||LEFT(J),TRIM(V4));

PROC PRINT DATA=WORK. EXDATA\&H NOOBS;

TITLE2

"OUTPUT: DATASET WORKEXDATA \&H (MACROPRINT)" ;

TITLE 3

"FORMULATION \&\&V3\&H ASSAYED BY\&\&VI\&H (SEE NOTEBOOK PAGE=\&\&V2\&H)"; TITLE4

"DONOR: $\& \& C 1 \& H=\& \& X 1 \& H \quad \& \& C 2 \& H=\& \& X 2 \& H \quad \& \& C 3 \& H=\& \& X 3 \& H \quad \& \& C 4 \& H=\& \& X 4 \& H "$; TITLE5

" $\quad \& \& C 5 \& \mathrm{H}=\& \& \times 5 \& \mathrm{H} \quad \& \& C 6 \& \mathrm{H}=\& \& \times 6 \& \mathrm{H} "$;

BY $\mathrm{V} 3-\mathrm{V} 4$;

VAR VI V2 V3 V4 INT TIME POSI DPVAR1 ADJCON CUMAMT;

LABEL V2='NB PAGE:' V3='DONOR:' V4='REPLICATE:'; RUN;

PROC SORT DATA=EXDATA\&H OUT=WORK. EXDATA\&H; BY V3 TIME DPVAR1;

PROC MEANS DATA EXXDATA\&H

MAXDEC $=2$ N MEAN STD STDERR MIN MAX CV 


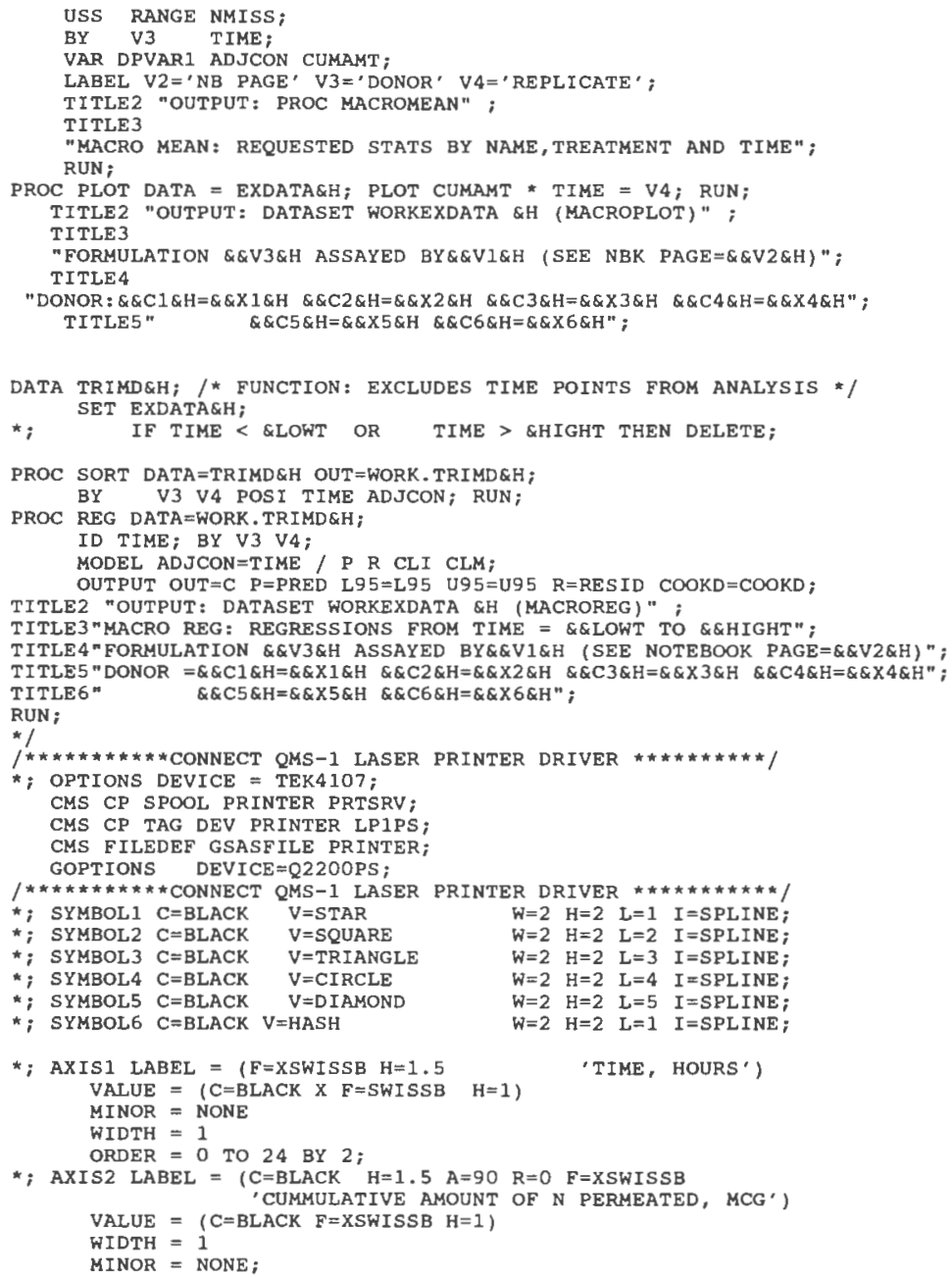

FOOTNOTE1"SOURCE: DATASET: \&\&SOURCE BY PROGRAM: \&\&PROGRAM"; FOOTNOTE2 $\mathrm{J}=\mathrm{L} \quad \mathrm{H}=1 \quad \mathrm{~F}=\mathrm{COMPLEX} \quad \mathrm{C}=\mathrm{BLACK}$ 
'MEMBRANE SURFACE AREA = $4.9 \mathrm{SQCM}$ ';

TITLE2 C=BLACK $\mathrm{H}=1.5 \mathrm{~F}=$ XSWISS

'PERMEATION OF NIFEDIPINE FROM PG:OA:ET:DM SUSPENSIONS';

TITLE 3"MACRO PLOT OUTPUT (TIME = \&LOWT TO \& \&HIGHT) ";

TITLE 4

"FORMULATION \&\&V3\&H ASSAYED BY\&EVIEH (SEE NOTEBOOK PAGE=\&\&V2\&H)" ; TITLE5

"DONOR $=\& \& C 1 \& H=\& \& X 1 \& H \quad \& \& C 2 \& H=\& \& X 2 \& H \quad \& \& C 3 \& H=\& \& X 3 \& H \quad \& \& C 4 \& H=\& \& X 4 \& H "$;

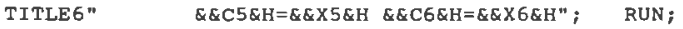

PROC GPLOT DATA = EXDATA\&H;

PLOT CUMAMT TIME $=\mathrm{V} 4$

$/$ VREF $=15000$ HAXIS $=$ AXIS1 VAXIS $=$ AXIS2;

* PLOT ADJCON*TIME='A' PRED*TIME='P' U95*TIME='U' L95*TIME='L'

/ OVERLAY VPOS $=32$ HPOS $=80$;

PLOT RESID TIME / VREF $=0$ VPOS $=18$ HPOS $=60$;

*/

PLOT COOKD*TIME /VREF $=0$ VPOS $=18$ HPOS $=60$;

\&LET H=8EVAL $(\& H+1)$;

QEND;

\&MEND CREATE1;

* NOW CONTROl EXECUTion FROM heRE With macros ;

*; ASCON;

* qASRESPON;

*; SCREATE1;

RUN;

ENDSAS; 


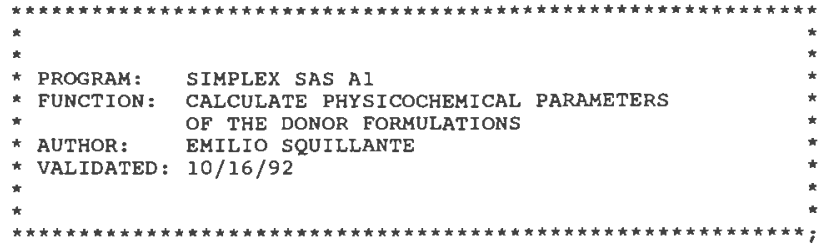

8GLOBAL LOWX2 LOWX3 HIGHX2 HIGHX3 VSIZE HSIZE;

\&LET HSIZE $=0$; \&LET VSIZE $=0$;

\&LET LOWX2 = 9; \&LET HIGHX2 = 11;

8LET LOWX3 = 9; 8LET HIGHX3 = 11;

DATA COMPOS;

MISSING Q;

TITLE1"OUTPUT: SIMPLEX SAS AI (UNBALANCED DATA, NMAX=4)";

INPUT CODE $\$ \mathrm{C} 1 \$ \mathrm{C} 2 \$ \mathrm{C} 3 \$ \mathrm{C} 4 \$ \mathrm{X} 1 \times 2 \times 2 \times 3 \times 4$;

Cards;

N41.1 PG OA ET DM O $021 \quad 079$

N46.9 PG OA ET DM O 7 O 093

$\begin{array}{lllllllll}N 63 & P G & O A & E T & D M & 0 & 18 & 18 & 63\end{array}$

$\begin{array}{llllllll}\text { N78.8 PG OA } & \text { ET DM } & 21 & 8 & 7 & 61\end{array}$

$\begin{array}{lllllllll}\text { N85.1 PG OA } & \text { ET } & \text { DM } & 18 & 15 & 67 & 0\end{array}$

N87.4 PG OA ET DM 1000000

$\begin{array}{lllllllll}\text { N88.1 } & \text { PG } & \text { OA } & \text { ET } & \text { DM } & 63 & 18 & 18 & 0\end{array}$

$\begin{array}{llllllll}N 88.2 & \text { PG OA } & \text { ET } & \text { DM } & 72 & 7 & 21 & 0\end{array}$

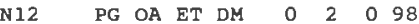

$\begin{array}{llllllll}\text { P14 PG OA ET DM } & 60 & 7 & 33 & 0\end{array}$

$\begin{array}{lllllllllll}\text { P14 PG OA ET } & \text { DH } & 60 & 7 & 33 & 0\end{array}$

N43.1 PG OA ET DM $90 \quad 10 \quad 0 \quad 0$

M70 PGO PG OA ET DM 100 O 00 O

M70-PG1 PG OA ET DM 20 O

M70- PG2 PG OA ET DM 43 O

M70-PG 3 PG OA ET DM 70 0 O 0 30

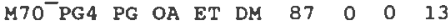

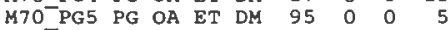

$\begin{array}{lllllllll}M 85 \vec{A} & Q & Q & Q & D M & 0 & 0 & 0 & 100\end{array}$

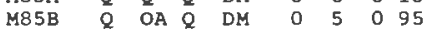

$\begin{array}{llllllll}\text { P5.6 PG OA } & \text { ET } & \text { DH } & 25 & 25 & 25 & 25\end{array}$

P8.OA PG OA ET DH 0 100 000

P1OET PG OA ET DM 0 O 0 100 0

P21.1 PG OA ET DM $72 \quad 10 \quad 7 \quad 10$

$\begin{array}{lllllll}\text { P21.2 PG OA ET DM } & 55 & 25 & 10 & 10\end{array}$

P21.3 PG OA ET DH $5510 \quad 2510$

P21.4 PG OA ET DM $10 \quad 10 \quad 25 \quad 55$

P21.5 PG OA ET DH $10 \begin{array}{llll}10 & 10 & 70\end{array}$

P21.6 PG OA ET DM $10 \quad 25 \quad 11 \quad 55$

P21.7 PG OA ET DM $14 \begin{array}{lllll}36 & 36 & 15\end{array}$

P21.8 PG OA ET DM $10 \quad 25 \quad 25 \quad 40$

P25.4 PG OA ET DM $79 \begin{array}{llll}10 & 1 & 10\end{array}$

P25.5 PG OA ET DH $79 \quad 1 \quad 10 \quad 10$

$\begin{array}{llllllll}\text { P25.6 PG OA } & \text { ET } & \text { DM } & 79 & 10 & 10 & 1\end{array}$

$\begin{array}{lllllll}\text { P27.1 PG OA } & \text { ET DM } & 97 & 1 & 1 & 1\end{array}$

$\begin{array}{lllllllll}\text { P27.2 } & \text { PG } & O A & E T & D M & 88 & 1 & 1 & 10\end{array}$

$\begin{array}{llllllll}\text { P27.3 PG OA } & \text { ET } & \text { DH } & 88 & 10 & 1 & 1\end{array}$

$\begin{array}{llllllll}\text { P27.5 } & \text { PG OA } & \text { ET } & D M & 88 & 1 & 10 & 1\end{array}$

P27.9 PG OA ET DH $83.5 \quad 5.5 \quad 5.5 \quad 5.5$

P28.3 PG OA ET DM $30 \quad 10 \quad 10 \quad 50$

P28.4 PG OA ET DH $40 \begin{array}{lllll}10 & 10 & 40\end{array}$ 
$\begin{array}{lllllllll}\text { P28.5 PG OA ET DM } 50 & 10 & 10 & 30\end{array}$

P28.6 PG OA ET DM $60 \quad 10 \quad 10 \quad 20$

228.9 PG OA ET DM 1107079

$\begin{array}{llllllll}\text { P42.1 PG OA } & \text { ET } D M & 71 & 12.2 & 12.9 & 4\end{array}$

$\begin{array}{llllllll}942.2 & \text { PG OA ET DM } 71 & 12.2 & 12.9 & 4\end{array}$

;

DATA CONSTANT;

MISSING $Q$;

INPUT CODE \$ SOLUB DENSITY;

Cards;

N41.1 $34.5 \quad Q$

N46.9 Q Q

N63 40.3 Q

$\begin{array}{lll}N 78.8 & 73.1 \quad Q\end{array}$

N85.1 $66.0 \quad$ Q

N87.4 10.0 Q

N88.1 Q Q

N88.2 Q $Q$

N12 Q Q

P14 $8.41 \mathrm{Q}$

$\begin{array}{lll}N 43.1 & 7.5 \mathrm{O}\end{array}$

M70_PGO 10.0 Q

M70-PG1 $82.0 \quad 0$

M70-PG2 $58.0 Q$

M70-PG3 $41.0 \quad 0$

M70-PG4 $19.0 \quad Q$

M70-PG5 $9.2 \quad 0$

$M 85 \bar{A} \quad 84^{\circ} Q$

M85B Q Q

$\begin{array}{lll}\text { P5.6 } 19.10 & 0.9577\end{array}$

P8.OA $\quad 0.330 .895$

$\begin{array}{lll}\text { PIOET } 26 & 0.7890\end{array}$

P10.2 $17.00 \mathrm{Q}$

P21.1 Q Q

$\begin{array}{lll}\text { P21.2 } & \text { Q } & \text { Q } \\ \text { P21.3 } & \mathrm{Q} & \mathrm{Q}\end{array}$

P21.4 43.30 1.0677

P21.5 35.001 .1009

$\begin{array}{lll}\mathrm{P} 21.6 & 22.75 \quad 1.0133\end{array}$

P21.7 11.630 .9221

$\begin{array}{lll}\text { P21.8 } & 19.61 & 0.9957\end{array}$

P25.4 15.20 Q

P25.5 23.27 1.0480

$\begin{array}{lll}\text { P25.6 } & 10.52 \quad 0.9738\end{array}$

$\begin{array}{lll}\text { P27.1 } & 8.88 & 1.0381\end{array}$

P27.2 18.111 .0585

P27.3 $8.42 \quad 0.9671$

P27.5 9.410

$\begin{array}{lll}\text { P27.9 } 14.04 & 0.9926\end{array}$

P28.3 $50.59 \quad 1.0664$

P28.4 43.391 .0522

P28.5 $35.77 \quad 1.0348$

P28.6 $27.07 \quad 1.0100$

P28.9 36.400

P42.1 11.370 .9011

;

DATA RESULTS;

MISSING Q;

INPUT CODE $\$$;

DO I=1 TO 4; INPUT SLOPE INCPT @ C; OUTPUT; END;

CARDS;

P21.1 $58.33-186.0151 .26-111.92 \quad 18.24-19.23 \quad 44.16-135.90$ 


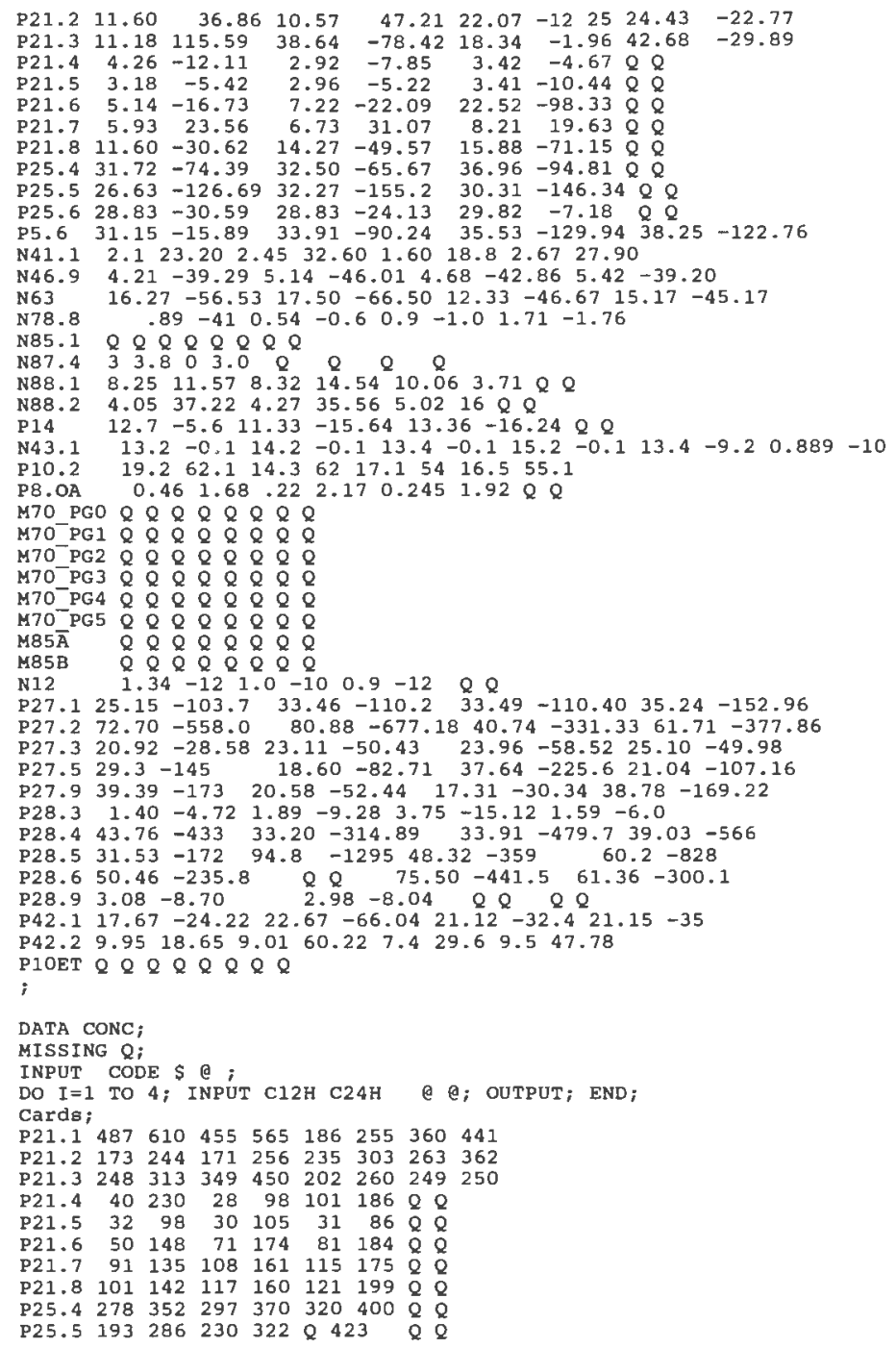




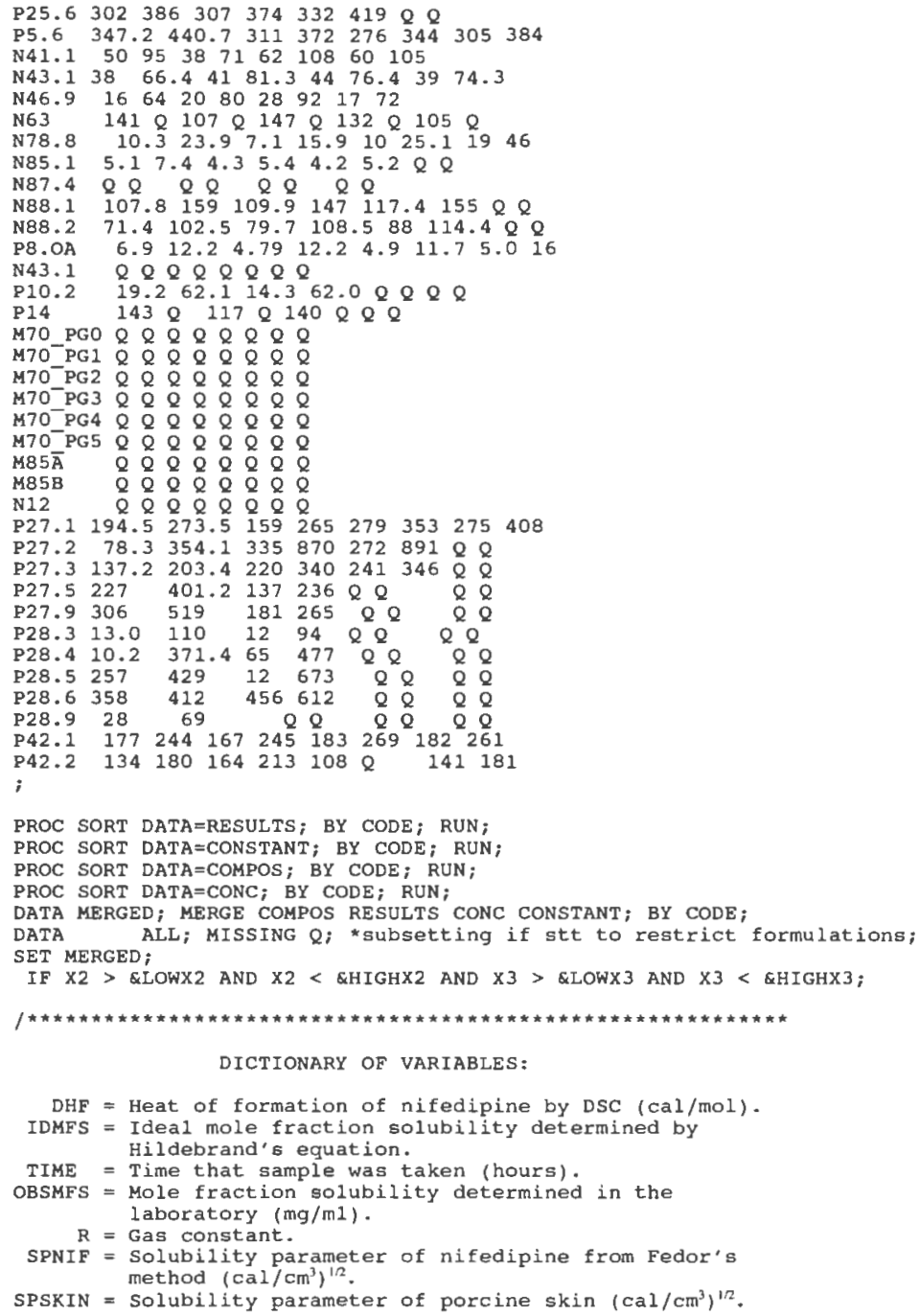




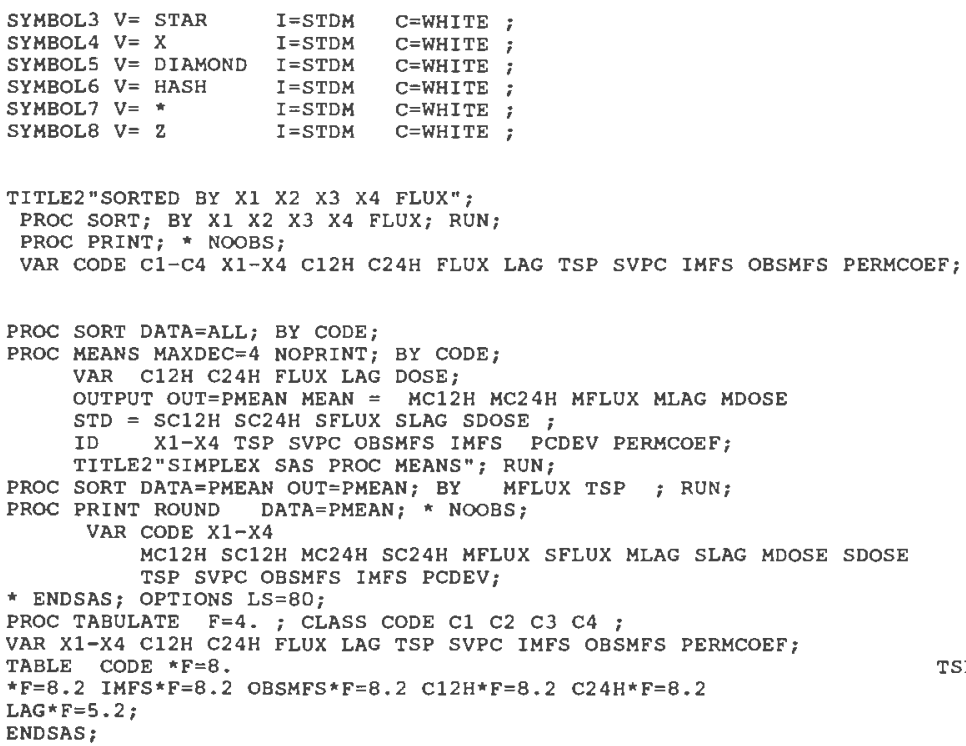




\section{APPENDIX B: \\ NUMERICAL AND STATISTICAL TECHNIQUES \\ INTRODUCTION}

This appendix discusses the rationale behind the attainment of the following objectives: (1) identify the key independent variables which influence the problem and principle responses to be measured; (2) the fitting of some proposed model for the purpose of describing the shape of the response surface over the simplex factor space; and (3) determining the roles played by the individual components. Identification of the right blend of solvents which would allow adequate solubility, stability, permeability with minimal irritancy and lag time at a reasonable cost posed a challenge best solved by the use of appropriate statistical and mathematical tools. These objectives have been achieved through the use of mixture designs. A mixture design is performed by mixing together several ingredients. ${ }^{6}$ Much of the introductory principles of mixture designs, models and the analysis of mixture data may be found primarily in the EXPERIMENTS WITH MIXTURES: Designs, Models, and the Analysis of Mixture Data ${ }^{7}$ from which this appendix draws heavily. In factorial experiments, the response varies depending on the actual amounts of each of the independent variables (i.e., "factors"). However, unlike factorial experiments, the distinguishing feature of a mixture design is that the independent, controllable factors represent proportionate amounts of the mixture rather than unrestrained amounts, the proportions of which must be nonnegative and sum to 
unity. These restrictions limit the manner in which an investigator may: (1) choose the proportions of the ingredients; (2) analyze the data collected from experiments; and (3) interpret the results of the analysis. It is important that experiments be designed so that the independent variables of the system under investigation are perturbed in a controlled, deliberate manner so that observed effects can be properly attributed. For the factorial case, this generally means controlling the factor level combinations. This guideline is not easily applied to mixture problems because a change in one factor demands a commensurate change in the other factor(s). Using a compressed tablet as an example, if the final weight of the tablet were restricted to $500 \mathrm{mg}$, then a $10 \%$ decrease of binder would necessitate a $10 \%$ increase in some other component. It would not be possible to conclude whether change in the tablet's properties are attributable to the decrease in binder or the commensurate increase in other components of the tablet. To avoid this restriction on the factor level combinations, a possible alternative might have been to disperse the enhancers into an inert material (e.g., PEG) and allow the relative mole fraction of PG, OA, ET and DM to vary. This strategy assumes that the filler does not interact with the drug, skin or vehicles which often may not be justified and this approach is a less desirable one. While some materials are not believed to act directly to alter the permeability of stratum comeum lipid, indirect effects, such as alteration of skin hydration may be significant. Information regarding penetration enhancers generally focus on their presumed interaction with the epidermis. Our results suggest that a systematic investigation of these interactions between vehicle component and permeant offer the 
key to explaining nifedipine permeation by comparing the molar ratio of system components to flux.

The number of components is denoted by $q$ and the proportion of the $i^{\text {it }}$ component in the mixture is represented by $x_{i}$, then

$$
x_{i} \geq 0, i=1,2, \ldots, q
$$

and

$$
\sum_{i}^{q} x=x_{1}+x_{2} \ldots+x_{q}=1.0
$$

Since our primary interest is to explain the permeation process from a molecular perspective, the proportionate amounts of mixture component is expressed in mole fraction terms rather than by volume or by weight. The two measured responses of interest (i.e., flux and lag time) are derived from the profiles obtained by plotting the steady state nifedipine permeation as a function of time. The flux is quantified from the slope and the lag time from the point of intersection with the abscissa of the back extrapolated portion of these curves. Due to practical considerations such as cost, irritancy and stability in order to form valid mixtures from which an acceptable formulation could be made, component $i$ must be present in some minimum amount $\mathrm{a}_{i}$ and maximum amount $c_{i}$. In other words, the proportion $x_{i}$ of component $i$ must be bounded below by $a_{i}>0$ and above by $c_{i}<1.0$. The quantity $a_{i}$ is known as the 'lower bound', $c_{i}$ the 'upper bound', and in addition to usual constraints, $x_{i} \geq 0$, $\mathrm{x}_{1}+\mathrm{x}_{2}+\ldots+\mathrm{x}_{\mathrm{q}}=1$, a second limitation for the constrained simplex is

$$
0<\mathrm{a}_{i} \leq \mathrm{x}_{\mathrm{i}} \leq \mathrm{c}_{\mathrm{i}} \leq 1, \mathrm{i}=1,2, \ldots, \mathrm{q}
$$


To be effective the donor must contain relative proportions of the components restricted to the following upper, $\mathrm{c}_{\mathrm{i}}$, and lower, $\mathrm{a}_{\mathrm{i}}$, bounds:

\begin{tabular}{ll}
\hline \multicolumn{2}{c}{ Component Constraints } \\
\multicolumn{1}{l}{ Initial $^{\mathrm{a}}$} \\
\hline Refined
\end{tabular}

The experimental region or factor space of mixture experiments is different than that of factorial experiments. Mixture designs are referred to as a simplex designs because the experimental region of interest, defined by the values ascribed to $x_{i}$, take on the shape of a simplex (i.e., "a regular figure of $n+1$ vertices in $n$ space"). In contrast, for a $2^{q}$ factorial experiment, where $x_{i}$ take on the values of \pm 1 , the factor space is a cuboidal, q-dimensional space. Thus, for a $2^{4}$ factorial, this space is a tetrahedron while for a $2^{4}$ mixture it is a pyramid. The experimental design space constitutes a polyhedron whose eight vertices (points 1-8) are based on the component restrictions mentioned above. The simplex coordinate system for $q=3$ can be plotted on triangular graph paper; graphical representation of $q>3$ is less straightforward. Three methods introduced for locating the vertices of a constrained region as well as for defining some of the centroids of the boundaries are the Saxena Nigram transformation ${ }^{8}$, the McLean Anderson ${ }^{9}$ algorithm and the XVERT algorithm 
developed by Snee and Marquardt ${ }^{10}$. For the present case, the vertices and face and total centroids are established with the extreme vertices algorithm. The two-dimensional faces are found by grouping the vertices of the polyhedron into groups of three of more vertices where each vertex has the same value $x_{i}$ for one of the components. The overall centroid, which is defined as the average of the eight vertices, is listed as point (9). Taken together, the design points comprise the vertices and centroids of the faces, sides and edges of a convex polyhedron compatible with the requirements outlined by Snee ${ }^{11}$ for fitting first-degree and quadratic models. The resulting factor space and the design point designations are presented in Figure 16. The dimensions of a $\{q, m\}$ simplex-lattice must conform to the number of components $(q)$ and the order $(m)$ of the fitted model. The number of design points, $\mathrm{n}$, is

$$
n=\frac{(q+m-1) !}{m !(q-1) !}
$$

Therefore, a quadratic model fitted to the data obtained from the four-component formulations is designated a $\{4,2\}$ simplex and requires at least ten well chosen points, a cubic model a minimum of 20 points, and a quartic model 35 points. The number of terms in the canonical polynomials for these four component formulations would be $4,10,14$, and 20 for a linear, quadratic, special cubic, and full cubic, respectively. These requirements are enumerated here to illustrate how rapidly such designs can escalate. Obviously, a compromise must be struck between accurate modeling of nifedipine permeation and restricting the study to manageable 
proportions. This investigation utilized a $\{4,2\}$ design to delimit the study, so that useful results could be obtained with the time and funds available. which is the special simplex-centroid model of equation (6) when $q=4$. In trying to decide on the particular form of the Scheffe's-type canonical polynomial model to be fitted to data collected at the points of a $q=4$ simplex-centroid design, it is noted that the special cubic model or simplex-centroid model of equation (6) is chosen over the lower-degree models because the terms in the special cubic model not only provide a measure of each pure blend, but provide measures of the binary and ternary blends. Rather than sequentially build the model by starting with the first-degree polynomial and work towards the special cubic model, we began with the complete special cubic model fitted to the data and work backward by testing the usefulness of the cubic term, the quadratic term, the crossproduct terms and finally the similarity of the linear terms in the model. The test statistics are those discussed by Marquardt and Snee (1974) Consequently, the following questions are posed:

1. Is the flux and lag time response surfaces likely to be planar over the experimental space or are combinations of cosolvents likely to cause departures from linearity in the surface shape? If the blending of multiple-components is not additive, which pairs are likely to have synergistic or antagonistic effects? Are complete (quaternary) blends likely to have more desirable responses than temary, binary or even pure blends? Also, if we assume the relationship between components is linear, should the permeation results be collected at vertices of the pyramid only, or should we use 
complete mixtures that are very close to the vertices? Furthermore, even if the relationship is linear, should midledge or interior points be collected to check the assumption of linearity?

2. If the simplex arrangement chosen for the distinct possibility that the surface is not planar, should additional observations be collected at interior points of the polyhedron for the purpose of checking the fit inside the triangle? If so, is the centroid of the triangle the location at which to sample the interior?

3. Are there combinations which yield acceptable flux and lag times? (An acceptable flux must exceed $30 \mathrm{mcg} / \mathrm{h} \cdot \mathrm{cm}^{2}$ and an acceptable lag time must not exceed 4 hours) Initially it is assumed that there exists some fundamental relationship

$$
\eta=\Phi_{\left(x_{1}, x_{2} \ldots, x_{Q}\right)}
$$

which describes the dependence of the response on the proportions, $x_{1}, x_{2}, \ldots, x_{q}$ of the components. The response function in equation (4) is expressible as a low-degree polynomial such as the first-degree polynomial

$$
\eta=\beta_{0}+\sum_{i}^{x} \beta
$$

or the second degree polynomial

$$
\eta=\beta_{0}+\sum_{i}^{n} \beta_{x}+\sum_{i}^{n} \beta_{x_{i}} \beta_{x_{j}}
$$

Besides the difference in their experimental regions, mixture experiments differ from the ordinary regression problems in the form of the polynomial. The canonical form is derived by applying to equation (4) the restriction $\left\{x_{1}+x_{2} \ldots+x_{q}=1.0\right\}$ to the terms of the standard polynomial and simplifying. The reparamatized forms of these 
general polynomials are used because the $x_{i}$ 's are constrained to sum to one and standard computer regression programs cannot be used for fitting the models when the $\mathbf{x}_{i}$ 's sum to unity without creating problems in the calculations.

Terms of higher degree must be added to account for curvature in one or more directions. Quadratic and cubic models with interaction terms was employed. As mentioned previously, the number of terms and thus the number of coefficients increases rapidly with increasing number of factors and increasing order, but fortunately interactions between more than two factors in practice are seldom significant. For the quaternary systems, the number of coefficients required in the polynomial model would be 5, 15 and 35 for a first, second and third degree polynomial, respectively. Polynomial models are not the only model when theoretical grounds suggest another model would be plausible, e.g., a nonlinear model.

A polynomial of fairly high degree would usually be needed to represent the response surface adequately over the whole experimental region and would require an excessive number of experiments. However, it is difficult to translate the physical meaning of quadratic or cubed effects, subsequent permeation experiments (i.e., Design II) were designed so that: (1) complete mixtures be used that are close to the vertices and (2) midledge or interior points be collected to check the assumption of linearity. Midledge observations are advantageous in the sense that if a second degree model is required, the estimates of the coefficients will have smaller variances than if interior points were used to estimate the $\beta$ 's. Inclusion of interior points might be of greater benefit in characterizing synergism among components since interior 
points represent complete blends consisting of all the components simultaneously whereas the edge or boundary points consist of binary mixtures. These observations gave rise to Design II, in which the upper constraint on oleic acid and ethanol was decreased to $10 \%$ mole fraction.

For the final optimization studies, cosolvent blends were formulated by weighing propylene glycol $\left(x_{1}\right)$, cis-oleic acid $\left(x_{2}\right)$, ethanol $\left(x_{3}\right)$, and dimethyl isosorbide $\left(\mathrm{x}_{4}\right)$. Another property of the mixture problem is that the measured response is assumed to depend only on the proportions of the ingredients present in mixture and does not depend on the amount of the mixture. This assumption does not appear to hold and for this reason, the same amount (i.e., moles) of donor were used on all cells. Thus, equimolar doses were applied to the membrane surfaces and the penetration of nifedipine as a function of time was determined, the flux and lag times determined, and the unknown coefficients in the model are determined by the method of least squares. The coefficient estimates are then substituted into the model for use in predicting response values.

Data analysis - Experimental design and analysis is performed using the ADX menu system of SAS/QC, SAS/STAT and SAS/GRAPH software. Although the factors are only observed at a finite number of values, the factors are treated as continuous variables. Using the ranges of each factor, we interpolate over their composite range in order to optimize the responses.

\section{RESULTS}

The functional form of equation (6) of the relationship between the responses 
(flux and lag time) and the factors are given in Table XIII on page 110. The results of a ridge analysis are used to locate the optimum response within the design space (Table XV). This technique computes the estimated ridge of optimum responses for increasing radii from the center of the original design. The optimum values start at the center point and follow the path of steepest descent of ascent, respectively until within the region of experimentation the expected maximum and minimum are found. These results suggest that a relatively high level of PG and low levels of OA, ET and DM will give maximum flux and minimum lag times. Note, however, that the standard error increases as the optimum response ridge moves away from the center point of the design and toward the boundary of the design space, indicating that predictions near the edge are inherently less reliable.

The ridge analysis output is only a list of numbers; to better understand it, consider a plot of the ridge values superimposed on the factor-response profile plot for each factor. Each profile plot is simply a scatter plot of one factor's values against response predictions made over the entire region of experimentation. A profile plot thus shows the relationship of the factor to the response, as well as giving an indication of interactions with other factors. Superimposing the ridge analysis gives an indication of the movement and flow of the optimal response ridge over the multidimensional response surface. When sorted, it becomes obvious that the two objectives, maximizing flux while minimizing flux, conflict with one another. One solution is to set constraints on lag time that it lie below and to optimize flux subject to this constraint. Algorithms are available that permit several responses to be 
simultaneously analyzed to identify mixtures that strike compromises between maximization of some response (e.g. flux) and minimization of another (e.g. lag time). ${ }^{12}$ This useful feature is also incorporated into the SAS ADX menu system.

It is not possible to picture response surfaces of a cubic model because they are hyperplanes in four-dimensional space. The eigenvalues provide additional information about the sensitivity of the stationary point to deviations from the optimum. The relative magnitude of the eigenvalues indicate the relative rate of change for each of the canonical direction $w_{i}$. Those with the largest magnitude change quickest and it is these directions that are the most sensitive to deviation from the actual stationary point. The smaller eigenvalues indicate direction of lesser sensitivity. Unequal eigenvalues suggests that the behavior of the response function results in contours that are elliptical. The major axis belongs to the least sensitive direction (given by an eigenvector) and the minor axis is that of the greater eigenvalue. This ridge analysis provides further knowledge about which directions must be estimated most precisely and which are less important.

The crossproduct coefficient estimates (the $\beta_{i j}$ 's) measure curvilinear departure from a hypothetically planar response surface. The synergistic blending of $O A$ appears to be real since all $\beta_{\mathrm{ij}}$ for $\mathrm{i}=\mathrm{j}=2$ terms are greater than zero. Synergism among the other components may not be real, since $\beta_{i j}$ is not significantly greater than zero based on the comparison of each coefficient estimate to the value of its standard error in the form of an approximate Student's t-statistic and then comparing each computed value of $t$ to the tabled value $t_{15.0 .25}$ The sign of the $\beta$ 's and the 
corresponding value of the t-statistic lead us to infer that the individual terms are antagonistic toward one another in overall formulation performance while combinations of factor 2 (i.e., oleic acid) strongly interacts with all three factors. Furthermore, the magnitude of the interaction terms are large and probably represents the driving force for enhanced permeability in these systems. 


\section{REFERENCES}

1.Shapiro, S.S.and J.A. Comell, eds. The ASOC Basic References in Quality Control: Statistical Techniques, Vol. 5 "How to Run Mixture Experiments for Product Quality." Milwaukee, Wisconsin 1983.

2.Comell, J.A. EXPERIMENTS WITH MIXTURES: Designs, Models, and the Analysis of Mixture Data New York: John Wiley \& Sons 1983.

3.Saxena, N.K.; Nigram, A.K. "Restricted Exploration of Mixtures by Symmetric Simplex Designs." Technometrics 1977, 19, 47-52.

4.McLean, R.A.; Anderson, V.L. "Extreme vertices design of mixture experiments." Technometrics $1966,8,447-454$.

5.Snee, R.D.; Marquardt, D.W. "Extreme Vertices Designs for Linear Mixture Models." Technometrics 1974, 16, 399-408.

6.Snee, R.D. "Experimental Designs for Quadratic Models in Constrained Mixture Spaces." Technometrics 1975, 17, 517-528.

7.Belloto, R.J.; Dean, A.M.; Moustafa, M.A.; Molokhia, A.M.; Gouda, M.W.; Sokolski, T.D. "Statistical Techniques Applied to Solubility Predictions and Pharmaceutical Formulations: An Approach to Problem Solving Using Mixture Response Surface Methodology." International Joumal of Pharmacy 1985, 23, 195-207. 


\section{APPENDIX C:}

\section{PROTOTYPE TRANSDERMAL DEVICE DESIGN}

INTRODUCTION - The effect of adhesive composition on TDS performance is well recognized. In previous studies, we have investigated the role of solubility and the influence of formulation components and permeation enhancers on the skin permeability of the very hydrophobic calcium channel blocker nifedipine. Rate and extent of nifedipine permeation was enhanced in vitro by the judicious choice of donor solvent vehicle composition. The total amount of drug absorbed, Q, was found to be directly related to the volume of propylene glycol (PG), ethanol (ET) and dimethyl isosorbide (DM). The literature has noted that $\mathrm{ET}^{1}$ and $\mathrm{PG}^{2}$ are well absorbed when applied topically and our work has demonstrated that the permeation of nifedipine may be linked to the presence of these cosolvents in our formulations. Investigations such as those performed by $\operatorname{Lin}^{3}$ demonstrate that cosolvent levels are likely to significantly affect the mechanical properties of the device, especially at the skin:device interface. Preliminary results have shown that the delivery of clinically useful amounts of nifedipine may require relatively large amounts of solvent at the skin-device interface. This report examines the effect of two donor formulations on the adhesive properties and drug release from a variety of pressure sensitive adhesive (PSA) films.

Frequently, the fabrication of PSA's require heat to assist crosslinking and to 
drive off residual solvent. ${ }^{4}$ The deleterious effect of high temperatures required by solvent-based and hot-melt coating processes pose limitations on the selection of enhancers such that avoidance of a heating step would be useful. Low temperature curing of silicone rubber to prepare a monolithic TDS would be particularly useful when enhancers of low vapor pressure are incorporated into the formulation. This study evaluates silicone elastomer matrices containing drug and penetration enhancer fabricated by polymerizing silicone precursors at room temperature. Alternatively, steps that require processing at elevated temperatures may be executed prior to the addition of heat-sensitive components. This was accomplished by the formation of PSA films and their subsequent lamination, at room temperature, to a layer incorporating the drug and enhancers. Matrix and reservoir-type devices thus formed are evaluated to deliver drug and enhancer to the skin.

EXPERIMENTAL - All materials were gifts of the respective companies unless noted otherwise and were used as received. Ethanol, isopropyl myristate, nifedipine, oleic acid and propylene glycol were purchased from Sigma Chemical Co. St Louis, MO 63178. Azone (Whitby Research, 2801 Reserve Street, Richmond, VA 23261), dimethyl isosorbide (ICI Specialty Chemicals, Wilmington, DE 19897), ethylene vinyl acetate (EVA) from $3 \mathrm{M}$ and silicones from Dow-Corning were gifts. The cosolvent vehicle was composed of oleic acid, ethanol, and propylene glycol (mole fraction per cent $1: 2: 7$ ). Stock drug:enhancer suspensions (10\% excess nifedipine) were prepared for subsequent manufacture of devices. 
Polymers and resins - The polymer substrates, being dispersions of monomers in a volatile carrier, are expressed in terms of weight of solids. The monolithic silicone rubber-enhancer-drug matrix (SE) consisted of a two part 360:382 Silicone Medical Fluid and a Silastic MDX4-4210 Medical Grade Elastomer (Dow Coming Corp., 2020 Willard H. Dow Center Midland, MI 48674). The pressure sensitive adhesives (PSA) evaluated were: (1) silicone (SA) X7-4301 and X7-2920 (Dow Coming); (2) polystyrene-block-polyisoprene-block-polystyrene (PIB) of 15:85 styrene/rubber ratio (Shell Schweiz AG, CH Zurich); (3) polyacrylic DURO-TAK 120-1753 (PA) crosslinked with $4 \%$ w/w aluminum acetyl acetonitrile (National Starch and Chemical Company Bridgewater, NJ); and (4) ethyl:vinyl acetate hot melt (EVA) (33:400/28:800). The hot-melt mixtures were modified with Hercules C, Abitol and Cetiosol S (Scheller AG, CH-Zurich) and heated to $70-80^{\circ} \mathrm{C}$.

Monolithic matrix preparation - The monolithic silicone devices were prepared from the 360:382 Medical Fluids or the Silastic MDX4-4210 silicone elastomer by thoroughly mixing one part of curing agent with 10 parts by weight of the base elastomer. After five minutes of blending in a rotary mixer (Electrocraft Co, 1600 Second Street Hopkins, MI) the desired weight percent of drug and enhancer are added incrementally to the silicone mixture. The mixture is then drawn up into a 20 $\mathrm{ml}$ syringe and cured for 72 hours at room temperature to permit complexation and crosslinking. The cured polymeric cylinder is sliced into $0.5 \mathrm{~cm}$ thick slices. PSA film preparation - The stock liquid drug:enhancer mixtures were added incrementally to the liquid PSA's in a ratios of $1: 1: 8$, mixed for 10 minutes and then 
applied to polyester ( $\mathrm{PE}$ ) backing layers (3M Company). The films were prepared in thicknesses of 50 and 200 microns in a suspended-knife apparatus. Removal of residual solvent was accomplished by evaporation in a ventilated oven set at $60^{\circ} \mathrm{C}$ for 20 minutes. The films were then laminated onto a foil release liner and stored under ambient conditions.

Reservoir device preparation - Polypropylene (PP) membrane of $38 \%$ porosity (Celgard 2400 Celanese Separations Products Box 32414, Charlotte, N.C. 28232) was investigated as retaining membrane. Scotchpack heatsealable, polyester (PE) film laminate (No. 1022 3M Company) comprised the backing layer. Silicone PSA films were applied to the release liner (PE film No.9747, 3M Company), a layer of Celgard placed atop the PSA and the backing layer placed atop the Celgard layer. The laminate thus formed was heat sealed using a die compressed for 13 seconds at $70^{\circ} \mathrm{C}$ to form a patch with a reservoir between the Celgard and backing layer (see Figure 27). The patch reservoir was loaded by inserting a hypodermic syringe into the orifice leading into the reservoir chamber, expressing $0.3 \mathrm{ml}$ of the stock drug:cosolvent mixtures containing $10 \%$ excess nifedipine into the chamber and heat sealing the orifice. Patches were stored for one week prior to testing to allow the reservoir components to equilibrate with the PSA.

Partioning studies - An equilibrium method ${ }^{5}$ was used to determine the membrane-vehicle partition coefficient. Medical grade silicone adhesive was cast into membranes, ground into small pieces, weighed and placed into screw top test tubes. A known volume of 0.01 molar phosphate buffered nifedipine solutions was added to 


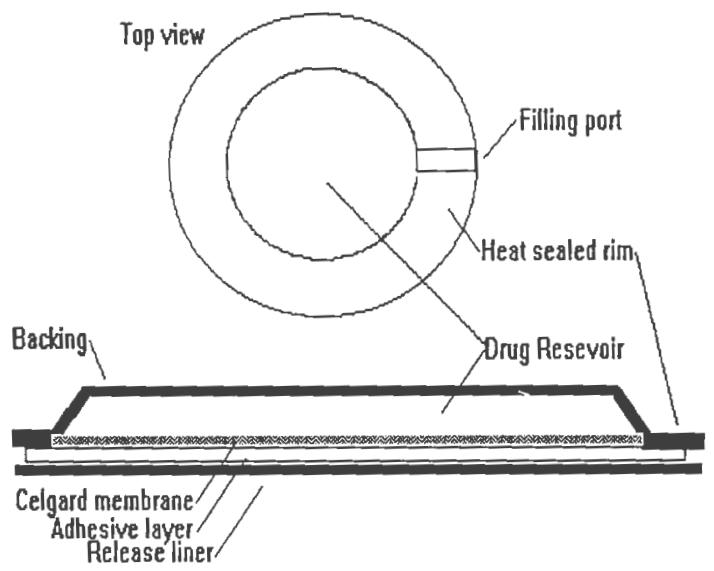

Figure 27. Schematic of prototype resevoir transdermal delivery system.

each tube and placed in a shaker bath held at $37^{\circ} \mathrm{C}$ for 2 weeks. For each sample vehicle studied, several tubes were prepared, samples were withdrawn at daily intervals, and the resulting concentrations were determined. For all cases, equilibrium was reached within 5 days as evidenced by no further decrease in the solution concentration. The partition coefficient was calculated from the change in the nifedipine concentration of the solutions before and after the partition. The concentration of the drug was measured by HPLC. For the purpose of calculation, the density of the dimethylsiloxane was assumed to be unity, which is a reasonable approximation. ${ }^{6}$ The partition coefficient was calculated by dividing the membrane solubility by the saturation solubility in the solvent system under consideration. Total 
amount recoverable was determined by placing membranes in $30 \mathrm{ml}$ scintillation vials and agitated at 30 oscillations per minute at $37^{\circ} \mathrm{C}$. The fluid in the vials was replaced until no more nifedipine was detected.

Kinetic studies - Screening of PSA films were carried out by measurement of penetration rates through freshly excised hairless mouse skins mounted in Franz cells to measure their usefulness as delivery devices. The laminates were placed on the excised skin, a 200 gram weight placed atop for 2 minutes, and then mounted in the Franz cell.

RESULTS - The physical properties of the films studied and permeation of nifedipine through the materials tested in this initial screening are shown in Table XVI. No decomposition of nifedipine could be detected by HPLC, suggesting that the patch components were resistant to oxidation and did not need stabilizing additives often necessary in many organic-based PSAs. In addition to the numerous advantages reported for silicone-based devices, ${ }^{7,829}$ the silicone PSA tested appears to retain a suitable degree of tack for initial bonding, conformance to skin contours and accommodation of skin movement needed to hold the TDS in place for several days, sufficient cohesive strength is observed leaving no residue when removed.

Physical compatibility when measured by the 180 degree peel test showed the adhesive strength of the $200 \mu$ films were affected in the following order: SISPSA $<$ SPSA < PPSA < HMPSA. The results in Table XVI show good physical stability in silicone PSA. To our knowledge, there is but one report evaluating nifedipine:PSA 
Table XVI. Properties of acrylic, rubber-based and silicone matrix and pressure sensitive adhesives.

\begin{tabular}{|l|l|l|}
\hline POLYMER & Tack & Peel \\
\hline DURO-TAK 120-1753 & high & high \\
\hline Ethylene vinyl acetate & low & low \\
\hline PIB 15:85 & medium & medium \\
\hline Silicone Matrix 360:382 & none & none \\
\hline $\begin{array}{l}\text { Silicone elastomer } \\
\text { X7-4301 }\end{array}$ & high & medium \\
\hline
\end{tabular}

compatibility and this was performed at a $1 \%$ loading level. ${ }^{10}$ Best performance was obtained from a TDS design composed of four basic components: an impermeable backing, an adhesive, a drug reservoir, and microporous membrane to contain the drug suspended in vehicle. The amount of nifedipine permeated as a function of time for the silicone reservoir device is shown in Figure 28. These results demonstrate that, in terms of nifedipine permeation rates and physical properties, silicone is the best choice of the PSA's examined. The range of drugs that may be formulated as TDS's, particularly drugs whose permeation profiles are linked to the codiffusion of vehicle, may be extended by the adoption of reservoir-type designs.

In summary, the in-vitro skin permeation profiles of nifedipine $(\mathrm{N})$ under the influence of selected penetration enhancers from adhesive matrix devices was 


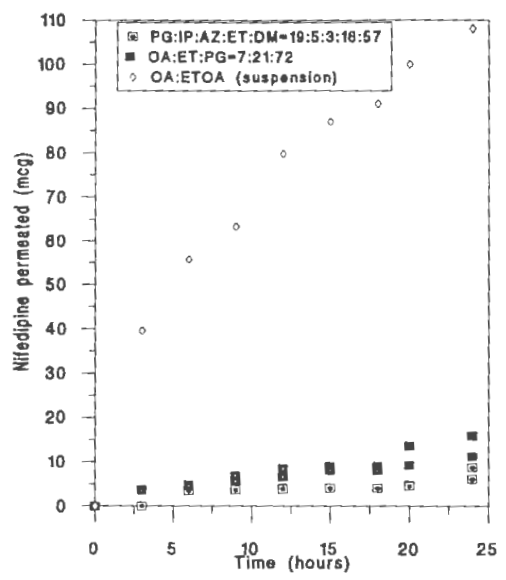

evaluated using excised hairless mouse skin. Prototype devices containing permeation enhancers were prepared from silicone adhesives, silicone elastomer, pressure sensitive acrylic, and PIB pressure sensitive adhesives (PSA). Release from thin films of these materials applied directly to the skin was poor. Fluxes were substantially higher when the drug:cosolvent mixture was fabricated as a reservoir system. 


\section{REFERENCES}

1. Liu, Purchum, Tamie Kurihara-Bergstrom, and William R. Good, "Cotransport of Estradiol and Ethanol Through Human Skin In Vitro: Understanding the Permeant/Enhancer Flux Relationship." Pharmaceutical Research 8 (July 1991): 938-944.

2. Mollgaard, Birgitte and Hoelgaard, Annie. "Vehicle Effect on Topical Drug Delivery II. Concurrent Skin Transport of Drugs and Vehicle Components." Acta Pharm Suec. $1983,20,443-450$.

3. Lin SY "The Effect of Plasticizers on Compatibility, Mechanical Properties and Adhesion Strength of Drug-free Eudragit E Films." Pharm, Res . 1991, 8 119-49.

4. Satas,D.(Ed.), Handbook of Pressure Sensitive Adhesive Technology, 2nd edition, Van Nostrand Reinhold, New York, 1989.

5. Scheuplein, R. J. Invest. Dermatol, 45: 334-336 1965.

6. Addicks, William I., G.L. Flynn, N. Wiener, and C. Chiang, Topical drug delivery from thin applications: theoretical predictions and experimental results. Pharm. Res. 7:10 $1048(1990)$.

7. Thomas, S., and Pfister, W.R., "The Emerging Role of Silicones Used in Transdermal Drug Delivery Systems." S.T.P. 1 (1991): 38-46.

8. Pfister, W.R., "Silicone Adhesives for Trandermal Drug Delivery Systems." DCI October (1988) 44-52.

9. Sheth, N.V., Freeman, D.J. , Higuchi, W.I. and Spruance, S.L. The Influence of Azone, Propylene Glycol and Polyethylene Glycol on In-Vitro Skin Penetration of Triflurothymidine. Intemational Joumal of Pharmaceutics 28 (July 1986): 201-209.

10. Pfister, William R. "Silicone Adhesives for Transdermal Drug Delivery Systems". Drug and Cosmetics Industry (October 1988): 44-52. 


\section{APPENDIX D}

\section{CONCLUSIONS AND SUGGESTIONS FOR FUTURE WORK}

The ultimate goal of this type of research is not restricted to merely overcoming the formidable barrier posed by the skin but rather is an attempt to avoid the empirical approach in favor of quantitative, scientific tactics. We have achieved the former and made significant progress with the latter. Admittedly, extrapolation of the results of our inquiries to other systems is a dangerous exercise. More often than not, conclusions are merely assumptions to be tentatively entertained. Yet the decision process behind the choice of the enhancer-solvent combinations for other hydrophobic penetrants having different physicochemical properties is a topic worthy of further consideration. If our hypotheses regarding the crucial effect of solubilization within the epidermal layers is correct and if our observations were mirrored by other permeants, inferences made early in the development process regarding TDS design could be justified.

Foremost in our minds at this time is the disposition of non-drug components that constitute the donor. The data presented constitutes a strong case for a hypothetical linkage of solvent permeation with drug permeation and their measurement holds the key to the interpretation of the information we have gathered. Furthermore, measurements should be conducted to determine skin uptake of enhancers e.g., oleic acid because it may be that enhancer skin uptake, hence 
penetration enhancement, is dependent on thermodynamic activity of the oleic acid in vehicle rather than that of the drug. Sheets of epidermis, including the horny layer, may be separated from the underlying dermis and mounted in diffusion cells so that the diffusion and preferential uptake of compounds may be followed. Future work might utilize these techniques in the hope of correlating flux through intact skin to flux through the perturbed membrane, thus identifying the site of control.

The techniques used in this thesis were subject to constraints imposed by equipment; alternative strategies could have been employed to examine the effect of cosolvent composition on permeation. Although we employed an infinite dose design with regard to drug, the results clearly display a dependence of permeation on the molar amount of cosolvent applied and future work might take this into consideration. Radiolabeled drug and cosolvent would permit direct quantitation. Unfortunately, radiolabeled nifedipine and dimethylisosorbide were unavailable for use in these experiments; methods that permit direct quantitative measurements of drug and enhancer in the skin compartment should be considered. The potential of microdialysis, a new bioanalytical sampling technique that permits dermal flux of topically applied compounds to be monitored directly, has proved to be a powerful technique in the characterization of dermal transport of 5-FU. ${ }^{1}$

Finally, a need exists to develop methods to predict the in vivo performance of TDSs. Such methods would be invaluable in engineering new systems. As we increase our understanding of how dosage form components interact with the skin we likewise increase our proximity to this elusive goal. 


\section{REFERENCES}

1. Auldt, J.M.; Lunte, C.E.; Meltzer, N.M.; Riley, C.M. "Microdialysis Sampling for the Investigation of Dermal Drug Transport." Pharmaceutical Research 1992, 9, 1256-1261. 


\section{BIBLIOGRAPHY}

Addicks, William J., G.L. Flynn, N. Wiener, and C. Chiang. "Topical Drug Delivery from Thin Applications: Theoretical Predictions and Experimental Results." Pharmaceutical Research 7 (1990): 1048-52.

Anderson, Bradley, D., J. Howard Rytting and Takeru Higuchi. "Solubility of Polar Organic Solutes in Nonaqueous Systems:Role of Specific Interactions. " Journal of Pharmaceutical Sciences 69 (July 1980): 770-2.

Auldt, Joseph M., Craig E. Lunte, Noel M. Meltzer, and Christopher M. Riley. "Microdialysis Sampling for the Investigation of Dermal Drug Transport." Pharmaceutical Research 9 (October 1992): 1256-61.

Ali, Syed Laik. "Nifedipine." In Klaus Florey, ed. Analytical Profiles of Drug Substances. Vol. 18, San Diego: Academic Press, Inc., 1983.

Barry B.W. "Mode of Action of Penetration Enhancers in Human Skin." Journal of Controlled Release, 6 (1983): 85-97.

Barry B.W., S.M. Harrison, and P.H. Dugard. "Vapor and Liquid Diffusion of Model Penetrants Through Human Skin: Correlation with Thermodynamic Activity." Journal of Pharmacy and Pharmacology 37 (1985): 226-36.

Barton, Alan F.M. ed. In Handbook of Solubility Parameters and Other Cohesion Parameters. Boca Raton: CRC Press, Inc., 1990.

Belloto RJ, A.M. Dean, M.A. Moustafa, A.M. Molokhia, M.W. Gouda and T.D. Sokolski. "Statistical Techniques Applied to Solubility Predictions and Pharmaceutical Formulations: An Approach to Problem Solving Using Mixture Response Surface Methodology." International Journal of Pharmacy 23 (1985): 195-207.

Bennett, S.L., B.W. Barry and R. Woodford. "Optimization of Bioavailability of Topical Steroids: Nonoccluded Penetration Enhancers Under Thermodynamic Control." Journal of Pharmacy and Pharmacology. 37 (1985): 298-304.

Berner, Bret, Gerard C. Mazzenga, John H. Otte, Robert J. Steffens, Rong-Hwei Juang, and Charles D. Ebert. "Ethanol:Water Mutally Enhanced Transdermal Therapeutic System. II. Skin Permeation of Ethanol and Nitroglycerin." Joumal of Pharmaceutical Sciences 78 (1989): 402-7.

Brown, Leonard and Robert Langer. "Transdermal Delivery of Drugs." Annual Review of Medicine 39 (1988): 221-9. 
Barry, W. "Modern Methods of Promoting Drug Absorption Through the Skin." Molecular Aspects of Medicine 12 (March 1991): 195-241.

Calis, Karim Anton, David R. Kohler, and Diane M. Corso. "Transdermally Administered Fentanyl for Pain Management." Clinical Pharmacy 11 (January 1992): 22-36.

Chien, Yie W., Heliang Xu, Chin-Chih Chiang, and Yih-Chain Huang. "Transdermal Controlled Administration of Indomethacin. I. Enhancement of Skin Permeability." Pharmaceutical Research 5 (February, 1988): 103-6.

Chung, Menger, Donald P. Reitberg, Michael Gaffney, and Walter Singleton. "Clinical Pharmacokinetics of Nifedipine Gastrointestinal Therapeutic System. A Controlled-release Formulation of Nifedipine." American Journal of Medicine 83 Suppl 6B (December 1987) 10-14.

Comfort, Ann, Steven M. Dinh, John Otte, Ihor Shevchuk, and Bret Bemer. "Enhanced Transport in a Therapeutic Transdermal System." Biomaterials 11 (November 1990): 729-33.

Comell, John A. EXPERIMENTS WITH MIXTURES: Designs, Models, and the Analysis of Mixture Data. New York: John Wiley \& Sons 1983.

Crotin, C.M. "Nifedipine Pharmocokinetics." Drug Intelligence and Clinical Pharmacy 21 (June 1987): 642-4.

Dean, John A. Lange's Handbook of Chemistry. 13th ed. New York: McGraw-Hill Book Company 1985.

Diem, K. and C. Letner. Documenta Geigy Scientific Tables. 7th ed. Basel Switzerland. 1970 p. 604.

Diez, I., H. Colom, J. Moreno, R. Obach, C. Peraire, and J. Domenech. "A Comparative In Vitro Study of Transdermal Absorption of a Series of Calcium Channel Antagonists." Journal of Pharmaceutical Sciences 80 (October, 1991): 931-4.

Dow Chemical Company, Performance Products Department, Polyglycols Technical Sevices and Development B1605, 2301 N. Brazosport Blvd, Freeport, TX 77541 3257. Trudy Wells, personal communication (409) 238-9284.

Dunn, William J., John H. Block and Robert S. Pearlman. Partition Coefficient: Determination and Estimation. Pergamon Press, Elmsford, New York 1986. 
Fedors, Robert. "A Method for Estimating Both the Solubility Parameters and Molar Volumes of Liquids." Polymer Engineering and Science 14 (February 1974):

147-154.

Fedors, Robert. "A Method for Estimating Both the Solubility Parameters and Molar Volumes of Liquids." Polymer Engineering and Science. 14 (February 1974): 147-154.

Grant, David J.W. and Takeru Higuchi, eds. "Solubility Behavior of Organic Compounds: Origin of Molecular Interactions." Techniques in Chemistry Vol. 22 by Gordon Flynn. New York: John Wiley \& Sons, 1990.

Flynn, Gordon L. and B. Stewart. "Percutaneous Drug Penetration: Choosing Candidates for Transdermal Development. "Drug Development in Pharmaceutical Research 13 (1988): 169-85.

Francoeur, Michael L., Gula M. Golden, and Russell O. Potts. "Oleic Acid: Its Effects on Stratum Corneum in Relation to (Trans)Dermal Drug Delivery." Pharmaceutical Research 7 (June 1990): 621-627.

Friend, David R., Paul Catz, Jorge Heller, and Michael Okagaki. "Transdermal Delivery of Levonorgestrel. IV: Evaluation of Membranes." Journal of Pharmaceutical Sciences 78 (June 1989): 477-80.

Friend D.R. "Cutaneous Effect of Transdermal Levonorgestrel." Food and Chemical Toxicology 29 (September 1991): 639-48.

Frishman, William H., David Sherman, and Donald A. Feinfeld. "Innovative Drug Delivery Systems in Cardiovascular Medicine." Cardiology Clinics 5 (November 1987): 703-716.

Ghanem, Abdel-Halim, Hanaa Mahmoud, William I. Higuchi, and Yie H. Kim. "Evaluation of the Transport Enhancing Effects of a Series of Alkanols for $\beta$ Estradiol and Other Permeants in Hairless Mouse Skin." Pharmaceutical Research 5 (1988): S119.

Good, William R., Marilou S. Powers, Patricia Campbell and Lotte Schenkel. "A New Transdermal Delivery System for Estradiol." Journal of Controlled Release 2 (1985): 89-97.

Grulke, Eric A. "Solubility Parameters Values VIl/519-59." In J. Brandrup, and E.H. Immergut, eds. Polymer Handbook, 3rd ed. New York: John Wiley \& Sons, 1989.

Guy, Richard H. and Jonathan Hadgraft, eds. "Selection of Drug Candidates for 
Transdermal Drug Delivery: Developmental Issues and Research Initiatives." In Transdermal Delivery Systems New York: Marcel Dekker, Inc., 1989.

Guy, Richard H. and Jonathan Hadgraft. "Selection of Drug Candidates for Transdermal Drug Delivery." In Transdermal Delivery Systems: Developmental Issues and Research Initiatives. New York, N.Y.: Marcel Dekker, 1989. 59-82.

Hadgraft Jonathan. "Penetration Enhancers in Percutaneous Absorption". Pharmacy International, (1984): 252-54.

Hall, Ricard C. "Nifedipine Formulations." Medical Jotrnal of Australia 147 (August 1987): 148-50.

Hansen, Charles M. "The Three Dimensional Solubility Parameter - Key to Paint Component Affinities: II and III." Journal of Paint Technology 39 (1967): 505-17.

Hansen, Charles and Alan Beerblower. "Solubility Parameters." In A. Stenden, ed., Encyclopedia of Chemical Technology. Suppl Vol, 2nd ed. New York: Wiley \& Sons $1972,889-910$.

Hoelgarrd, Annie and Birgitte Mollgaard. "Dermal Drug Delivery-Improvement by Choice of Vehicle or Drug Derivative." Joumal of Controlled Release 2 (1985):11120 .

Hori, Mitsuhiko, Howard Maibach, and Richard Guy. "Enhancement of Propranolol and Diazepam Skin Absorption In Virro. II: Drug, Vehicle, and Enhancer Penetration Kinetics." Journal of Pharmaceutical Sciences 81 (April 1992): 330-33.

Hori, Mitshuhiko and Susumu Satoh. "Classification of Percutaneous Penetration Enhancers: A Conceptual Diagram." In Robert L. Bronaugh and Howard I. Maibach, eds. Percutaneous Absorption Second Edition. New York: Marcel Dekker, Inc., 1992.

Idson, Bernard. "Percutaneous Absorption Enhancers." Drug and Cosmetic Industry (July 1985): 30-33.

Izumoto, T., A. Aioi, S. Uenoyama, K. Kuriyama, and M. Auma. "Relationship Between the Transference of a Drug from a Transdermal Patch and the Physicochemical Properties." Chemical Pharmaceutical Bulletin, 40 (February 1992): 456-58.

Jacobsen P, O. Lederballe Pederson, E. Mickelsen. "Gas Chromatographic Determination of Nifedipine and One of its Metabolites Using Electron-capture Detection." Iournal of Chromatography 162 (1979): 81-87. 
Janssen Pharmaceutica. Duragesic package insert. Piscataway, NJ; January 1991.

Keith, Lawrence H. and Douglas B. Walters. In National Toxicology Program's Chemical Solubility Compendium Chelsea, MI: Lewis Publishers, Inc. 1992.

Khalil, Said A. and Alfred Martin. "Drug Transport through Model Membranes and its Correlation with Solubility Parameters." Journal of Pharmaceutical Sciences 56 (October 1967): 1225-1233.

Kier, Lermont B. "Quantitation of Solvent Polarity Based on Molecular Structure." Journal of Pharmaceutical Sciences 70 (August 1991) 930-33.

Kleinbloesem, C.H., P. van Brummelen, and D.D. Breimer. "Nifedipine. Relationship Between Pharmacokinetics and Pharmacodynamics." Clinical Pharmacokinetics 12 (January 1987): 12-29.

Knutson, K., S.L. Krill, and J. Zhang. "Solvent-mediated Alterations of the Stratum Corneum." Journal of Controlled Release 11 (1990): 93-103.

Kondo, Shuji, H. Yamasaki-Konishi, and Isao Sugimoto. "Enhancement of Transdermal Delivery by Superfluous Thermodynamic Potential. I. Thermodynamic Analysis of Nifedipine Across the Lipoidal Barrier" Journal of Pharmacobiological Dynamics 10 (1987): 587-594.

Kondo, Shuji, H. Yamasaki-Konishi, and Isao Sugimoto. "Enhancement of Transdermal Delivery by Superfluous Thermodynamic Potential. II. In Vitro-In Vivo Correlation of Percutaneous Nifedipine Transport." Journal of Pharmacobiological Dynamics 10 (1987): 662-668.

Kondo, Shuji, Tsunemasa Mizuno, and Isao Sugimoto. "Effects of Penetration Enhancers on Percutaneous Absorption of Nifedipine. Comparison between DEET and Azone." Joumal of Pharmacobiological Dynamics 11 (1988): 88-94.

Krill, K., S.L., Knutsun, and William I. Higuchi. "Ethanol Effects on the Stratum Corneum Lipid Phase Behavior." Biochimica Biophysica et Acta 1112 (December 1992): 281-6.

Kydonieus, Agis F. "Fundamentals of Drug Delivery" In A.F. Kydonieus and B. Berner, eds. Transdermal Drug Delivery, Volume I, Boca Raton: CRC Press, 1990.

Lin ,S.Y. "The Effect of Plasticizers on Compatibility, Mechanical Properties and Adhesion Strength of Drug-Free Eudragit E Films." Pharmaceutical Research 8 (September 1991): 119-29. 
Liron, Zvi and Sasson Cohen. "Percutaneous Absorption of Alkanoic Acids. II: Application of Regular Solution Theory." Journal of Pharmaceutical Sciences 73 (1984): 538-42.

Liu, Purchum, Tamie Kurihara-Bergstrom, and William R. Good. "Cotransport of Estradiol and Ethanol Through Human Skin In Vitro: Understanding the Permeant/Enhancer Flux Relationship." Pharmaceutical Research 8 (July 1991): 93844.

Loftsson, T., N. Gildersleeve, R. Soloman and Nicholas Bodor. "Effect of Oleic Acid on Diffusion of Drugs Through Hairless Mouse Skin." Acta Pharmaceutica Nordica 1:1 (January 1989): 17-29.

Loth, H. "Vehicular Influence on Transdermal Drug Penetration." International Journal of Pharmacy 68 (January 1991): 1-10.

Loth, H. "Skin Permeability." Methods and Findings in Experimental and Clinical Pharmacology 11 (March 1989): 155-64.

Martin, Alfred, James Swarbrick, and Arthur Camarata. Physical Pharmacy. 3rd ed. Philadelphia: Lea \& Febiger 1983.

Martin, Alfred, P.L. Wu and T. Velasquez. "Extended Hildebrand Solubility Approach: Sulfonamides in Binary and Ternary Solvents. " Journal of Pharmaceutical Sciences 74 (March 1985): 277-82.

McLean, R.A. and V.L. Anderson. "Extreme Vertices Design of Mixture Experiments." Technometrics. 8 (1966): 447-54.

Mitsuhiko Hori, Howard Maibach and Richard Guy. "Enhancement of Propranolol and Diazepam Skin Absorption In Vitro. II: Drug, Vehicle, and Enhancer Penetration Kinetics." Joumal of Pharmaceutical Sciences 81 (April 1992): 330-3.

Mollgaard, Birgitte and Annie Hoelgaard. "Vehicle Effect on Topical Drug Delivery: II. Concurrent Skin Transport of Drugs and Vehicle Components." Acta Pharmaceutica Suecica 20 (1983): 443-50.

Morimoto, Kazohiro, Hirobumi Tabata, and Katsuaki Morisaka. "Nasal Absorption of Nifedipine from Gel Preparations in Rats." Chemical Pharmaceutical Bulletin 35 (July 1987): 3041-4.

Mullins, L.J. "Some Physical Mechanisms in Narcosis." Chemical Reviews 54 (1954) 289-323. 
Nomura, H. F. Kaiho, Y. Sugimoto, Y. Miyashita, M. Dohi and Y. Kato,

"Percutaneous Absorption of Indomethacin from Mixtures of Fatty Alcohol and Propylene Glycol (FAPG Bases) Through Rat Skin: Effect of Oleic Acid Added to FAPG Base." Chemical Pharmaceutical Bulletin 38:5 (May 1990): 1421-3.

Dertel, R.P. "Protein Conformation Changes Induced in Human Stratum Comeum by Organic Sulfoxides: an IRS Investigation." Biopolymers 16 (1977): 2329-45.

Okamoto, Hirokazu, Mirsuru Hashida, and Hitoshi Sezaki. "Effect of 1-Alkyl or 1.Alkylazacycloalkanone Derivative on the Penetration of Drugs with Different Lipophilicities through Guinea Pig Skin." Journal of Pharmaceutical Sciences 80 (January 1991): 41-5.

Osbom, David W. "Computational Methods for Predicting Skin Permeability." Pharmaceutical Manufacturing 3 (April 1988): 41-8.

Pasanisi F., P.A. Merideth, and J.L. Reid. "Pharmacokinetics of Nifedipine." International Joumal of Clinical Pharmaceutical Research 1 (January 1985): 63-6.

Pershing, Lynn K., Lyssa D. Lambert, and Kristine Knutson. "Mechanism of Ethanol-Enhanced Estradiol Permeation Across Human Skin in Vivo." Pharmaceutical Research 7 (February 1990): 170-5.

Pfister, William R., "Silicone Adhesives for Trandermal Drug Delivery Systems. II: System Design Considerations." Pharmaceutical Technology 14 (October 1990): 54-60.

Pfister, William R. "Silicone Adhesives for Transdermal Drug Delivery Systems." Drug and Cosmetic Industry (October 1988): 44-52.

Pfister, William R. and Dean S. Hsieh. "Permeation Enhancers Compatible with Transdermal Drug Delivery Systems. II: System Design Considerations." Pharmaceutical Technology 14 (October 1990): 54-60.

Pieta, Piergiogio, Angelo Rava and Pierantonio Biondi. "High-Performance Liquid Chromatography of Nifedipine, its Metabolites and Photochemical Degradation Products." Joumal of Chromatography 210 (1981): 516-21.

Potts Russell O., Vivien H.W. Mak, Richard H. Guy and Michael L. Francoeur. "Strategies to Enhance Permeability via Stratum Corneum Lipid Pathways." Advanced in Lipid Research 24 (1991): 173-210.

Potts, Russell O. and Richard H. Guy. "Predicting Skin Permeability." 
Pharmaceutical Research 9 (May 1992): 663-669.

Rasool, Ala Abdul, Anwar A. Hussain, and Lewis W. Dittert. "Solubility Enhancement of Some Water-Insoluble Drugs in the Presence of Nicotinamide and Related Compounds." Joumal of Pharmaceutical Sciences 80 (April, 1991): 387-93.

Reichek, N. Transdermal delivery of nitroglycerin I. In Yie W. Chien, Editor. Transdermal Controlled Systemic Medications. Marcel Dekker, NY 1987, 227-244.

Robinson, Dennis and John W. Mauger. "Drug Delivery Systems" American Joumal of Hospital Pharmacy 48 (October 1991): S14-S23 .

Rolfe D. "Chemical and Physical Methods of Enhancing Transdermal Drug Delivery." Pharmaceutical Technology (September, 1988): 130-140.

Roy, Samir D. and Gordon L. Flynn. "Solubility Behavior of Narcotic Analgesics in Aqueous Media: Solubilities and Dissociation Constants of Morphine, Fentanyl and Surfentanil." Pharmaceutical Research 6 (February 1989): 147-151.

Ruddy, Stephen B. and Boka W. Hadzija. Influence of Molecular Size on the Iontophoretic Transport of Polymeric Nonelectrolytes Through Hairless Rat Skin. Paper presented at the GRASP Eastern Regional Meeting, Durham, N.C. 23 May 1991.

Sarpot P.S. and Joel L. Zatz. "Percutaneous Absorption Enhancement by Nonionic Surfactants. " Drug Development in Industrial Pharmacy, 13 (1987): 15-37.

Satas, Donatas, ed. Handbook of Pressure Sensitive Adhesive Technology, 2nd ed. New York: Van Nostrand Reinhold 1989.

Saxena, N.K. and A.K. Nigram. "Restricted Exploration of Mixtures by Symmetric Simplex Designs." Technometrics 19 (February 1977): 47-52.

Scheuplein, Robert J. "Physiology of the Skin" In Alfred Jarrett, ed. The Physiology and Pathophysiology of the Skin. New York: Academic Press, 1978.

Scheuplein, Robert J. and Lisa Ross. "Effects of Surfactants and Solvents on the Permeability of Epidermis. "Journal of the Society of Cosmetic Chemists 21 (December 1970): 853-74.

Schwartz Janice B., Robert A. Upton, Emil T. Lin, Roger L. Williams, and Lezlie Z. Benet. "Effect of Cimetidine or Ranitidine Administration on Nifedipine Pharmacokinetics and Pharmacodynamics." Clinical Pharmacology and Therapeutics 43 (June 1988): 673-80. 
Seki, T., K. Sugibayashi, and Y. Morimoto. "Effect of Solvents on the Permeation of Nicardipine Hydrochloride though Hairless Rat Skin." Chemical Pharmaceutical Bulletin 35 (1987): 3054-7.

Shapiro, Samuel S. and John A. Comell. The ASQC Basic References in Quality Control: Statistical Techniques, Volume 5: How to Run Mixture Experiments for Product Quality. Milwaukee, Wisconsin 1983.

Sheretz E.F., Kenneth B. Sloan, R.G. McTieman. "Transdermal Delivery of 5-Fluorouracil Through Skin of Hairless Mice and Humans In Vitro: a Comparison of the Effect of Formulations and a Prodrug." Archives of Dermatological Research 282 (July 1990): 463-8.

Sheth, N.V., Freeman, D.J., Higuchi, W.I. and Spruance, S.L. "The Influence of Azone, Propylene Glycol and Polyethylene Glycol on In Vitro Skin Penetration of Triflurothymidine." International Journal Pharmaceutics 28 (1986): 201-9.

Siddiqui, Ovais. "Physicochemical, Physiological, and Mathematical Considerations in Optimizing Percutaneous Absorption of Drugs." Critical Reviews in Therapeutic Drug Carrier Systems 6 (January 1989): 1-38.

Sloan, Kenneth B, Kevin G. Siver, and Suzanne A.M. Koch. "The Effect of Vehicle on the Diffusion of Salicylic Acid Through Hairless Mouse Skin." Joumal of Pharmaceutical Sciences 75 (August, 1986): 744-49.

Sloan, Kenneth B., Suzanne A.M. Koch, Kevin G. Siver, and Franklin P. Flowers. "Use of Solubility Parameters of Drug and Vehicle to Predict Flux Through Skin." Journal of Investigative Dermatology 87 (1986): 244-52.

Sloan, Kenneth B., ed. "Use of Solubility Parameters from Regular Solution Theory to Describe Partitioning-Driven Processes." In Prodrugs New York: Marcel Dekker, Inc., 1992.

Smith, R.D., D.E. Robinson, B. Delighieres, B.D. Alberson, T.P. Tomal, M.J. Zinaman, and J.A. Simon. "Effects of Vehicle Supplementation on Total Estradiol Absomtion from a Transdermal Estradiol Delivery System. " Fertility and Sterility 56 (December 1991): 1029-33.

Snee, Ronald D. and D.W. Marquardt. "Extreme Vertices Designs for Linear Mixture Models." Technometrics 16 (1974): 399-408.

Snee, Ronald D. "Experimental designs for quadratic models in constrained mixture spaces." Technometrics 17 (1975): 517-28. 
Squillante, Emilio. "Physicochemical Parameters Affecting Percutaneous Absorption of Nifedipine." Ph.D. Dissertation, University of Rhode Island, 1993.

Stoughton, Richard B, and William O. McClure. "Azone: A new non-toxic enhancer of cutaneous penetration." Drug Development in Industrial Pharmacy 9 (April 1983): $725-44$.

Surber, Christian, Klaus P. Wilhelm, Mitshuhiko Hori, Howard I. Maibach, and Richard H. Guy. "Optimization of Topical Therapy: Partitioning of Drugs into Stratum Comeum." Pharmaceutical Research 7 (December, 1990): 1320-4.

Swanson, D.R., B.L. Barclay, P.S. Wong and F. Theeuwes. "Nifedipine Gastrointestinal Therapeutic System." American Journal of Medicine 83 (December 1987): 3-9.

Thomas, Stephen and William Pfister. "The Emerging Role of Silicones Used in Transdermal Drug Delivery Systems." Sciences Techniques Pharmatiques Sciences 1 (1991): 38-46.

Toddywala, Rohinton and Yie W. Chien, "Evaluation of Silicone Based PressureSensitive Adhesives for Transdermal Drug Delivery II. Effect of Penetrant Lipophilicity." Drug Development and Industrial Pharmacy 17 (February 1991): 24569.

Tojo, Kakuji and Ae-Ri C. Lee. "A Method for Predicting Steady-state Rate of Skin Penetration In Vivo." Journal of Investigative Dermatology, 92 (January 1989): 105-8.

Toshinobu, Seki. "Percutaneous Absorption Enhancer Applied to Membrane Permeation Controlled Transdermal Delivery of Nicardipine HCl." Drug Design and Delivery, 4 (1989): 69-75.

Turi, J.S. Danielson D. and J.W. Wotersom. "Effects of Polyoxypropylene 15Stearyl Ether and Propylene Glycol on Percutatneous Penetration Rate of Diflorasone Diacetate." Journal of Pharmaceutical Sciences 68 (March 1979): 275-80.

Twist, John N. and Joel L. Zatz, "A Model for Alcohol-Enhanced Permeation through Polymethylsiloxane Membranes. " Journal of Pharmaceutical Sciences 37 (January 1986): 429-44.

Twist, John N. and Joel L. Zatz, "A Model for Alcohol-enhanced Permeation Through Polydimethylsiloxane Membranes." Journal of Pharmaceutical Sciences 79 (January 1990): 28-31.

Valia, K.H., Yie W. Chien. "Long Term Skin Permeation Kinetics of Estradiol I. 
Effect of Drug Solubilizer-Polyethylene Glycol 400." Drug Development in Industrial Pharmacy 10 (1984) 951-3.

Vaughan, C.D. "Using Solubility Parameters in Cosmetic Formulation." Joumal of the Society of Cosmetic Chemists 36 (May 1985): 319-34.

Wiechers, Johann W. and Rokus A. De Zeeuw. "Transdermal Drug Delivery: Efficay and Potential Applications of the Penetration Enhancer Azone." Drug Design and Delivery 6 (June 1990): 87-100.

Wiechers, Johann W. "The Barrier Function of the Skin In Relation to Percutaneous Absorption of Drugs." Pharmaceutisch Weekblad-Scientific Edition 11 (June 1989): $185-98$.

Woodford, R. and B.W. Barry. "Optimization of Bioavailability of Topical Steroids: Thermodynamic Control." Joumal of Investigative Dermatology. 79 (June 1982): $388-91$.

Wotton, Paul K., Birgitte Mollgaard, Jonathan Hadgraft, and Annie Hoelgaard. "Vehicle Effect on Topical Drug Delivery. III. Effect of Azone on the Cutaneous Permeation of Metronidazole and Propylene Glycol." International Journal of Pharmaceutics, 24 (1985): 19-26.

Xu, Ping and Y.W. Chien. "Enhanced Skin Permeability for Transdermal Drug Delivery: Physiological and Physicochemical Considerations." Critical Reviews in Therapeutic Drug Carrier Systems, 8 (March 1991): 211-36.

Yum S.I. "Transdermal Therapeutic Systems and Rate Controlled Drug Delivery." Medical Program Technology, 15 (1989): 47-52. 